\title{
APPLICATION OF IN SITU AND EX SITU CHARACTERIZATION OF ATOMIC LAYER DEPOSITION PROCESSES FOR GALLIUM PHOSPHIDE AND SODIUM
}

FLUORIDE

by

Sara Rose Kuraitis

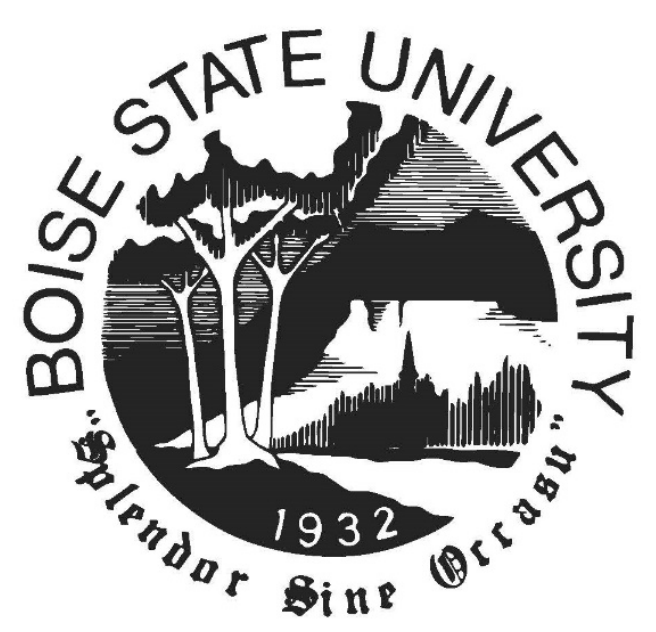

A dissertation

submitted in partial fulfillment

of the requirements for the degree of

Doctor of Philosophy in Materials Science and Engineering

Boise State University

August 2021 
(C) 2021

Sara Rose Kuraitis

\section{ALL RIGHTS RESERVED}




\title{
BOISE STATE UNIVERSITY GRADUATE COLLEGE
}

\section{DEFENSE COMMITTEE AND FINAL READING APPROVALS}

\author{
of the dissertation submitted by
}

Sara Rose Kuraitis

Dissertation Title: Application of in situ and ex situ Characterization of Atomic Layer Deposition Processes for Gallium Phosphide and Sodium Fluoride

Date of Final Oral Examination: $\quad 26$ March 2021

The following individuals read and discussed the dissertation submitted by student Sara Rose Kuraitis, and they evaluated their presentation and response to questions during the final oral examination. They found that the student passed the final oral examination.

Elton Graugnard, Ph.D. Chair, Supervisory Committee

Jeunghoon Lee, Ph.D. Member, Supervisory Committee

David Estrada, Ph.D. Member, Supervisory Committee

Jeffrey W. Elam, Ph.D. Member, Supervisory Committee

Steven Hues, Ph.D. Member, Supervisory Committee

The final reading approval of the dissertation was granted by Elton Graugnard, Ph.D., Chair of the Supervisory Committee. The dissertation was approved by the Graduate College. 


\section{DEDICATION}

I would like to dedicate my dissertation to my parents, who fostered my love of

learning from early on in life, and to my husband, who was by my side through the many years I spent as a perpetual student. 


\section{ACKNOWLEDGMENTS}

I would like to thank the many students, faculty members, and staff at Boise State University who helped throughout my time in the Materials Science \& Engineering department. Jake Soares worked with me on the re-design and construction of the custom ALD reactor and frequently helped with sample characterization. J.D. Hues fixed lots of broken equipment to keep the lab up and running and helped with XPS data collection. All the staff in the Boise State Surface Science Lab, the Boise State Center for Materials Characterization, and the Idaho Microfabrication Lab were invaluable in training me on equipment use and aiding with characterization and analysis. The Micron School of Materials Science \& Engineering and the Nanoscale Materials \& Device Group at Boise State University provided continuous support throughout my time with them. I owe my transition into Materials Science to Dr. William Hughes, who convinced me to completely change paths after I took an undergraduate course from him.

I would also like to thank Dr. Donghyeon Kang, Dr. Anil Mane, and Dr. Jeffrey Elam for hosting me and working extensively with me during my visit to Argonne National Laboratory.

Finally, I would like to thank my advisor Dr. Elton Graugnard and my committee members (Dr. Jeunghoon Lee, Dr. David Estrada, Dr. Jeffrey Elam, and Dr. Steven Hues) for their patience, support, and guidance in completing this research project. 


\begin{abstract}
Atomic layer deposition (ALD) is a vapor deposition technique for synthesizing thin films with nanometer thickness control. ALD films are deposited on a substrate surface in a cyclic layer-by-layer fashion utilizing alternating doses of highly reactive chemical precursors. Precursors are selected to undergo self-limiting chemical reactions with the surface, and desired film thickness is achieved by varying the number of ALD cycles accordingly. Optimization of ALD process parameters and precursor chemistry enables conformal coating of arbitrary substrate geometries, including high aspect ratio features such as trenches. In the decades since its introduction, ALD has been used for applications across many industries, including semiconductor device manufacturing, emerging battery technologies, and optoelectronics.

In this work, I present investigation of two previously reported chemistries for ALD of gallium phosphide (GaP), as well as improvements made to a custom ALD reactor to facilitate better process control and characterization. I also present a new process for thermal ALD of sodium fluoride $(\mathrm{NaF})$, with potential applications in electrode coatings for sodium-ion batteries. To my knowledge, this is the first report of NaF ALD. Finally, I summarize obstacles which may be addressed in future studies that build upon this work.
\end{abstract}




\section{TABLE OF CONTENTS}

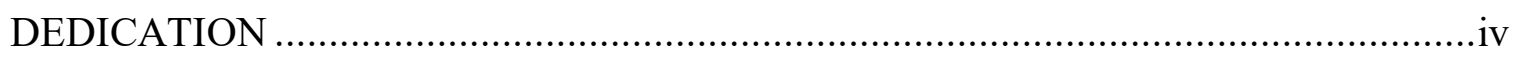

ACKNOWLEDGMENTS ............................................................................

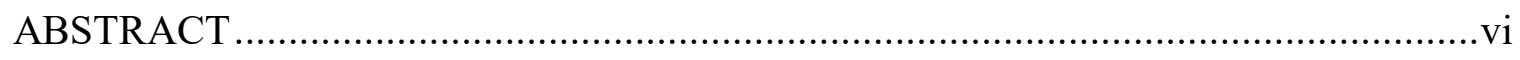

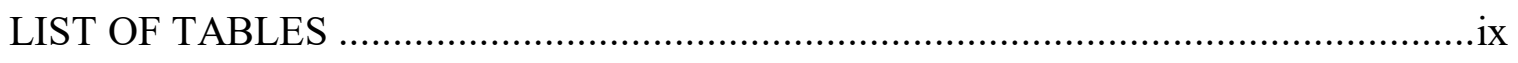

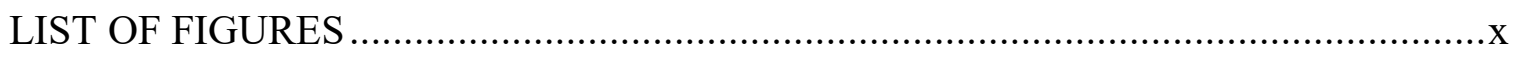

LIST OF ABBREVIATIONS ...................................................................

CHAPTER ONE: INTRODUCTION TO ATOMIC LAYER DEPOSITION.................. 1

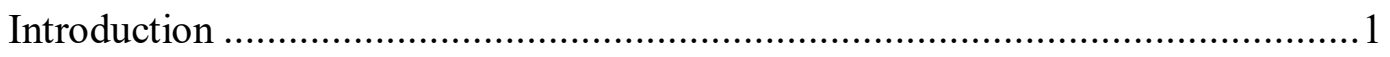

Fundamentals of the ALD Process ........................................................... 3

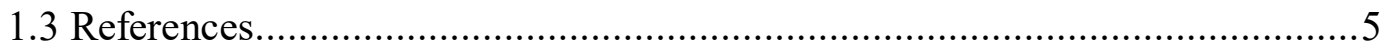

CHAPTER TWO: TOWARDS ATOMIC LAYER DEPOSITION OF GALLIUM

PHOSPHIDE THIN FILMS ............................................................................ 10

2.1 Introduction................................................................................ 10

2.2 ALD System Enhancements for Process Development ............................ 11

2.3 Characterization of GaP ALD: Experimental Methods ............................22

2.3.1 ALD of GaP ..............................................................22

2.3.2 Characterization...........................................................23

2.4 Characterization of GaP ALD: Results and Discussion .............................24

2.5 Summary and Conclusions ..........................................................45

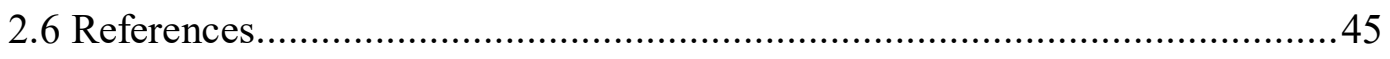




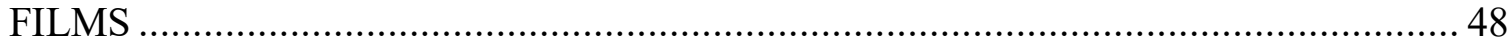

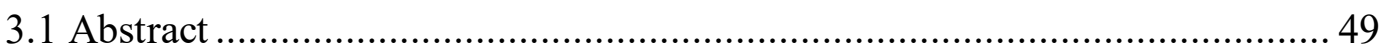

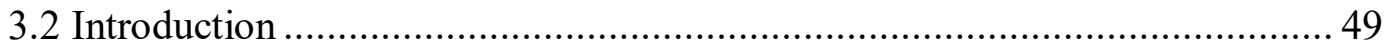

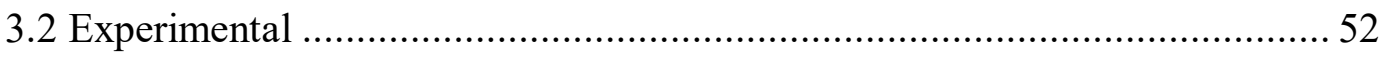

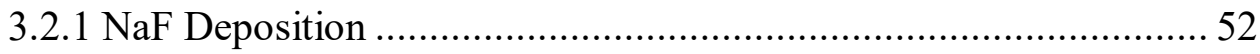

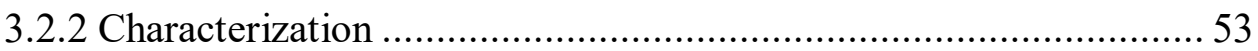

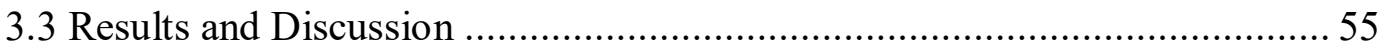

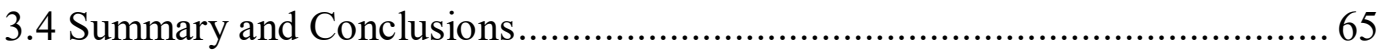

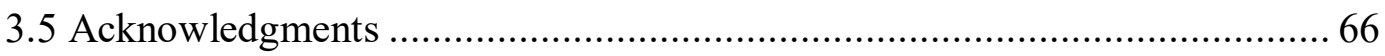

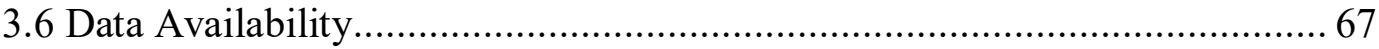

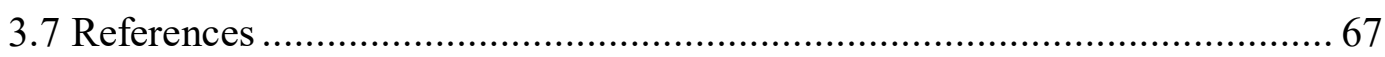

CHAPTER FOUR: CONCLUSIONS AND FUTURE DIRECTIONS....................... 70

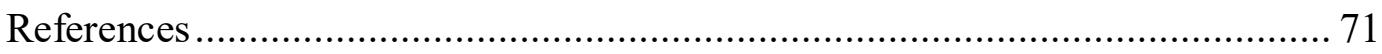

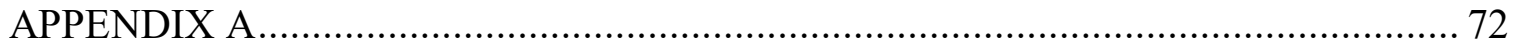

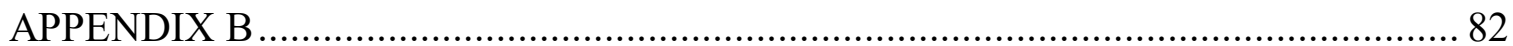




\section{LIST OF TABLES}

Table B1 $\quad \mathrm{NaO}^{t} \mathrm{Bu}+$ HF-pyridine dose and purge times for data shown in Figure B1. All time values are in seconds..............................................................83

Table B2 XPS Peak fitting results for NaF deposited with 3-20-2-15s cycle timing at various growth temperatures. 


\section{LIST OF FIGURES}

Figure 1.1 Schematic illustrating the hallmarks of ALD: film uniformity, conformality over large aspect-ratio features, and precision thickness control. Reproduced with permission from Handbook of Crystal Growth. ${ }^{28}$

Figure 1.2 Schematic of the binary ALD cycle for deposition of Al2O3 using trimethylaluminum and water (TMA + H2O).

Figure 1.3 Graphical representation of ALD process temperature dependence. Growth per cycle (GPC) is constant throughout the so-called "ALD Window" for ideal ALD behavior, though in reality many processes show some decrease in growth due to desorption or changes in the phase of the deposited film. Below the ALD window, the GPC can be lower due to insufficient activation energy and incomplete surface reactions, or it can be higher as the result of increased precursor condensation. Above the ALD window, GPC can again vary depending on whether precursor decomposition (higher GPC) or surface species desorption (lower GPC) has a significant effect. Figure adapted from George ${ }^{20}$ and Ritala et al. ${ }^{44} . .4$

Figure 2.1 Photograph of the custom tube furnace based ALD system following enhancements to precursor delivery system and process metrology and control.

Figure 2.2 Schematic of the custom ALD system components including (i) precursor manifolds and delivery system, (ii) tube furnace, (iii) sample entry/process metrology port, (iv) process gas abatement and pumping..................... 12

Figure 2.3 Screenshot of LabVIEW software for control and monitoring of ALD reactor operation. Regions highlighted in red boxes are a) ALD recipe and system status indicators, b) ALD reactor controls, c) ALD reactor schematic with valve status indicators, d) real-time display of measured process values, and e) measured and calculated process parameters graphed as a function of time. Enlarged views of highlighted regions b-e are shown during program operation in Figures 2.4, 2.6, and 2.7.......... 14 
Figure 2.4 In-operation screenshot of Initialization tab for manual control of the ALD reactor, highlighted in region $b$ ) of Figure 2.3. Default precursor names and needle valve settings are automatically populated based on initialization cluster arrays containing precursor configuration details (see

Appendix A.1) .................................................................. 15

Figure 2.5 Screenshot of user controls for creating customized ALD Recipe Tables in the LabVIEW program. In addition to standard ALD precursor cycles, recipes may include automated changes to temperature and carrier gas flow. All recipe step types also include the option to toggle a pneumatic valve for sampling of residual reaction gases using a mass spectrometer. Future modifications will include implementation of automated FTIR scans, which have already been included in the front-panel ALD Recipe controls.

Figure 2.6 In-operation screenshot of valve status indicators and instantaneous process parameter values, highlighted in regions c-d) of Figure 2.3. The system is shown with all gas delivery lines diverted to exhaust and the chamber being held static, corresponding to the Hold recipe step in a FillHold-Purge ALD cycle. .18

Figure 2.7 In-operation screenshot of LabVIEW front-panel graphs on the G1 graphs tab, highlighted in region e) of Figure 2.3. The data shown were produced in Simulation Mode during program development. G2 and G3 tabs (not shown) contain graphs for $20 \mathrm{TCs}$ and all digital-output cDAQ lines, respectively.

Figure 2.8 Screenshot of user controls for setting safety limits, which prevent reactor operation if pressure, carrier gas flow, or temperature measurements fall outside acceptable ranges. Safety limits can be set to experiment-specific values as needed (i.e., higher temperature requirements for low vapor pressure precursors). Elements disabled in the setup file cluster arrays are automatically disabled and greyed out at run-time, as discussed in Appendix A.

Figure 2.9 High resolution SEM image of GaP film grown on $\mathrm{SiO}_{2} / \mathrm{Si}(100)$ at $450{ }^{\circ} \mathrm{C}$ using 150 cycles of TMGa + TDMAP. .25

Figure 2.10 AFM topography of GaP film grown on $\mathrm{SiO}_{2} / \mathrm{Si}(100)$ at $450{ }^{\circ} \mathrm{C}$ using 150 cycles of TMGa + TDMAP. RMS surface roughness was $\sim 0.85 \mathrm{~nm}$......26

Figure 2.11 $\mathrm{SEM}$ image of $\mathrm{GaP}$ film grown on $\mathrm{SiO}_{2} / \mathrm{Si}(100)$ at $450{ }^{\circ} \mathrm{C}$ using 150 cycles of TMGa + TDMAP. EDS data for this sample are provided in Figure 2.12 . .26 
Figure 2.12 EDS spectra of ALD GaP a) film and b) 3D rod-like structure grown on $\mathrm{SiO}_{2} / \mathrm{Si}(100)$ at $450{ }^{\circ} \mathrm{C}$ using 150 cycles of TMGa + TDMAP. Inset electron images show corresponding regions of the sample pictured in Figure 2.11 scanned for EDS area spectra.

Figure 2.13 SEM image of $\mathrm{GaP}$ film grown on $\mathrm{SiO}_{2} / \mathrm{Si}(100)$ at $450{ }^{\circ} \mathrm{C}$ using 150 cycles of TMGa + TBP. EDS data for this sample are provided in Figure 2.14

Figure 2.14 EDS spectra of ALD GaP a) film and b) 3D rod-like structure grown on $\mathrm{SiO}_{2} / \mathrm{Si}(100)$ at $450{ }^{\circ} \mathrm{C}$ using 150 cycles of $\mathrm{TMGa}+\mathrm{TBP}$. Inset electron images show corresponding regions of the sample pictured in Figure 2.13 scanned for EDS area spectra.

Figure 2.15 XPS of GaP film grown on $\mathrm{SiO}_{2} / \mathrm{Si}(100)$ at $450{ }^{\circ} \mathrm{C}$ using 150 cycles of $\mathrm{TMGa}+\mathrm{TBP}$.

Figure 2.16 XRD scan of $\mathrm{GaP}$ film grown on $\mathrm{SiO}_{2} / \mathrm{Si}(100)$ at $450{ }^{\circ} \mathrm{C}$ using 150 cycles of $\mathrm{TMGa}+\mathrm{TBP}$. Presence of GaP (111) and GaP (220) peaks indicates films were polycrystalline GaP. Red hash marks shown for $\mathrm{GaP}$ reflections from ICDD PDF 00-032-0397.

Figure 2.17 SEM image of $\mathrm{GaP}$ film grown on $\mathrm{SiO}_{2} / \mathrm{Si}(100)$ at $475^{\circ} \mathrm{C}$ using 150 cycles of TMGa + TDMAP, showing an increased number of rod-like 3D structures on top of the surface film. A high-resolution image (50X increase in magnification) of the center of this region is provided in Figure 2.18, and a cross-sectional image of the sample is provided in Figure 2.19.

Figure 2.18 SEM image of $\mathrm{GaP}$ film grown on $\mathrm{SiO}_{2} / \mathrm{Si}(100)$ at $475^{\circ} \mathrm{C}$ using 150 cycles of TMGa + TDMAP, showing detail view of 3D GaP structures (50X increase in magnification relative to Figure 2.17).

Figure 2.19 Cross-sectional SEM image showing GaP film and a large 3D GaP structure. The sample shown in Figures 2.17 and 2.18 (GaP deposited on $\mathrm{SiO}_{2} / \mathrm{Si}(100)$ at $475^{\circ} \mathrm{C}$ using 150 cycles of TMGa + TDMAP) was scored with a diamond scribe and cleaved prior to cross-sectional imaging. .......35

Figure 2.20 SEM image of GaP film grown on $\mathrm{SiO}_{2} / \mathrm{Si}(100)$ at $450{ }^{\circ} \mathrm{C}$ using 150 cycles of TMGa $+\mathrm{TBP}$, yielding a many small rod-like GaP structures scattered across the film surface. $\mathrm{SiO}_{2} / \mathrm{Si}(100)$ sample was cleaned in fuming $\mathrm{H}_{2} \mathrm{SO}_{4}$ and then stored in a desiccator for several weeks prior to the deposition experiment, suggesting the need for careful sample preparation with thorough cleaning immediately prior to $\mathrm{GaP}$ deposition. 
Figure 2.21 $\mathrm{SEM}$ image of $\mathrm{GaP}$ film grown on $\mathrm{SiO}_{2} / \mathrm{Si}(100)$ at $450{ }^{\circ} \mathrm{C}$ using 150 cycles of TMGa $+\mathrm{TBP}$, yielding a spherical agglomeration of rod-like $\mathrm{GaP}$ structures. $\mathrm{SiO}_{2} / \mathrm{Si}(100)$ sample was cleaned in fuming $\mathrm{H}_{2} \mathrm{SO}_{4}$ and then stored in a desiccator for several weeks prior to the deposition experiment, suggesting the need for careful sample preparation with thorough cleaning immediately prior to GaP deposition.

Figure 2.22 $\mathrm{SEM}$ image of $\mathrm{GaP}$ film grown on $\mathrm{SiO}_{2} / \mathrm{Si}(100)$ at $450{ }^{\circ} \mathrm{C}$ using 150 cycles of TMGa + TBP, yielding several spherical agglomerations of rodlike $\mathrm{GaP}$ structures atop a carpet-like coating of $\mathrm{GaP}$ rods. $\mathrm{SiO}_{2} / \mathrm{Si}(100)$ sample was cleaned in fuming $\mathrm{H}_{2} \mathrm{SO}_{4}$ and then stored in a desiccator for several weeks prior to the deposition experiment, suggesting the need for careful sample preparation with thorough cleaning immediately prior to GaP deposition.

Figure 2.23 QCM data for 50 full-flow ALD cycles of TMGa + TDMAP at $450{ }^{\circ} \mathrm{C}$ with 1-30-1-60s cycle timing using a $\mathrm{GaPO}_{4}$ resonator that was pre-coated with $\mathrm{ALD} \mathrm{SiO}_{2}$ at $150{ }^{\circ} \mathrm{C}$. The top graph shows the mass gain per unit area plotted as a function of experiment time, with precursor dosing status shown below. The bottom graph shows the calculated half-cycle mass gain for TMGa (blue) and TDMAP (orange).

Figure 3.1 Illustration of the ALD process for $\mathrm{NaF}$ using $\mathrm{NaO}^{t} \mathrm{Bu}$ and $\mathrm{HF}$-pyridine solution. Pyridine is not included in the schematic because it does not participate in the ALD surface reactions. The ALD cycle consists of four steps, each defined by a corresponding step time $t_{\mathrm{i}}$ : 1) $\mathrm{NaO}^{t} \mathrm{Bu}$ dose, 2) purge, 3) HF-pyridine dose, 4) purge. Temporal separation of the precursor doses ensures that reactions occur only at the substrate surface (not in the vapor phase), and ultimate film thickness is therefore determined by the number of $\mathrm{NaO}^{t} \mathrm{Bu}+\mathrm{HF}$-pyridine cycles completed. .52

Figure 3.2 QCM data for (a) steady-state NaF growth with 3-20-2-15 cycle timing, (b) detailed view of two cycles with 3-20-2-15 cycle timing, and (c) detailed view of two cycles with 3-60-2-20 cycle timing. The $x-$ and $y-$ scale values have been shifted so that both start at zero for the graphed data, though additional $\mathrm{NaF}$ cycles were completed prior those shown (in the case of Fig. 3.2a, 39 cycles were completed prior to the steady-state cycles shown). All QCM measurements were performed at $200{ }^{\circ} \mathrm{C}$ and normalized to QCM of alumina (trimethylaluminum + water) to correct for any back-side deposition on the QCM crystal. ....................................56

Figure 3.3 XPS data and analysis results for $\mathrm{NaF}$ film on silicon substrates. Deposition was performed at $200{ }^{\circ} \mathrm{C}$ with 100 ALD cycles of $\mathrm{NaO}^{t} \mathrm{Bu}+$ HF-pyridine using 3-20-2-15 cycle timing. (a) Survey scan, (b) Sodium 1s region, (c) Fluorine 1s region. 
Figure 3.4 Temperature dependence of NaF GPC, based on SE fitting results for 100 ALD cycles of $\mathrm{NaO}^{t} \mathrm{Bu}+\mathrm{HF}$-pyridine on silicon substrates. Duplicate samples from different regions of the reactor chamber (separated by $\sim 50$ $\mathrm{cm})$ are shown for each growth temperature. Calculated errors of the fitted thickness values are within the data markers. Films deposited at $250{ }^{\circ} \mathrm{C}$ (red) deviated slightly from the bulk NaF Sellmeier SE model, as shown in Appendix B.3 Fig. B13c-d.

Figure 3.5 Grazing incidence X-ray diffraction (GIXRD) data for NaF films deposited on silicon substrates with 100 ALD cycles of $\mathrm{NaO}^{t} \mathrm{Bu}+\mathrm{HF}-$ pyridine. Data are shown for films grown at $175,200,225$, and $250{ }^{\circ} \mathrm{C}$, and red ticks indicate the predicted reflections for cubic $\mathrm{NaF}$.

Figure 3.6 Argon-filled glovebox AFM images of NaF surface morphology for asdeposited films on silicon substrates at growth temperatures of (a) $175^{\circ} \mathrm{C}$, (b) $200^{\circ} \mathrm{C}$, (c) $225^{\circ} \mathrm{C}$, and (d) $250^{\circ} \mathrm{C}$. (e) Surface roughness $\mathrm{R}_{\mathrm{q}}$ values for various growth temperatures (blue $=$ as-deposited, orange $=$ airexposed). See Appendix B.4 for AFM images of air-exposed samples.... 65

Figure A1 Screenshot of Precursor Settings cluster array global variable used for initialization of chemical precursors. Settings configured in this array are utilized throughout the rest of the LabVIEW program.

Figure A2 Screenshot of VI block diagram section showing Producer/Consumer programming architecture used to continuously collect, process, and log ALD reactor data.

Figure A3 Screenshot of VI block diagram section with parallel loops to update front-panel graphs and indicators (Loop 3), respond to user input for information buttons and safety limits (Loop 4), and respond to user input for manual system operation and ALD recipe execution (Loop 5). ......... 79

Figure A4 Screenshot of first 7 lines of the Recipe Execution Array corresponding to the ALD Recipe shown in Figure 2.5. The Recipe Execution Array is stored as a global variable and accessed during recipe modification, recipe execution, and file writing. Each cluster array element contains all information needed for execution of a single recipe step......................... 80

Figure A5 Screenshot of ExecuteRecipeStep subVI block diagram for the case of a Fill-Hold-Purge recipe step. 
Figure B1 (a) Steady-state NaF growth with 3-20-2-15 cycle timing, reproduced from Fig. 3.2a. (b-f) NaF growth with various precursor dose and purge times provided in Table B1. Net mass change (black diamonds) for complete $\mathrm{NaO}^{t} \mathrm{Bu}+\mathrm{HF}$-pyridine cycle remained near $40-50 \mathrm{ng} / \mathrm{cm}^{2}$ for all dose and purge times shown. The blue and orange bars in the lower traces for each figure represent the mass changes from the individual $\mathrm{NaO}^{t} \mathrm{Bu}$ and HF-pyridine exposures, respectively. The $\mathrm{x}$ - and $\mathrm{y}$-scale values have been shifted so that both start at zero for the graphed data, though additional $\mathrm{NaF}$ cycles were completed prior those shown (in the case of Fig. B1a, 39 cycles were completed prior to the steady-state cycles shown). All QCM measurements were performed at $200^{\circ} \mathrm{C}$ and normalized to QCM of alumina (trimethylaluminum + water) to correct for any back-side deposition on the QCM crystal.

Figure B2 Average mass change values for various cycle timing combinations. Similar dosing parameters are grouped together along the horizontal axis.

Figure $\mathrm{B} 3 \quad$ (a) Repeated $\mathrm{NaO}^{t} \mathrm{Bu}$ doses with $0.5-10$ half-cycle timing. (b) Repeated $\mathrm{NaO}^{t} \mathrm{Bu}$ dosing with 3-60 half-cycle timing. Under the conditions shown in (b), the surface reactions do not self-limit and subsequent doses are non-saturating. All QCM measurements were performed at $200{ }^{\circ} \mathrm{C}$ and normalized to QCM of alumina (trimethylaluminum + water) to correct for any back-side deposition on the QCM crystal. 86

Figure B4 XPS surface spectra for $\mathrm{NaF}$ film deposited at $175^{\circ} \mathrm{C}$ with 3-60-2-15 cycle timing. Films were grown on silicon substrates with native oxide. (a) Survey scan, (b) Sodium 1s region, (c) Fluorine 1s region, (d) Oxygen 1s region with overlap of sodium KLL Auger peaks, (e) Carbon 1s region. For reference, the prominent Na KLL peak from panel (d) is marked with a black diamond in panel (a). .88

Figure B6 XPS surface spectra for $\mathrm{NaF}$ film deposited at $225^{\circ} \mathrm{C}$ with 3-60-2-15 cycle timing. Films were grown on silicon substrates with native oxide. (a) Survey scan, (b) Sodium 1s region, (c) Fluorine 1s region, (d) Oxygen 1s region with overlap of sodium KLL Auger peaks, (e) Carbon 1s region. For reference, the prominent Na KLL peak from panel (d) is marked with a black diamond in panel (a) .............................................................90

Figure B7 XPS surface spectra for $\mathrm{NaF}$ film deposited at $250{ }^{\circ} \mathrm{C}$ with $3-60-2-15$ cycle timing. Films were grown on silicon substrates with native oxide. (a) Survey scan, (b) Sodium 1s region, (c) Fluorine 1s region, (d) Oxygen 1s region with overlap of sodium KLL Auger peaks, (e) Carbon 1s region. For reference, the prominent Na KLL peak from panel (d) is marked with a black diamond in panel (a). 
Figure B8 XPS surface spectra for $\mathrm{NaF}$ film deposited at $200^{\circ} \mathrm{C}$ with 5-30-1-30 cycle timing. Films were grown on silicon substrates with native oxide. (a) Survey scan, (b) Sodium 1s region, (c) Fluorine 1s region, (d) Oxygen 1s region with overlap of sodium KLL Auger peaks, (e) Carbon 1s region. For reference, the prominent Na KLL peak from panel (d) is marked with a black diamond in panel (a)............................................................. 92

Figure B9 XPS surface spectra for $\mathrm{NaF}$ film deposited at $200^{\circ} \mathrm{C}$ with $3-60-2-15$ cycle timing. Films were grown on silicon substrates with native oxide. (a) Survey scan, (b) Sodium 1s region, (c) Fluorine 1s region, (d) Oxygen 1s region with overlap of sodium KLL Auger peaks, (e) Carbon 1s region. For reference, the prominent Na KLL peak from panel (d) is marked with a black diamond in panel (a).

Figure B10 CompleteEASE layers used to model $\mathrm{NaF}$ film on Si. Substrate $=$ silicon ("SI_JAW"), Layer \#1 = native oxide ("NTVE_JAW"), Layer \#2 = NaF ("NaF (Sellmeier)"). The Layer \#2 Sellmeier parameters "Einf," "UV Pole Amp.," "UV Pole En.," and "IR Pole Amp." correspond to Equation B1 variables $\varepsilon(\infty), A, B$, and $E$, respectively. 95

Figure B11 CompleteEASE layers used to model $\mathrm{NaF}$ film deposited at $200{ }^{\circ} \mathrm{C}$ on $\mathrm{Al}_{2} \mathrm{O}_{3}$ coated $\mathrm{Si}$. Substrate = silicon ("SI_JAW"), Layer \#1 = native oxide ("NTVE_JAW"), Layer \#2 = ALD $\mathrm{Al}_{2} \mathrm{O}_{3}$, Layer $\# 3=\mathrm{NaF}$ ("NaF (Sellmeier)"). The surface roughness value measured from AFM was included. The Layer \#3 Sellmeier parameters "Einf," "UV Pole Amp.," "UV Pole En.," and "IR Pole Amp." correspond to Equation B1 variables $\varepsilon(\infty), A, B$, and $E$, respectively.

Figure B12 SE data and modelling results for NaF films deposited via 100 ALD cycles of $\mathrm{NaO}^{t} \mathrm{Bu}+\mathrm{HF}-$ pyridine at (a-b) $175^{\circ} \mathrm{C}$ and (c-d) $200^{\circ} \mathrm{C}$ with 3-20-215 cycle timing. Duplicate samples for each temperature were taken from opposite ends of the reactor chamber (separated by $\sim 50 \mathrm{~cm}$ ).

Figure B13 SE data and modelling results for NaF films deposited via 100 ALD cycles of $\mathrm{NaO}^{t} \mathrm{Bu}+\mathrm{HF}-$ pyridine at (a-b) $225^{\circ} \mathrm{C}$ and (c-d) $250^{\circ} \mathrm{C}$ with $3-20-2-$ 15 cycle timing. Duplicate samples for each temperature were taken from opposite ends of the reactor chamber (separated by $\sim 50 \mathrm{~cm}$ ). 98

Figure B14 SE data and modelling results for NaF films deposited via 100 ALD cycles of $\mathrm{NaO}^{t} \mathrm{Bu}+\mathrm{HF}$-pyridine at $20{ }^{\circ} \mathrm{C}$ with (a-b) 5-30-1-30 and (c-d) 3-60$2-15$ cycle timing. Duplicate samples for each temperature were taken from opposite ends of the reactor chamber (separated by $\sim 50 \mathrm{~cm}$ ).

Figure B15 SE data and modelling results for $\mathrm{NaF}$ films deposited on $\mathrm{Al}_{2} \mathrm{O}_{3}$ coated silicon via $100 \mathrm{ALD}$ cycles of $\mathrm{NaO}^{t} \mathrm{Bu}+\mathrm{HF}$-pyridine at $200{ }^{\circ} \mathrm{C}$ with $3-$ 20-2-15 cycle timing. 100 
Figure B16 AFM images of NaF surface morphology for air-exposed films deposited on silicon substrates at growth temperatures of (a) $175^{\circ} \mathrm{C}$, (b) $200^{\circ} \mathrm{C}$, (c)

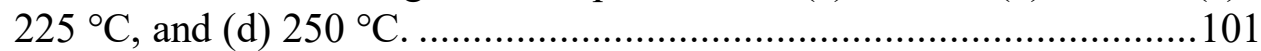

Figure B17 SEM micrographs of NaF surface morphology for air-exposed films deposited on silicon substrates at growth temperatures of (a) $175^{\circ} \mathrm{C}$, (b)

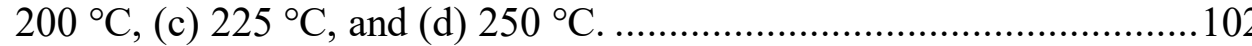




\section{LIST OF ABBREVIATIONS}

\begin{tabular}{|c|c|}
\hline AFM & Atomic Force Microscopy \\
\hline ALD & Atomic Layer Deposition \\
\hline cDAQ & Compact Data Acquisition System \\
\hline CEI & Cathode-Electrolyte Interface \\
\hline CVD & Chemical Vapor Deposition \\
\hline EDS & Energy-dispersive X-ray Spectroscopy \\
\hline FESEM & Field Emission Scanning Electron Microscope \\
\hline FF & Full-Flow \\
\hline FFD & Full-Flow with Diversion \\
\hline FHP & Fill-Hold-Purge \\
\hline FTIR & Fourier-Transform Infrared Spectroscopy \\
\hline $\mathrm{GaP}$ & Gallium Phosphide \\
\hline $\mathrm{GaPO}_{4}$ & Gallium Phosphate \\
\hline GIXRD & Grazing Incidence X-ray Diffraction \\
\hline GPC & Growth Per Cycle \\
\hline $\mathrm{HF}$ & Hydrofluoric acid \\
\hline $\mathrm{H}_{2} \mathrm{SO}_{4}$ & Sulfuric acid \\
\hline $\mathrm{L}$ & Langmuir \\
\hline LIB & Lithium-ion Battery \\
\hline MFC & Mass Flow Controller \\
\hline
\end{tabular}




\begin{tabular}{|c|c|}
\hline $\mathrm{NaF}$ & Sodium Fluoride \\
\hline $\mathrm{NaO}^{t} \mathrm{Bu}$ & Sodium tert-Butoxide \\
\hline NI & National Instruments \\
\hline PVD & Physical Vapor Deposition \\
\hline QCM & Quartz Crystal Microbalance \\
\hline RGA & Residual Gas Analysis \\
\hline RMS & Root Mean Square \\
\hline SE & Spectroscopic Ellipsometry \\
\hline SEI & Solid Electrolyte Interphase \\
\hline SEM & Scanning Electron Microscopy \\
\hline SIB & Sodium-ion Battery \\
\hline TBP & tert-Butylphosphine \\
\hline $\mathrm{TC}$ & Thermocouple \\
\hline TDMAP & Tris(dimethylamino)phosphine \\
\hline TEM & Transmission Electron Microscopy \\
\hline TEGa & Triethylgallium \\
\hline $\mathrm{TiCl}_{4}$ & Titanium tetrachloride \\
\hline TMA & Trimethylaluminum \\
\hline TMGa & Trimethylgallium \\
\hline TPS & Tris(tert-pentoxy)silanol \\
\hline VI & Virtual Instrument \\
\hline XPS & X-ray Photoelectron Spectroscopy \\
\hline XRD & X-ray Diffraction \\
\hline
\end{tabular}





\section{CHAPTER ONE: INTRODUCTION TO ATOMIC LAYER DEPOSITION}

\section{Introduction}

Thin film materials are at the forefront of many modern materials research and development efforts. With applications ranging from advanced memory storage, ${ }^{1-4}$ corrosion prevention, ${ }^{5-7}$ solar cells, ${ }^{8-12}$ rechargeable batteries,${ }^{13-17}$ flexible electronics,${ }^{18}$ and augmented reality devices, ${ }^{19}$ thin films have been engineered to impart the optical, electrical, and chemical properties needed for an ever-growing list of technological advancements.

There exist many techniques for depositing thin films including physical vapor deposition (PVD, e.g., sputtering, evaporation) and multiple varieties of chemical vapor deposition (CVD). For applications requiring nanometer-scale films with precise thickness control, atomic layer deposition (ALD) is ideally suited. A form of CVD, ALD is a bottom-up vapor deposition technique that employs cyclic alternating doses of highly reactive chemical precursors containing the constituent elements of the target film. ${ }^{20}$ The chemical precursors used in ALD exhibit self-limiting surface reactions, which gives ALD many advantages over other thin-film deposition techniques. ALD films are uniform and pinhole-free, with conformal growth over complex and large aspect-ratio geometries (Figure 1.1), such as microchannel plates and three-dimensional porous materials. ${ }^{21-26}$ Film thickness is controlled down to the atomic scale and can be fine-tuned by varying only the number of ALD cycle repetitions performed. Because ALD relies 
entirely on surface reactions, it is also inherently sensitive to substrate surface chemistry, which has been exploited to achieve area-selective ALD patterning. ${ }^{27}$

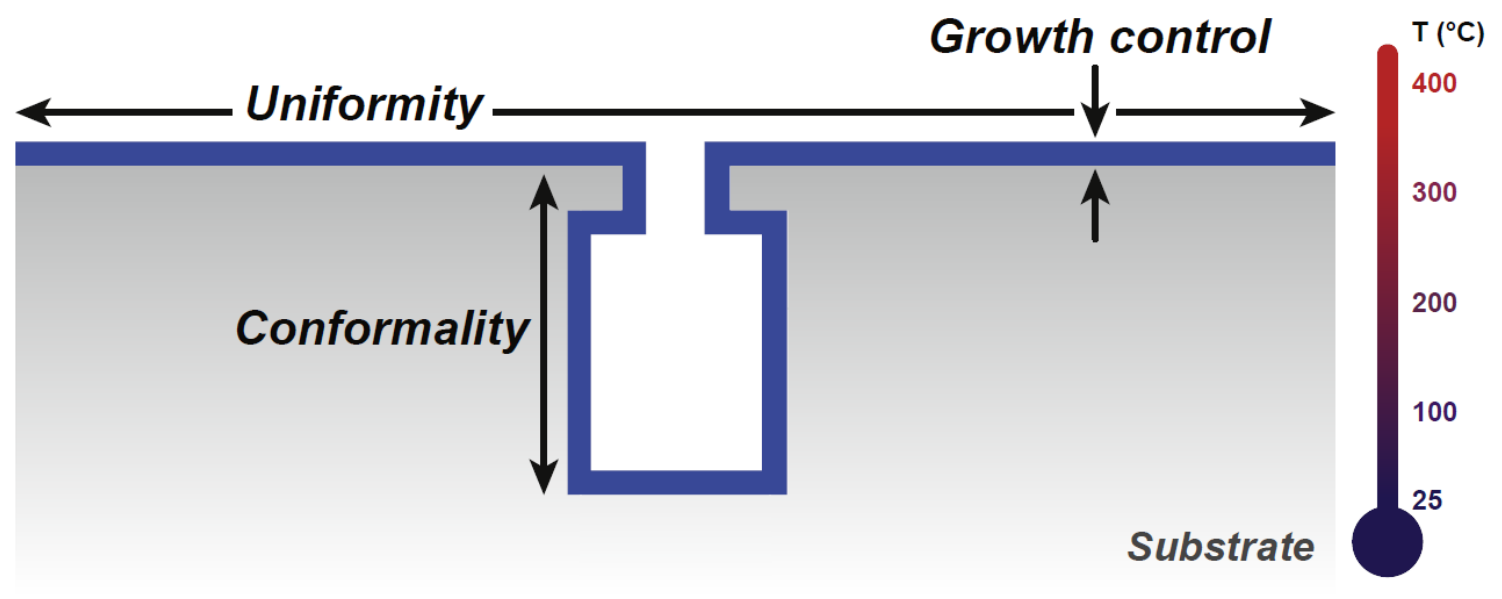

Figure 1.1 Schematic illustrating the hallmarks of ALD: film uniformity, conformality over large aspect-ratio features, and precision thickness control. Reproduced with permission from Handbook of Crystal Growth. ${ }^{28}$

In recent years, ALD research has expanded to a huge number of fields, and ALD processes have been developed for many applications ${ }^{29-31}$ including catalysis, ${ }^{32-36}$ surface passivation and corrosion protection, ${ }^{5,7} 3 \mathrm{D}$ memory storage and other semiconductor devices, ${ }^{1-4,37}$ photovoltaics, ${ }^{8,12}$ lithium-ion and next-generation batteries, ${ }^{13,16}$ coating of microchannel plates for high-sensitivity photodetectors, ${ }^{21-23}$ and biomedical devices. ${ }^{38,39}$ Although there now exist an enormous variety of materials that have been synthesized via ALD, ${ }^{40,41}$ development and characterization of novel chemistries for ALD remains an active field of research. ALD process development is non-trivial but is guided by a series of necessary and well-defined steps to gain insight into nucleation and growth of the deposited films as well as the factors influencing film properties. ${ }^{42}$ 


\section{Fundamentals of the ALD Process}

To understand the techniques and steps used in ALD process development, this section provides a brief overview of the ALD process. Figure 1.2 illustrates the ALD process for deposition of amorphous alumina (nominally $\mathrm{Al}_{2} \mathrm{O}_{3}$ ) using trimethylaluminum (TMA) and water, which has been extensively studied and serves as an informative example case. ${ }^{43}$ Substrates for film deposition are placed in a heated vacuum chamber and exposed to precursors one at a time. Following each precursor dose, the process chamber is purged with inert gas to remove excess precursor and reaction byproducts. Key process parameters include the pulse times and purge times for each precursor (shown as $t_{1}, t_{2}, t_{3}, t_{4}$ in Figure 1.2 for the binary chemistry process using trimethylaluminum and water), as well as substrate temperature, which defines the "ALD window" shown in Figure 1.3. Precursors are selected such that they are thermally stable at the target deposition temperature, which partly defines the bounds of the ALD window; thus, each step of the ALD process self-terminates once all surface reaction sites have been consumed, leading to the self-limiting reaction behavior and atomic-scale thickness control that is the hallmark of ALD. ${ }^{40}$ 


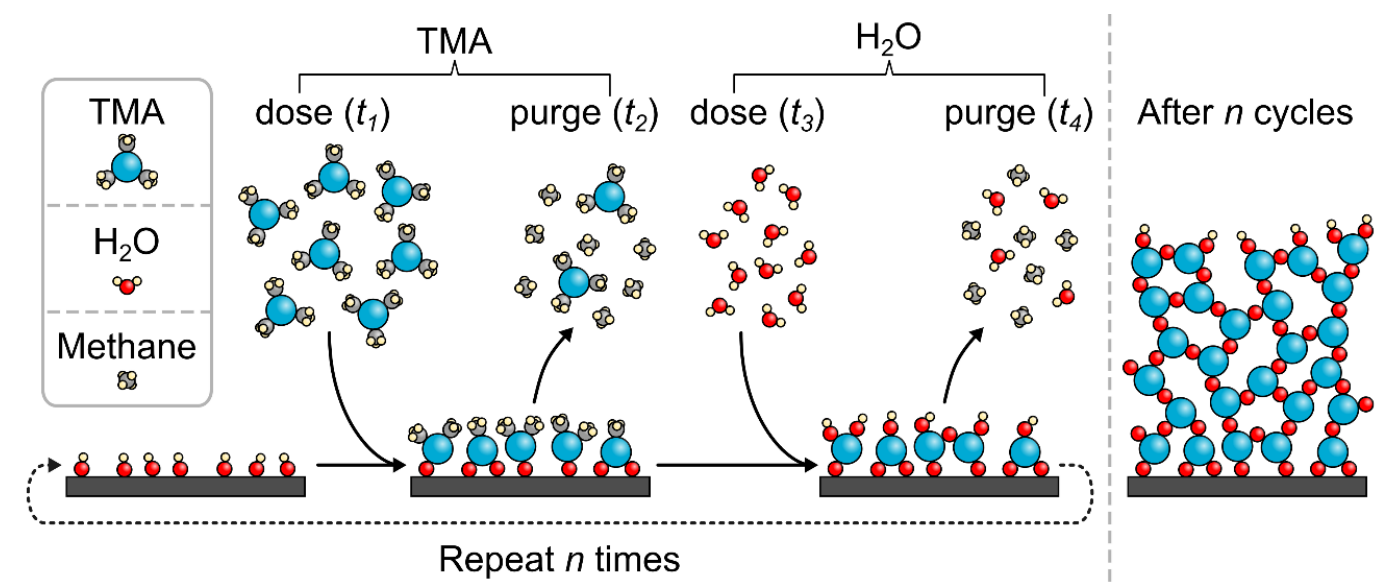

Figure 1.2 Schematic of the binary ALD cycle for deposition of Al2O3 using trimethylaluminum and water (TMA + H2O).

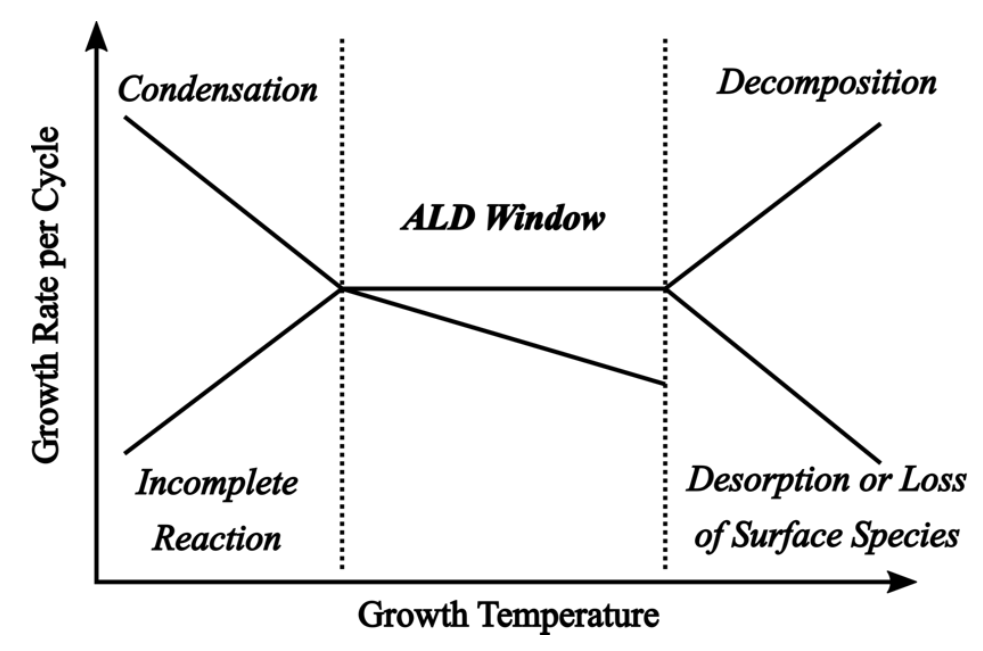

Figure 1.3 Graphical representation of ALD process temperature dependence. Growth per cycle (GPC) is constant throughout the so-called "ALD Window" for ideal ALD behavior, though in reality many processes show some decrease in growth due to desorption or changes in the phase of the deposited film. Below the

ALD window, the GPC can be lower due to insufficient activation energy and incomplete surface reactions, or it can be higher as the result of increased precursor condensation. Above the ALD window, GPC can again vary depending on whether precursor decomposition (higher GPC) or surface species desorption (lower GPC)

has a significant effect. Figure adapted from George ${ }^{20}$ and Ritala et al. ${ }^{44}$

High-purity chemical precursors can themselves be quite costly, and the repetition of many ALD cycles is inherently time consuming compared to other methods for thin- 
film deposition, such as CVD or sputtering. When the benefits of ALD's unique combination of conformal growth with nanometer thickness control outweigh the time and monetary costs, various approaches have been devised to mitigate these hurdles and allow for ALD to be scaled up for industrial-scale applications such as batch processing of powders and planar substrates for semiconductor device manufacturing. ${ }^{31}$

This body of work presents work on the development and characterization of ALD processes for two thin-film materials of interest - gallium phosphide (GaP) and sodium fluoride $(\mathrm{NaF})$. Chapter 2 presents work towards understanding the reaction mechanisms of two previously reported ALD chemistries for GaP films, which have many applications including photovoltaics and optoelectronics. Chapter 2 also includes the design and implementation of a modified thermal ALD reactor. Details are presented for a custom LabVIEW program used to control the ALD reactor and to synchronize in situ characterization and calculations with execution of the ALD recipe. Chapter 3 presents recently published work on a novel ALD chemistry for NaF films. No prior reports of NaF ALD have been published in the literature, so this newly developed ALD process represents a significant step towards $\mathrm{NaF}$ thin-film applications such as electrode coatings in sodium-ion batteries. Finally, Chapter 4 offers a roadmap for potential future directions building upon the foundation of this work.

\subsection{References}

1. Adinolfi, V.; Laudato, M.; Clarke, R.; Jewhurst, S.; McBriarty, M. E.; Hoang, S.; Narasimhan, V. K.; Cheng, L.; Littau, K. A., ALD Heterojunction Ovonic Threshold Switches. ACS Applied Electronic Materials 2020, 2 (12), 3818-3824.

2. Adinolfi, V.; Laudato, M.; Clarke, R.; Narasimhan, V. K.; Cheng, L.; Littau, K., Atomic layer deposition of germanium-selenium-tellurium compounds for lowleakage, tunable ovonic threshold switches. Journal of Vacuum Science \& Technology A 2020, 38 (5), 052404. 
3. Chen, Z.; Zhang, F.; Chen, B.; Zheng, Y.; Gao, B.; Liu, L.; Liu, X.; Kang, J., High-performance $\mathrm{HfOx} / \mathrm{AlOy}$-based resistive switching memory cross-point array fabricated by atomic layer deposition. Nanoscale Research Letters 2015, 10 (1), 70.

4. Yoo, C.; Kim, W.; Jeon, J. W.; Park, E.-S.; Ha, M.; Lee, Y. K.; Hwang, C. S., Atomic Layer Deposition of GexSe1-x Thin Films for Endurable Ovonic Threshold Selectors with a Low Threshold Voltage. ACS Applied Materials \& Interfaces 2020, 12 (20), 23110-23118.

5. Anderson, M. D.; Aitchison, B.; Johnson, D. C., Corrosion Resistance of Atomic Layer Deposition-Generated Amorphous Thin Films. ACS Applied Materials \& Interfaces 2016, 8 (44), 30644-30648.

6. Husain, E.; Narayanan, T. N.; Taha-Tijerina, J. J.; Vinod, S.; Vajtai, R.; Ajayan, P. M., Marine Corrosion Protective Coatings of Hexagonal Boron Nitride Thin Films on Stainless Steel. ACS Applied Materials \& Interfaces 2013, 5 (10), 4129-4135.

7. Li, M.; Jin, Z.-X.; Zhang, W.; Bai, Y.-H.; Cao, Y.-Q.; Li, W.-M.; Wu, D.; Li, A.-D., Comparison of chemical stability and corrosion resistance of group IV metal oxide films formed by thermal and plasma-enhanced atomic layer deposition. Scientific Reports 2019, 9 (1), 10438.

8. van Delft, J. A.; Garcia-Alonso, D.; Kessels, W. M. M., Atomic layer deposition for photovoltaics: applications and prospects for solar cell manufacturing. Semiconductor Science and Technology 2012, 27 (7), 074002.

9. Ali, N.; Hussain, A.; Ahmed, R.; Wang, M. K.; Zhao, C.; Haq, B. U.; Fu, Y. Q., Advances in nanostructured thin film materials for solar cell applications. Renewable and Sustainable Energy Reviews 2016, 59, 726-737.

10. Kanu, S. S.; Binions, R., Thin films for solar control applications. Proceedings of the Royal Society A: Mathematical, Physical and Engineering Sciences 2010, 466 (2113), 19-44.

11. Husain, A. A. F.; Hasan, W. Z. W.; Shafie, S.; Hamidon, M. N.; Pandey, S. S., A review of transparent solar photovoltaic technologies. Renewable and Sustainable Energy Reviews 2018, 94, 779-791.

12. Raiford, J. A.; Oyakhire, S. T.; Bent, S. F., Applications of atomic layer deposition and chemical vapor deposition for perovskite solar cells. Energy \& Environmental Science 2020, 13 (7), 1997-2023.

13. Pearse, A. J.; Schmitt, T. E.; Fuller, E. J.; El-Gabaly, F.; Lin, C.-F.; Gerasopoulos, K.; Kozen, A. C.; Talin, A. A.; Rubloff, G.; Gregorczyk, K. E., Nanoscale Solid State Batteries Enabled by Thermal Atomic Layer Deposition of a Lithium Polyphosphazene Solid State Electrolyte. Chemistry of Materials 2017, 29 (8), 3740-3753.

14. Mukanova, A.; Jetybayeva, A.; Myung, S.-T.; Kim, S.-S.; Bakenov, Z., A minireview on the development of Si-based thin film anodes for Li-ion batteries. Materials Today Energy 2018, 9, 49-66. 
15. Zhu, Y.; Gonzalez-Rosillo, J. C.; Balaish, M.; Hood, Z. D.; Kim, K. J.; Rupp, J. L. M., Lithium-film ceramics for solid-state lithionic devices. Nature Reviews Materials 2020.

16. Wang, X.; Yushin, G., Chemical vapor deposition and atomic layer deposition for advanced lithium ion batteries and supercapacitors. Energy \& Environmental Science 2015, 8 (7), 1889-1904.

17. Qi, Z.; Wang, H., Advanced Thin Film Cathodes for Lithium Ion Batteries. Research 2020, 2020, 24.

18. Datta, R. S.; Syed, N.; Zavabeti, A.; Jannat, A.; Mohiuddin, M.; Rokunuzzaman, M.; Yue Zhang, B.; Rahman, M. A.; Atkin, P.; Messalea, K. A.; Ghasemian, M. B.; Gaspera, E. D.; Bhattacharyya, S.; Fuhrer, M. S.; Russo, S. P.; McConville, C. F.; Esrafilzadeh, D.; Kalantar-Zadeh, K.; Daeneke, T., Flexible twodimensional indium tin oxide fabricated using a liquid metal printing technique. Nature Electronics 2020, 3 (1), 51-58.

19. Zhan, T.; Yin, K.; Xiong, J.; He, Z.; Wu, S.-T., Augmented Reality and Virtual Reality Displays: Perspectives and Challenges. iScience 2020, 23 (8), 101397.

20. George, S. M., Atomic Layer Deposition: An Overview. Chemical Reviews 2010, $110(1), 111-131$.

21. Cao, W.; Zhu, B.; Bai, X.; Xu, P.; Wang, B.; Qin, J.; Gou, Y.; Lei, F.; Liu, B.; Guo, J.; Zhu, J.; Bai, Y., High-Sensitivity and Long-Life Microchannel Plate Processed by Atomic Layer Deposition. Nanoscale Research Letters 2019, 14 (1), 153.

22. Mane, A. U.; Peng, Q.; Elam, J. W.; Bennis, D. C.; Craven, C. A.; Detarando, M. A.; Escolas, J. R.; Frisch, H. J.; Jokela, S. J.; McPhate, J.; Minot, M. J.; Siegmund, O. H. W.; Renaud, J. M.; Wagner, R. G.; Wetstein, M. J., An Atomic Layer Deposition Method to Fabricate Economical and Robust Large Area Microchannel Plates for Photodetectors. Physics Procedia 2012, 37, 722-732.

23. O'Mahony, A.; Craven, C. A.; Minot, M. J.; Popecki, M. A.; Renaud, J. M.; Bennis, D. C.; Bond, J. L.; Stochaj, M. E.; Foley, M. R.; Adams, B. W.; Mane, A. U.; Elam, J. W.; Ertley, C.; Siegmund, O. H. W., Atomic layer deposition of alternative glass microchannel plates. Journal of Vacuum Science \& Technology A 2015, 34 (1), $01 \mathrm{~A} 128$.

24. Elam, J. W., Coatings on High Aspect Ratio Structures. Atomic Layer Deposition of Nanostructured Materials 2012, 227-249.

25. Graugnard, E.; Chawla, V.; Lorang, D.; Summers, C. J., High filling fraction gallium phosphide inverse opals by atomic layer deposition. Applied Physics Letters 2006, 89 (21), 211102.

26. King, J. S.; Graugnard, E.; Summers, C. J., TiO 2 Inverse Opals Fabricated Using Low-Temperature Atomic Layer Deposition. Advanced Materials 2005, 17 (8), 1010 1013.

27. Parsons, G. N.; Clark, R. D., Area-Selective Deposition: Fundamentals, Applications, and Future Outlook. Chemistry of Materials 2020, 32 (12), 4920-4953. 
28. Knoops, H. C. M.; Potts, S. E.; Bol, A. A.; Kessels, W. M. M., 27 - Atomic Layer Deposition. In Handbook of Crystal Growth (Second Edition), Kuech, T. F., Ed. North-Holland: Boston, 2015; pp 1101-1134.

29. Johnson, R. W.; Hultqvist, A.; Bent, S. F., A brief review of atomic layer deposition: from fundamentals to applications. Materials Today 2014, 17 (5), 236-246.

30. Im, H.; Wittenberg, N. J.; Lindquist, N. C.; Oh, S.-H., Atomic layer deposition: A versatile technique for plasmonics and nanobiotechnology. Journal of Materials Research 2012, 27 (4), 663-671.

31. Ritala, M.; Niinistö, J., Industrial Applications of Atomic Layer Deposition. ECS Transactions 2019, 25 (8), 641-652.

32. Chen, Z.; Zhang, G.; Prakash, J.; Zheng, Y.; Sun, S., Rational Design of Novel Catalysts with Atomic Layer Deposition for the Reduction of Carbon Dioxide. Advanced Energy Materials 2019, 9 (37), 1900889.

33. Eswar, N. K. R.; Singh, S. A.; Heo, J., Atomic layer deposited photocatalysts: comprehensive review on viable fabrication routes and reactor design approaches for photo-mediated redox reactions. Journal of Materials Chemistry A 2019, 7 (30), 1770317734.

34. Hengwei Wang, J. L. U., Atomic Layer Deposition: A Gas Phase Route to Bottom-up Precise Synthesis of Heterogeneous Catalyst. Acta Physico-Chimica Sinica 2018, 34 (12), 1334-1357.

35. O’Neill, B. J.; Jackson, D. H. K.; Lee, J.; Canlas, C.; Stair, P. C.; Marshall, C. L.; Elam, J. W.; Kuech, T. F.; Dumesic, J. A.; Huber, G. W., Catalyst Design with Atomic Layer Deposition. ACS Catalysis 2015, 5 (3), 1804-1825.

36. Ramachandran, R. K.; Detavernier, C.; Dendooven, J., Atomic Layer Deposition for Catalysis. Nanotechnology in Catalysis 2017, 335-358.

37. Jaggernauth, A.; Mendes, J. C.; Silva, R. F., Atomic layer deposition of high- $\kappa$ layers on polycrystalline diamond for MOS devices: a review. Journal of Materials Chemistry C 2020, 8 (38), 13127-13153.

38. Skoog, S. A.; Elam, J. W.; Narayan, R. J., Atomic layer deposition: medical and biological applications. International Materials Reviews 2013, 58 (2), 113-129.

39. Wadullah, H. M.; Ajeel, S. A.; Abbass, M. K., Synthesis and Characterization of Nanocoatings Thin films by Atomic Layer Deposition for Medical Applications. IOP Conference Series: Materials Science and Engineering 2019, 518, 032057.

40. Miikkulainen, V.; Leskelä, M.; Ritala, M.; Puurunen, R. L., Crystallinity of inorganic films grown by atomic layer deposition: Overview and general trends. Journal of Applied Physics 2013, 113 (2), 021301.

41. Atomic Limits - Database of ALD Processes.

https://www.atomiclimits.com/alddatabase/.

42. Vos, M.; Mackus, A. J. M.; Kessels , E. W. M. M. Atomic Layer Deposition Process Development - 10 steps to successfully develop, optimize and characterize ALD 
recipes 2019. https://www.atomiclimits.com/2019/02/12/atomic-layer-depositionprocess-development-10-steps-to-successfully-develop-optimize-and-characterize-aldrecipes/.

43. Puurunen, R. L., Surface chemistry of atomic layer deposition: A case study for the trimethylaluminum/water process. Journal of Applied Physics 2005, 97 (12), 121301.

44. Ritala, M.; Leskelä, M., Chapter 2 - Atomic layer deposition. In Handbook of Thin Films, Singh Nalwa, H., Ed. Academic Press: Burlington, 2002; pp 103-159. 


\section{CHAPTER TWO: TOWARDS ATOMIC LAYER DEPOSITION OF GALLIUM PHOSPHIDE THIN FILMS}

\subsection{Introduction}

Gallium phosphide (GaP) is a III-V semiconductor with a high transparency and refractive index $(n>3)$ throughout the visible range. ${ }^{1}$ Combined with its indirect bandgap of $2.26 \mathrm{eV}$, this makes $\mathrm{GaP}$ an ideal material for applications in optoelectronics, photonics, and photovoltaics. $^{2-9}$

Although ALD processes have been developed and thoroughly characterized for GaP-containing materials utilizing phosphine $\left(\mathrm{PH}_{3}\right)$ gas ${ }^{10-19}$ or plasma, ${ }^{20,21}$ little detail has been published for alternative phosphorus sources, such as tris(dimethylamino)phosphine (TDMAP) ${ }^{2,3}$ or tert-butylphosphine (TBP). ${ }^{4,5}$ TDMAP and TBP are both liquids at room temperature, and while TDMAP is both air-sensitive and flammable, TBP is pyrophoric and must be handled under inert gas. Although TDMAP and TBP are both air-reactive and release toxic byproducts, they are both considered as lower toxicity alternatives to $\mathrm{PH}_{3}$ gas, ${ }^{22}$ which can be instantly fatal in high concentrations. In this chapter, investigation of GaP ALD processes utilizing trimethylgallium (TMGa, pyrophoric liquid) as the gallium source with either TDMAP or TBP as the phosphine source is presented. Before GaP ALD process data are presented, this chapter presents enhancements to a tube furnace based ALD system to enable reliable process development. 


\subsection{ALD System Enhancements for Process Development}

To facilitate ALD process development, extensive modifications were made to a custom ALD reactor. These modifications serve to enhance the ability to synchronize ALD process parameters with real-time data acquired using in situ process metrology tools, such as a quartz crystal microbalance (QCM). The new reactor design increased precursor capacity up to 9 precursors split between four delivery manifolds, with the possibility of straightforward expansion to meet future needs. The design also eliminated cold spots in precursor delivery lines, which previously caused intermittent problems with precursor condensation. A photograph of the system and a schematic of the components are shown in Figures 2.1 and 2.2, respectively.

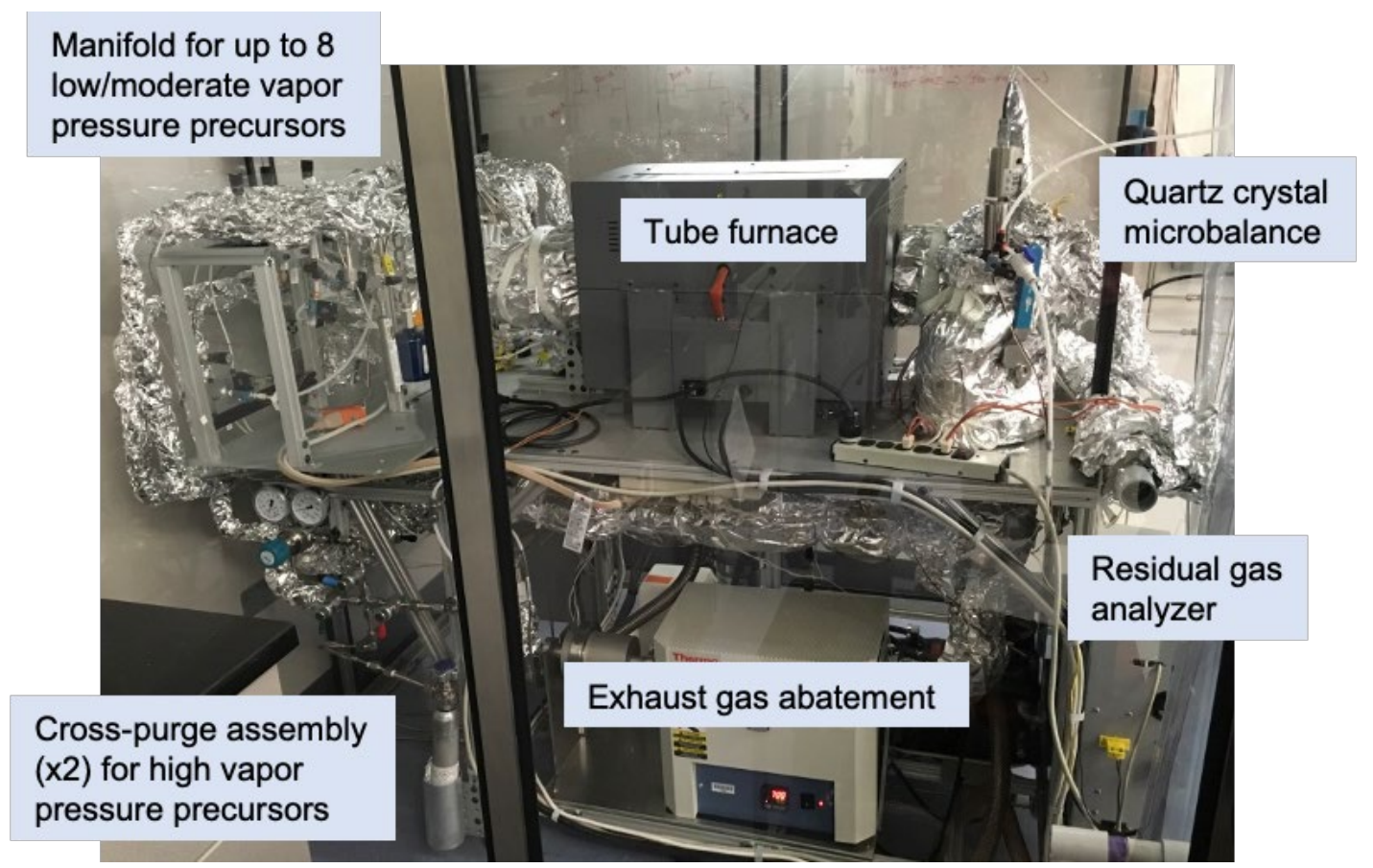

Figure 2.1 Photograph of the custom tube furnace based ALD system following enhancements to precursor delivery system and process metrology and control. 


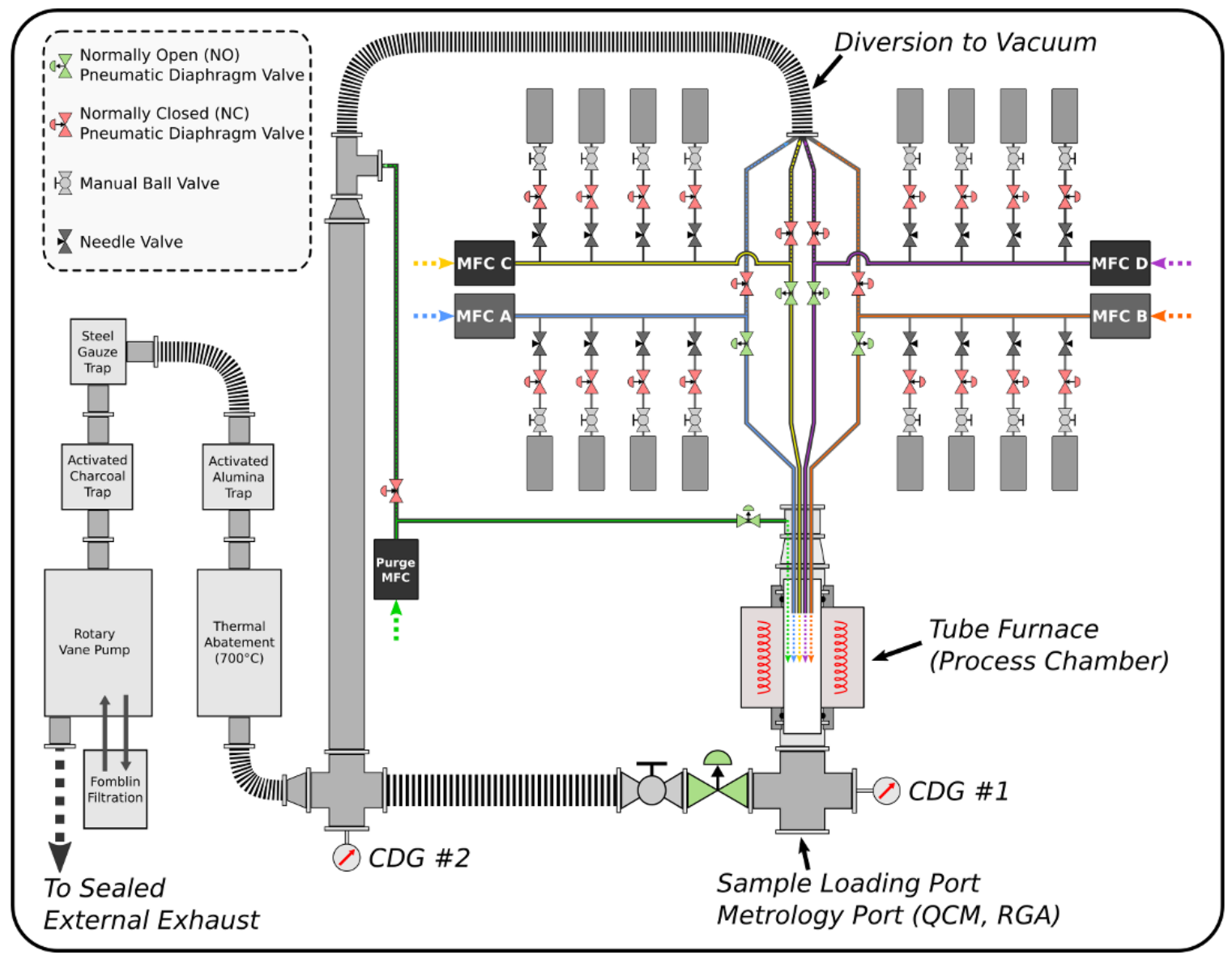

Figure 2.2 Schematic of the custom ALD system components including (i) precursor manifolds and delivery system, (ii) tube furnace, (iii) sample entry/process metrology port, (iv) process gas abatement and pumping.

A new control program or virtual instrument (VI) was created using National Instruments LabVIEW software to incorporate measurement and automatic logging of process parameters as well as execution of ALD recipe steps. This integration of ALD reactor control and in situ characterization into a single software program is not uncommon across academic and industrial ALD laboratories, but little has been previously published on the nuts and bolts of such endeavors. A fully functional Simulation Mode was added to the VI to troubleshoot while programming, and it was ultimately left in the program to allow for demonstration of the ALD reactor operation during personnel training or laboratory tours. Figure 2.3 shows a screenshot of the VI 
front panel. The VI allows the user to initialize and manually control all reactor components, including valves, mass flow controllers (MFCs), the in-situ QCM sensor, and the reactor furnace temperature controller (pending incorporation of instrument drivers). Default precursor names, needle valve settings, and thermocouple locations are automatically loaded from setup files. User modifications made to any of these parameters during VI operation are propagated throughout the program and noted in the $\log$ files when an ALD experiment is performed. Precursors configured in the setup files but set to be disabled have their corresponding front-panel controls disabled and greyed out at run-time, as shown in Figure 2.4. Though only 9 chemical precursors are currently installed on the ALD reactor and the National Instruments compact data acquisition (cDAQ) thermocouple (TC) module in use accommodates just 4 TCs, the program was designed with modularity and future expansion in mind, accommodating valve control of up to sixteen precursors and monitoring of up to twenty TCs. Additional cDAQ modules for this expanded capacity were purchased and will be installed at a later date. Sections of the VI block-diagram code illustrating program modularity and basic execution are provided in Appendix A. 


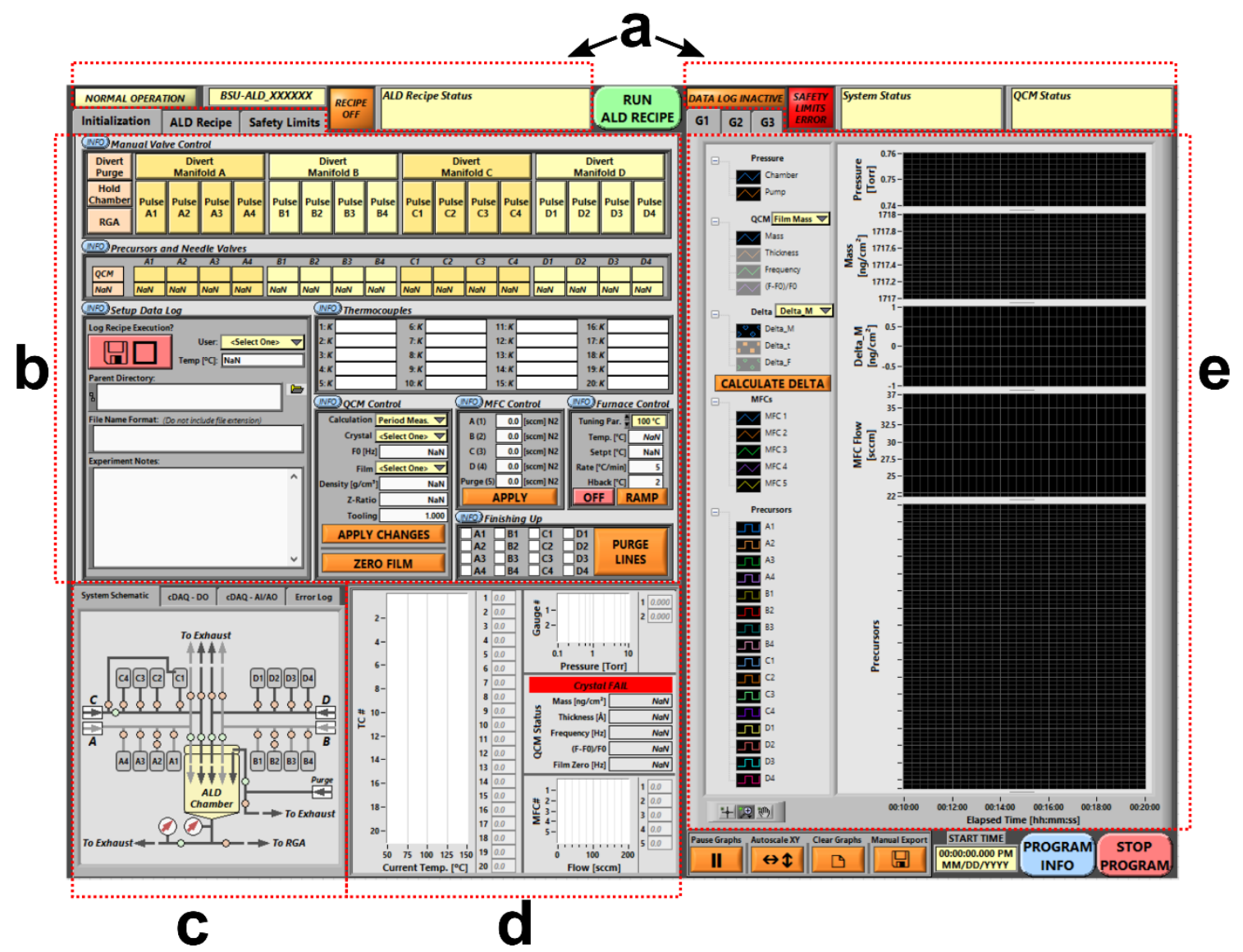

Figure 2.3 Screenshot of LabVIEW software for control and monitoring of ALD reactor operation. Regions highlighted in red boxes are a) ALD recipe and system status indicators, b) ALD reactor controls, c) ALD reactor schematic with valve status indicators, d) real-time display of measured process values, and e) measured and calculated process parameters graphed as a function of time. Enlarged views of highlighted regions b-e are shown during program operation in Figures 2.4, 2.6, and 2.7. 


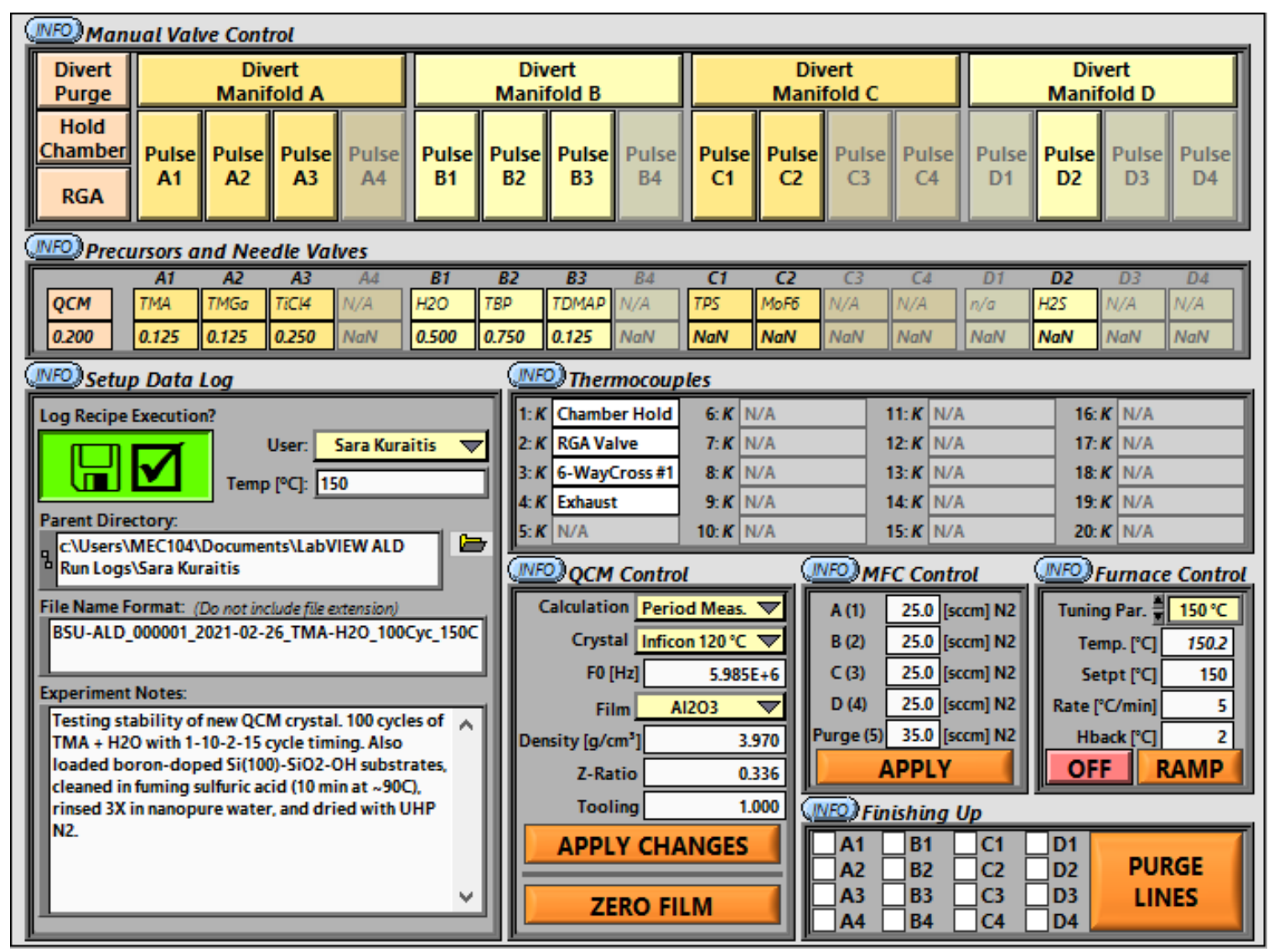

Figure 2.4 In-operation screenshot of Initialization tab for manual control of the ALD reactor, highlighted in region b) of Figure 2.3. Default precursor names and needle valve settings are automatically populated based on initialization cluster arrays containing precursor configuration details (see Appendix A.1).

A key improvement to the VI was implementation of custom user-defined ALD

recipes for automated execution. Instead of limiting the user to a single A-B ALD

chemistry requiring manual input to change process parameters, the VI allows the user to

build a fully customized ALD recipe table with pre-defined parameter changes to be

carried out during recipe execution. Recipe steps can be added or removed from any line

in the recipe, and subsections can also be duplicated and added at a later point in the

table. ALD recipes can also be exported and loaded directly from text files, enabling

complex automated experiments with precise and repeatable conditions. 
Current ALD recipe capabilities include stand-alone purge/wait steps, ALD precursor cycles with up to four precursors (A-B-C-D chemistry) per loop, changes in carrier gas flow, and reactor temperature ramps (pending incorporation of instrument drivers). The program includes three ALD dosing types: full-flow (FF), full-flow with diversion of non-dosing lines (FFD), and fill-hold-purge (FHP) for static dosing. Both the FFD and FHP dosing types serve to increase the relative dose for a given precursor pulse time and are used for ALD chemistries with low reactivity or substrates with large aspect ratios. Each recipe step also allows for activation of a valve leading to a mass spectrometer, which allows for precise correlation of ALD recipe execution with residual gas analysis (RGA) data collection to achieve full-range time-resolved mass spectrometry. ${ }^{23}$ User controls have been added to incorporate recipe steps with Fouriertransform infrared spectroscopy (FTIR) scans. Full implementation of this additional recipe step type in the recipe execution block-diagram code remains to be addressed in future work.

Figure 2.5 shows the ALD recipe controls and an example recipe table. The recipe begins by setting each of the MFCs to 25-35 sccm of nitrogen flow. The reactor temperature is then increased to $130{ }^{\circ} \mathrm{C}$ using a ramp rate of $5{ }^{\circ} \mathrm{C} / \mathrm{min}$, a hold-back of \pm 2 ${ }^{\circ} \mathrm{C}$, and tuning parameters calculated when the temperature controller was tuned to 150 ${ }^{\circ} \mathrm{C}$. Selecting tuning parameters that correspond to a setpoint close to the target temperature enables more accurate and stable temperature control. After the temperature ramp is complete, the system is purged with nitrogen for 20 minutes to reach thermal equilibrium, and then ALD cycles begin execution. In this case, alternating doses of 
trimethylaluminum (TMA) and water $\left(\mathrm{H}_{2} \mathrm{O}\right)$ are performed, and the RGA valve is toggled open during the TMA half-cycle for time-resolved mass spectrometry. ${ }^{23}$

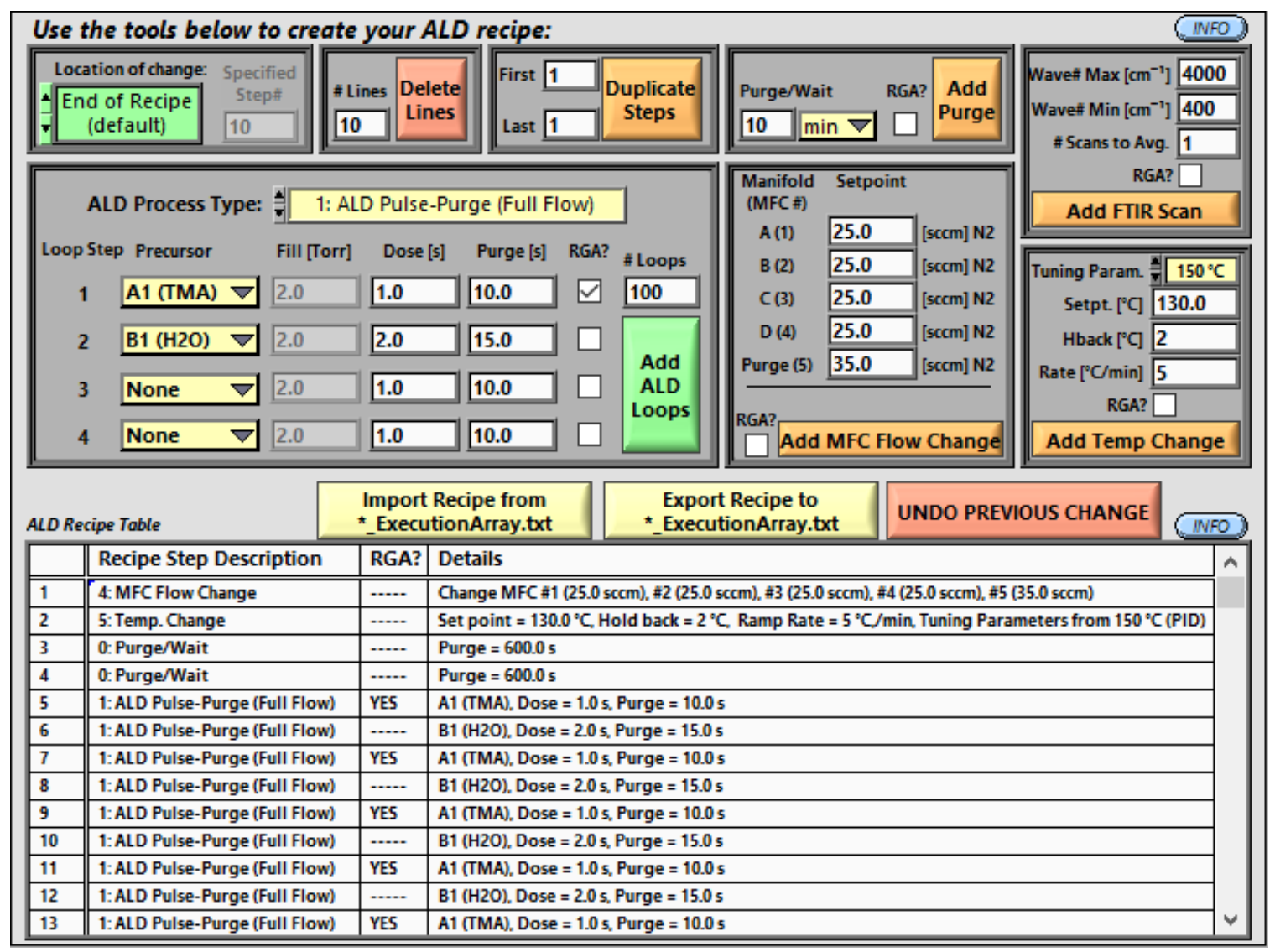

Figure 2.5 Screenshot of user controls for creating customized ALD Recipe Tables in the LabVIEW program. In addition to standard ALD precursor cycles, recipes may include automated changes to temperature and carrier gas flow. All recipe step types also include the option to toggle a pneumatic valve for sampling of residual reaction gases using a mass spectrometer. Future modifications will include implementation of automated FTIR scans, which have already been included in the front-panel ALD Recipe controls.

Figure 2.6 shows the in-operation indicators for valve status and instantaneous parameter values, graphed as bar plots to aid in visual recognition of any out-of-spec elements. The configuration illustrated in Figure 2.6 corresponds to the "Hold" step of an FHP ALD cycle, with all gas delivery lines diverted to exhaust while the reaction chamber is held static. Because QCM use requires constant gas flow to the sensor head to 
prevent film deposition on the back of the crystal, QCM is not compatible with FHP processes, as discussed in sections 2.3 and 2.4; thus, the QCM status shown in Figure 2.6 indicates no QCM is in use.

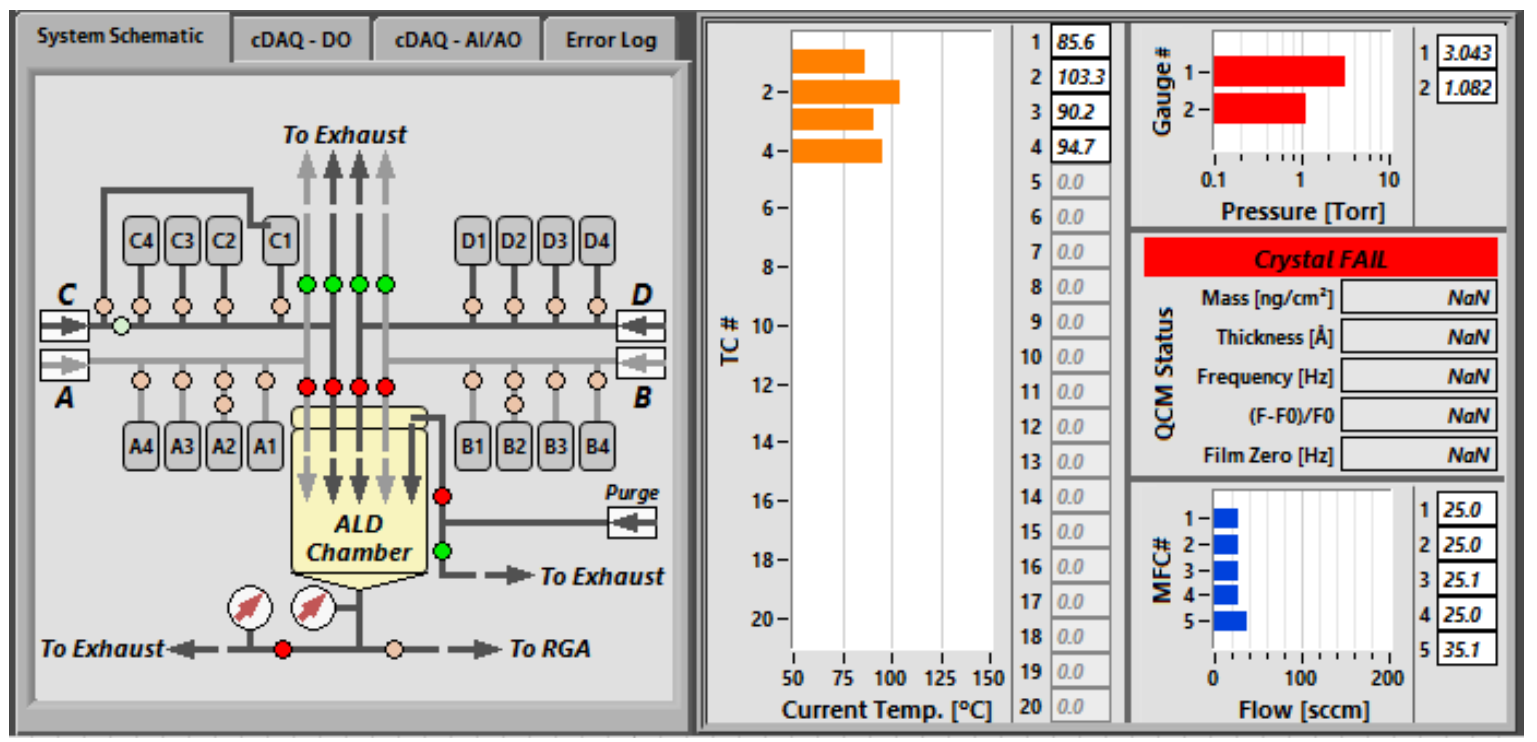

Figure 2.6 In-operation screenshot of valve status indicators and instantaneous process parameter values, highlighted in regions c-d) of Figure 2.3. The system is shown with all gas delivery lines diverted to exhaust and the chamber being held static, corresponding to the Hold recipe step in a Fill-Hold-Purge ALD cycle.

Another key addition to this VI was the calculation of process-dependent film properties using real-time data from in situ process metrology tools, including real-time computation and display of stepwise film mass changes $(\Delta \mathrm{m})$ resulting from each ALD half cycle. Data post-processing is both time consuming and subject to significant errors, since it can be difficult to re-synchronize the process control states with the metrology data record; furthermore, reactions with little or no net mass change cannot be clearly observed in the QCM data and can be virtually impossible to identify if the QCM record is not synchronized with precursor valve operation. Computing these mass changes from the recorded QCM data in real-time as each ALD half cycle is completed eliminates such post-processing challenges and allows for immediate process diagnostics and evaluation. 
The program automatically performs $\Delta \mathrm{m}$ calculations during ALD recipe execution, and also includes functionality for the user to trigger $\Delta \mathrm{m}$ calculations when manually operating the reactor. Provided the user has entered appropriate values for film density, the program also calculates stepwise film thickness changes $(\Delta \mathrm{t})$ corresponding to each $\Delta \mathrm{m}$ value. Figure 2.7 shows in-operation graphs for both measured and calculated process parameters. The data shown were produced in Simulation Mode during program development. Pressure, QCM film mass, calculated $\Delta \mathrm{m}$ values, carrier gas flow, and precursor dosing status are all displayed on the G1 graphs tab. Additional tabs (not shown) provide similar graphs for 20 TCs and all digital-output cDAQ lines.

To mitigate risks associated with accidental release of chemical precursors in the event of an equipment malfunction, an action engine VI was implemented to continuously monitor reactor parameters (i.e., pressure, MFC flow, and temperature) and to limit VI control and recipe execution capabilities if those measured values fall outside acceptable ranges. Figure 2.8 shows a screenshot of the front-panel controls used to set safety limits. In manual operation mode, these safety limits prevent the user from dosing precursors into the reactor or from starting an ALD recipe. Should measured parameters fall outside the safety limits while an ALD recipe is in progress, the recipe is automatically terminated, and the precise time and reason for termination are noted in the appropriate log file. The user must then reset the safety limit errors to re-enable ALD recipe execution or manual precursor dosing. 


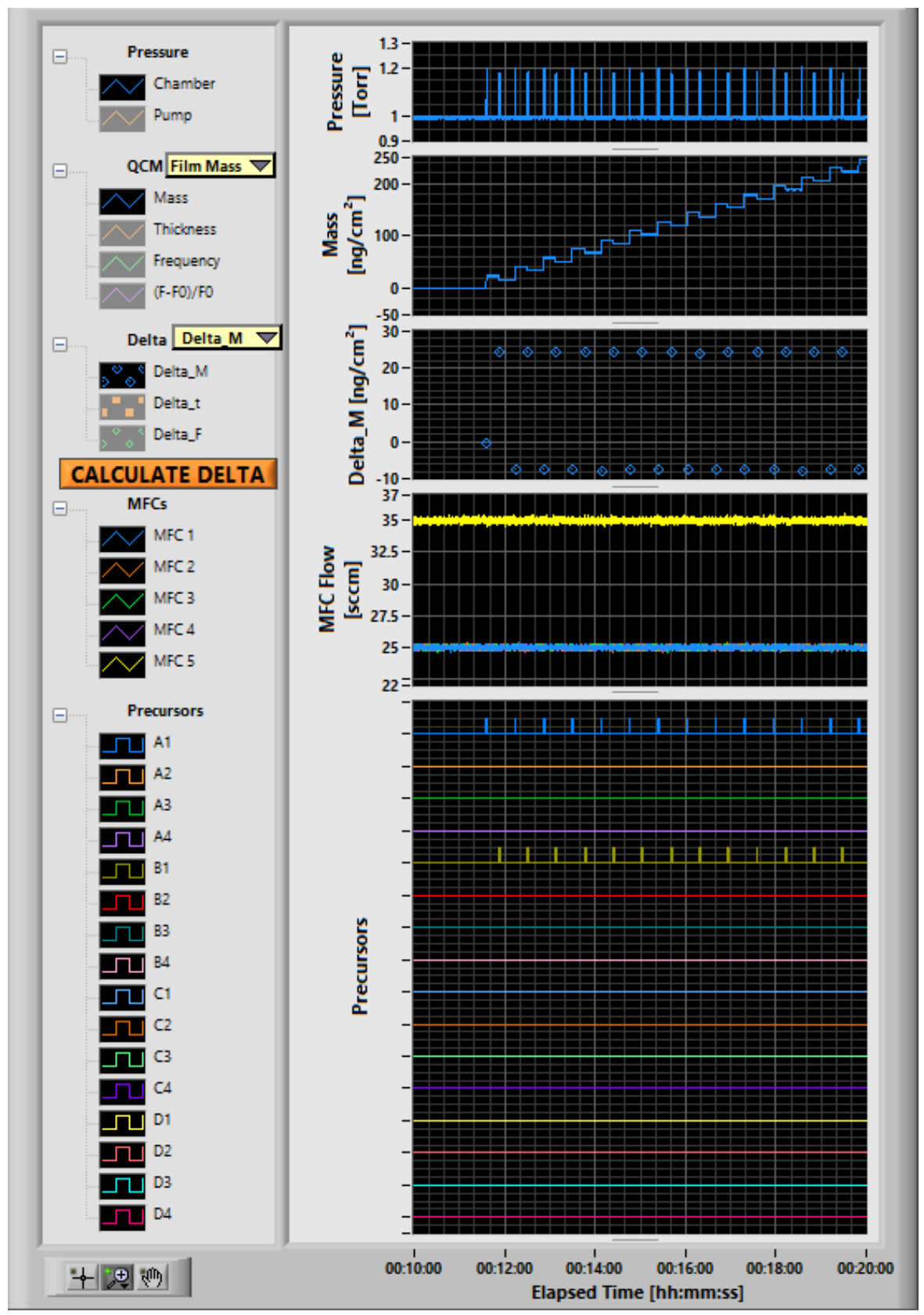

Figure 2.7 In-operation screenshot of LabVIEW front-panel graphs on the G1 graphs tab, highlighted in region e) of Figure 2.3. The data shown were produced in Simulation Mode during program development. G2 and G3 tabs (not shown) contain graphs for 20 TCs and all digital-output cDAQ lines, respectively. 


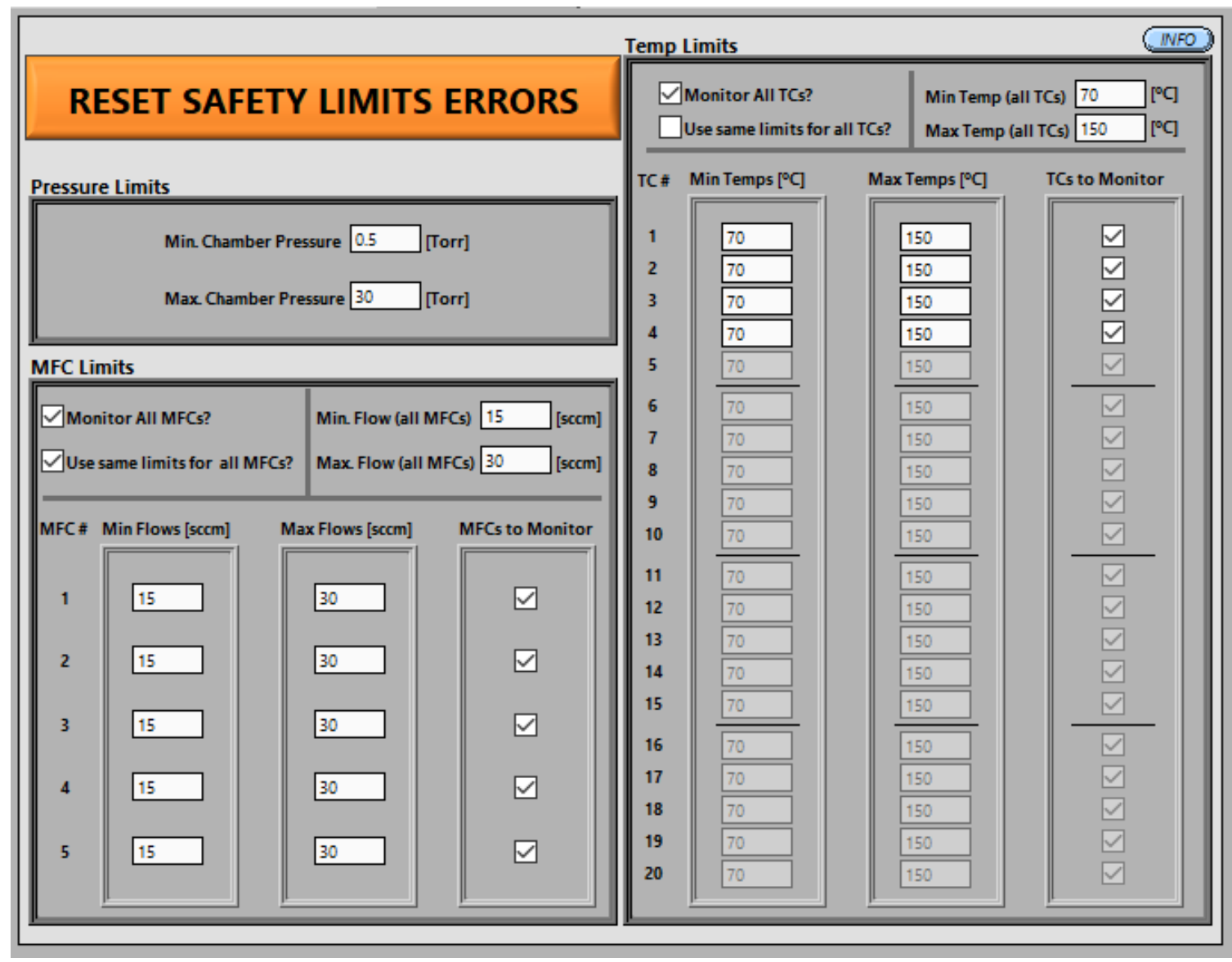

Figure 2.8 Screenshot of user controls for setting safety limits, which prevent reactor operation if pressure, carrier gas flow, or temperature measurements fall outside acceptable ranges. Safety limits can be set to experiment-specific values as needed (i.e., higher temperature requirements for low vapor pressure precursors). Elements disabled in the setup file cluster arrays are automatically disabled and greyed out at run-time, as discussed in Appendix A.

In the investigation of GaP ALD described in the remainder of this chapter, substrate deposition runs were performed prior to the re-design of the ALD reactor and improved LabVIEW program. QCM runs were performed after modifications to the ALD reactor were completed, but after only preliminary improvements were made to the LabVIEW program. Full implementation of the LabVIEW program improvementsincluding incorporation of MFC flow changes, temperature ramps, RGA valve control, and real-time $\Delta \mathrm{m}$ calculations-were completed in anticipation of the future work outlined in the conclusion of this chapter. 


\subsection{Characterization of GaP ALD: Experimental Methods}

\section{$\underline{\text { 2.3.1 ALD of GaP }}$}

TMGa (>99\%, Strem Chemicals) and TBP ( $\sim 95 \%$, Strem Chemicals) were contained in stainless-steel cylinders (Swagelok), while TDMAP ( $>96 \%$, TCI America or Sigma Aldrich) was contained in a glass vessel and heated to $\sim 60{ }^{\circ} \mathrm{C}$ using a miniature benchtop temperature controller (Omega) to increase vapor pressure. ${ }^{2,3}$ Precursors were delivered to the reactor using $\mathrm{N}_{2}$ or Ar carrier gas, with each of the reactor's five MFCs set to 20-30 sccm to reach a base pressure of $\sim 1$ Torr.

Si(100) substrates with native oxide were cleaned using either Acetone-EthanolWater sonication or a fuming sulfuric acid bath $\left(\mathrm{H}_{2} \mathrm{SO}_{4}\right.$, heated to $\sim 90{ }^{\circ} \mathrm{C}$ for $\left.15 \mathrm{~min}\right)$ followed by nanopure water rinse. A subset of experiments was performed on $\operatorname{Si}(100)$ with native oxide pre-coated with either ALD alumina using alternating doses of trimethylaluminum and water ("TMA $+\mathrm{H}_{2} \mathrm{O}$ ") or ALD titania using alternating doses of titanium tetrachloride and water (“ $\mathrm{TiCl}_{4}+\mathrm{H}_{2} \mathrm{O}$ "). To investigate epitaxial growth on hydrogen-terminated $\mathrm{Si}(100)(\mathrm{H}-\mathrm{Si})$, native oxide was removed from $\mathrm{H}_{2} \mathrm{SO}_{4}$-cleaned substrates via a 10-minute dip in dilute hydrofluoric acid (3\% HF, 97\% water, Ricca Chemical), followed by a brief rinse in nanopure water before being loaded directly into the ALD reactor.

ALD experiments were performed at deposition temperatures of $400-500{ }^{\circ} \mathrm{C}$ in a custom-built ALD reactor with a $\sim 42 \mathrm{~mm}$ ID quartz process tube. Substrates were loaded into the chamber using a cleaved Si platform inside a $\sim 20 \mathrm{~mm}$ ID quartz transfer tube. All precursor delivery lines and exhaust lines were heated to $80-100{ }^{\circ} \mathrm{C}$ to mitigate precursor condensation. 


\subsubsection{Characterization}

Substrates were coated with 100 cycles of ALD GaP film using the Fill-HoldPurge ALD process mode, holding doses static to increase precursor exposure time. Nominal target doses were typically $\sim 10^{6} \mathrm{~L}\left(1 \mathrm{~L}=10^{-6} \mathrm{Torr} \cdot \mathrm{s}\right)$ for the TMGa + TDMAP chemistry, and $\sim 10^{7} \mathrm{~L}$ for the TMGa + TBP chemistry; however, overshoot of the target fill pressure varied widely between experiments before modifications to the ALD reactor and LabVIEW control program were completed, and actual precursor doses were estimated to be up to an order of magnitude higher. Following film deposition experiments, substrates were transferred to and stored in a desiccator to reduce oxidation prior to ex situ characterization. Scanning electron microscopy (SEM) images and energy-dispersive x-ray spectroscopy (EDS) data were collected on an FEI Teneo FESEM equipped with an in-column secondary electron detector, a backscattered electron detector, and Everhart-Thornley detector, and an Oxford Instruments X-Max EDS system ( $80 \mathrm{~mm}^{2}$ detector). EDS data were collected and analyzed using Oxford Instruments AZtec software. Atomic force microscopy (AFM) images of GaP film surfaces were obtained on a Bruker Dimension FastScan AFM equipped with Nanoscope V Controllers operating in peak-force tapping mode using ScanAsyst-Air-HR probes in ambient conditions. X-ray diffraction (XRD) data were collected on a Rigaku MiniFlex 600 Benchtop x-ray diffractometer. X-ray photoelectron spectroscopy (XPS) data were collected on a PHI 5600 with a base pressure of $2 \times 10^{-10}$ Torr using a focused Al Ka xray source. Spectroscopic ellipsometry (SE) was used to investigate film thickness and optical properties. SE measurements were performed on a J.A. Woollam M-2000 ellipsometer and analyzed using CompleteEASE 5.10 software. 
In situ QCM measurements were performed in full-flow ALD dosing mode using a welded ALD sensor (Inficon) with a gallium phosphate crystal that was temperaturecompensated at $500{ }^{\circ} \mathrm{C}\left(\mathrm{R}-30 \mathrm{Y}-11.1^{\circ} \mathrm{GaPO}_{4}\right.$ resonator, Piezocryst) and an STM-2 Thin Film Rate/Thickness Monitor (Inficon). The ALD sensor head was continually purged with $\mathrm{N}_{2}$ gas to prevent film deposition on the back side of the $\mathrm{GaPO}_{4}$ crystal. Because of this need for continuous $\mathrm{N}_{2}$ purge, QCM experiments could not be performed under the Fill-Hold-Purge ALD dosing mode to replicate conditions used during substrate deposition experiments. A small number of quasi-FHP QCM runs (precursor lines diverted but QCM back-purge still flowing) were also completed for the TMGa + TBP chemistry. GaP growth on various oxides was investigated by pre-coating the $\mathrm{GaPO}_{4}$ crystal with ALD silica using tris(tert-pentoxy)silanol (TPS), ${ }^{24}$ ALD alumina (TMA + $\mathrm{H}_{2} \mathrm{O}$ ), or ALD titania $\left(\mathrm{TiCl}_{4}+\mathrm{H}_{2} \mathrm{O}\right)$. Mass changes per unit area were calculated from measured resonator frequency according to the Sauerbrey equation, as output by the STM-2 LabVIEW library supplied by Inficon.

\subsection{Characterization of GaP ALD: Results and Discussion}

Typical SEM results for ALD GaP film deposited using 150 cycles of TMGa + TDMAP with nominal $\sim 10^{6} \mathrm{~L}$ target precursor doses on $\mathrm{SiO}_{2} / \mathrm{Si}(100)$ at $450^{\circ} \mathrm{C}$ are shown in Figures 2.9 and 2.11, and AFM results are shown in Figure 2.10. GaP film was uniform and smooth (RMS roughness of $\sim 0.85 \mathrm{~nm}$ ), with 3D rod-like structures

appearing on top of the film as shown in Figure 2.11. EDS results (Figure 2.12) indicated both film and 3D rod-like structures deposited via TMGa + TDMAP were composed of $\mathrm{GaP}$. 
Spectroscopic ellipsometry (SE) data (not shown) was collected to investigate film optical properties, but the data could not be fit to optical models for GaP, gallium oxide, or other likely film compositions. The poor-quality SE data may have been due to a combination of effects from the $3 \mathrm{D}$ rod-like structures scattered across the sample surface and from contamination with precursor decomposition byproducts. Consequently, SE could not be used to calculate GaP film thickness to validate the QCM growth-percycle (GPC) calculations described later.

Similar results were observed for ALD GaP film deposited using 150 cycles of $\mathrm{TMGa}+\mathrm{TBP}$ with $\sim 10^{7} \mathrm{~L}$ precursor doses on $\mathrm{SiO}_{2} / \mathrm{Si}(100)$ at $450{ }^{\circ} \mathrm{C}$, as shown in Figure 2.13. EDS area spectra (Figure 2.14), XPS (Figure 2.15), and XRD (Figure 2.16) confirmed films were composed of polycrystalline GaP.

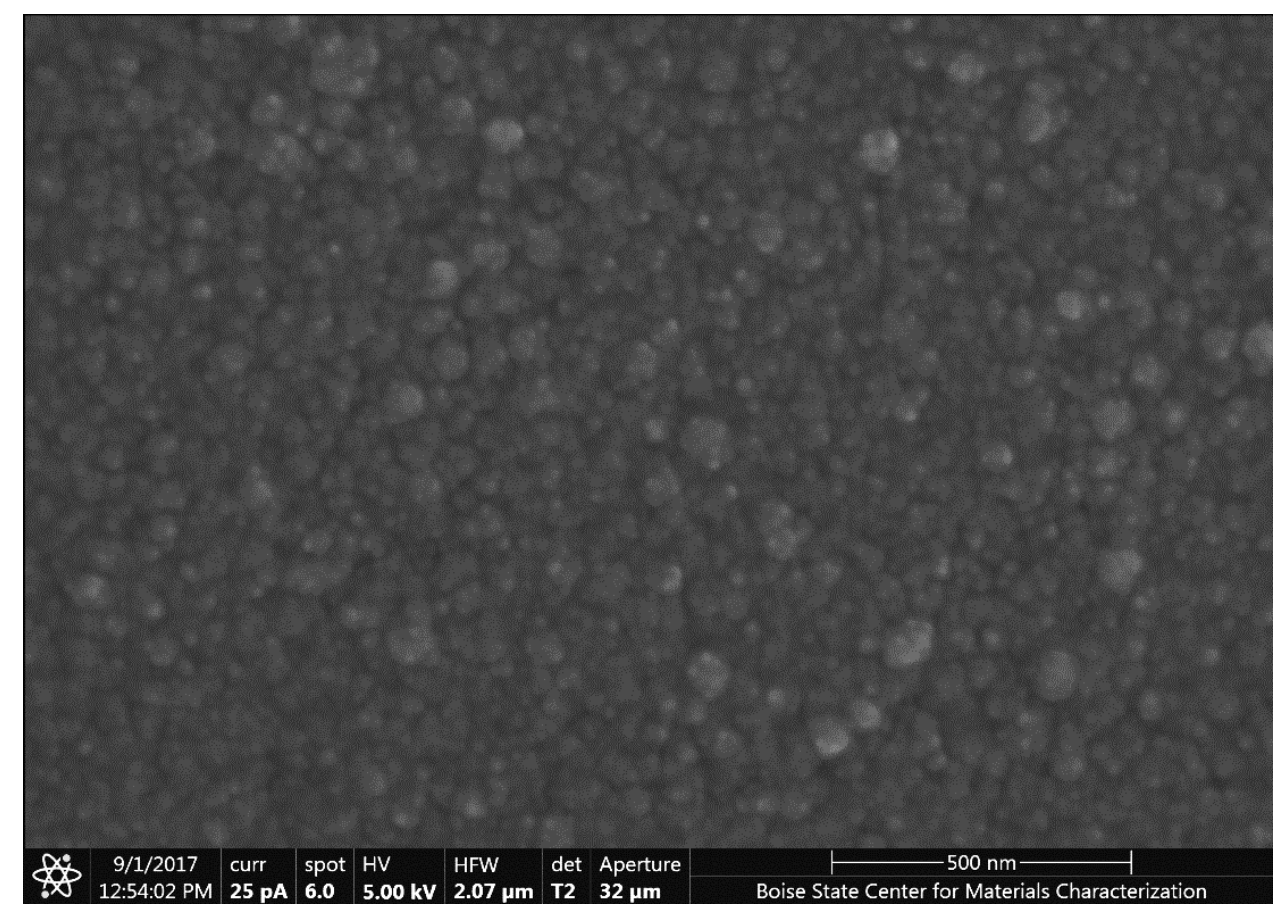

Figure 2.9 High resolution $\mathrm{SEM}$ image of $\mathrm{GaP}$ film grown on $\mathrm{SiO}_{2} / \mathrm{Si}(100)$ at 450 ${ }^{\circ} \mathrm{C}$ using 150 cycles of TMGa + TDMAP. 


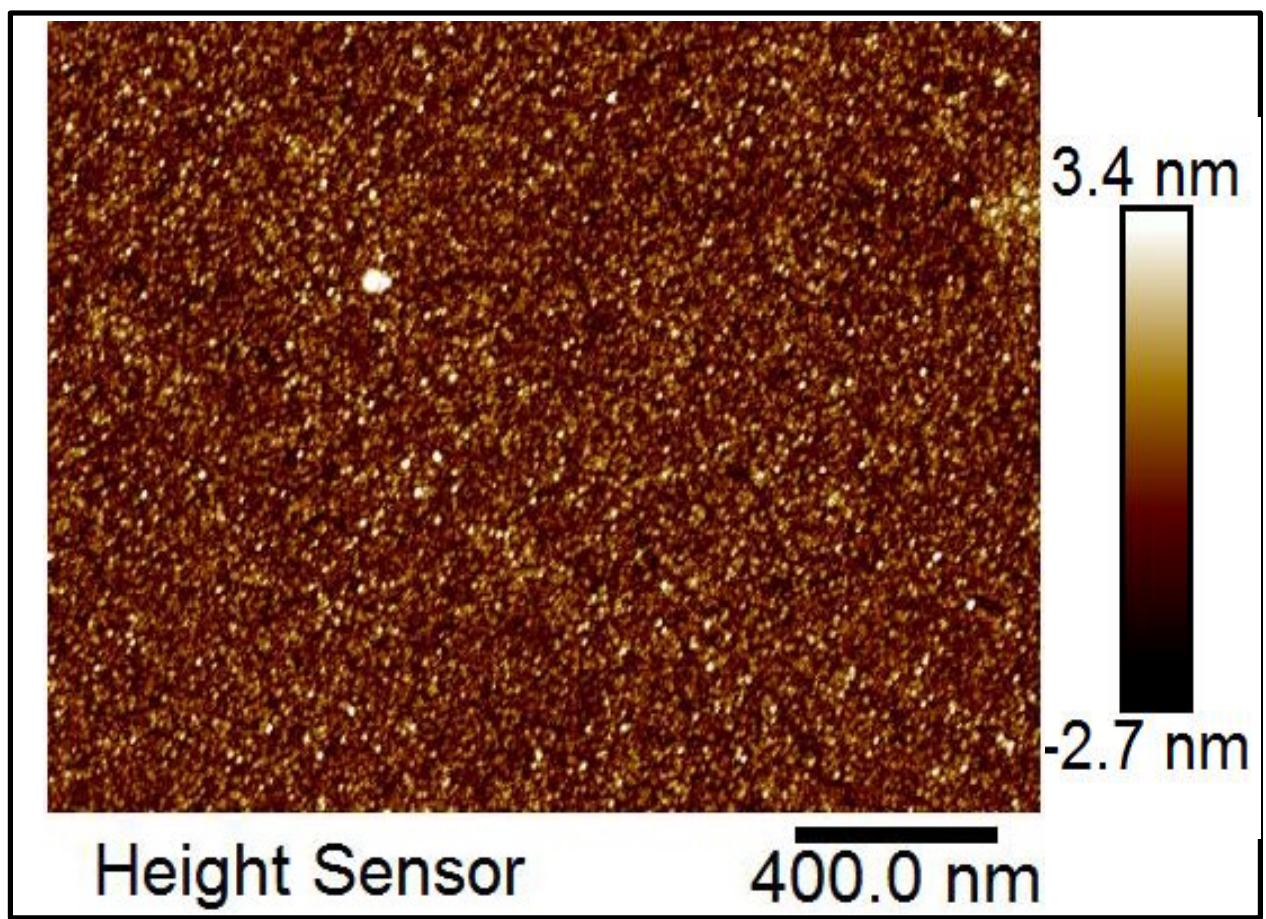

Figure 2.10 AFM topography of GaP film grown on $\mathrm{SiO}_{2} / \mathrm{Si}(100)$ at $450{ }^{\circ} \mathrm{C}$ using 150 cycles of TMGa + TDMAP. RMS surface roughness was $\sim 0.85 \mathrm{~nm}$.

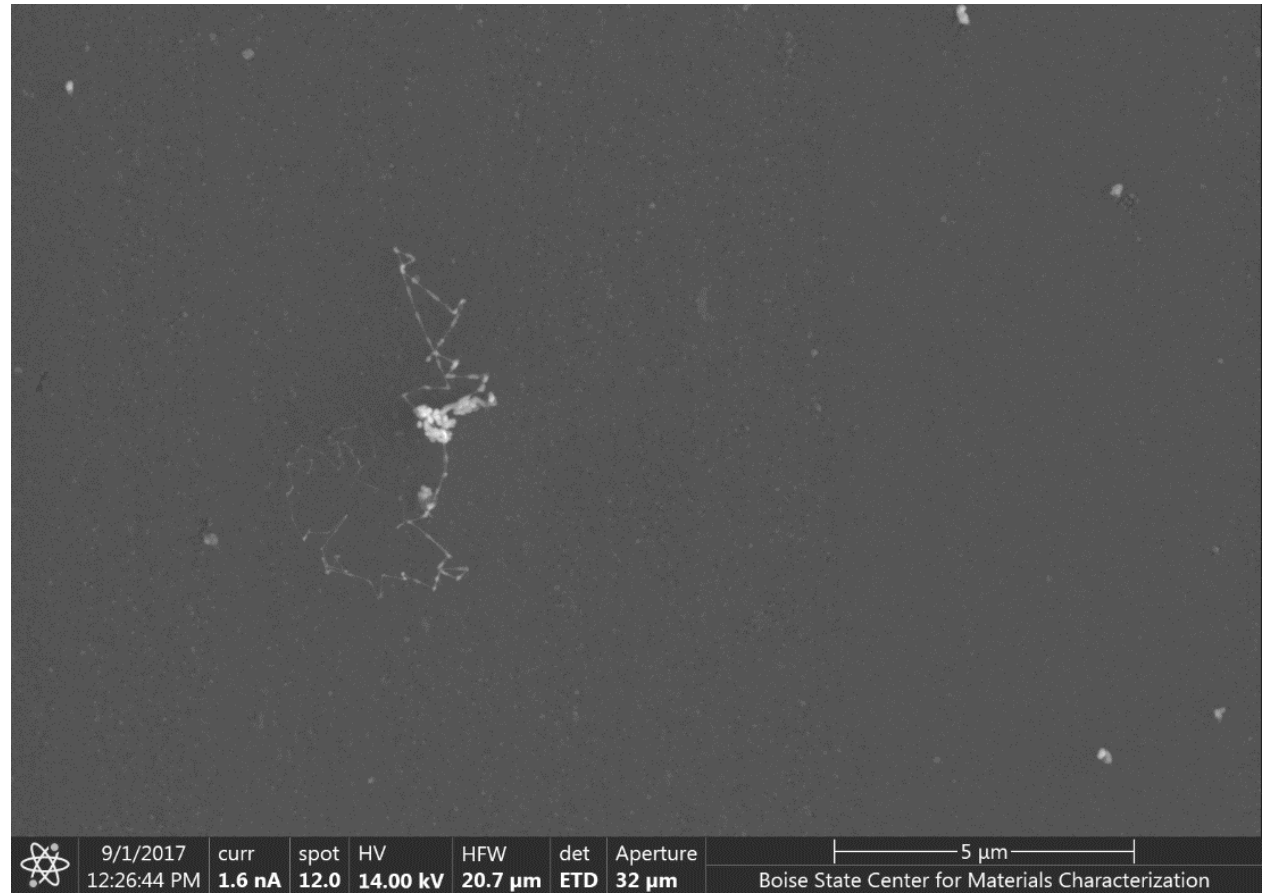

Figure 2.11 $\mathrm{SEM}$ image of GaP film grown on $\mathrm{SiO}_{2} / \mathrm{Si}(100)$ at $450{ }^{\circ} \mathrm{C}$ using 150 cycles of TMGa + TDMAP. EDS data for this sample are provided in Figure 2.12. 

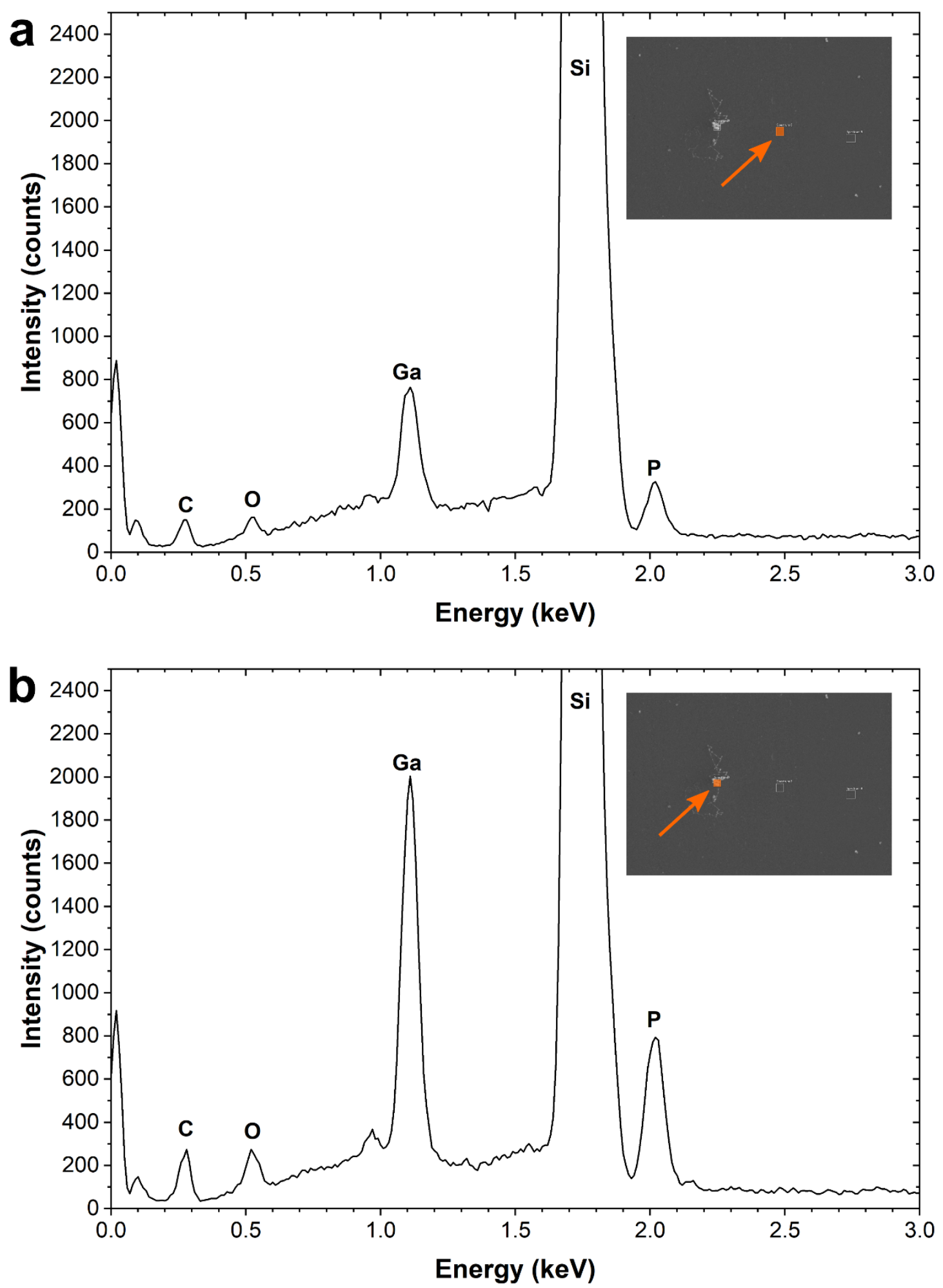

Figure 2.12 EDS spectra of ALD GaP a) film and b) 3D rod-like structure grown on $\mathrm{SiO}_{2} / \mathrm{Si}(100)$ at $450{ }^{\circ} \mathrm{C}$ using 150 cycles of TMGa + TDMAP. Inset electron images show corresponding regions of the sample pictured in Figure 2.11 scanned for EDS area spectra. 


\section{Electron Image 7}

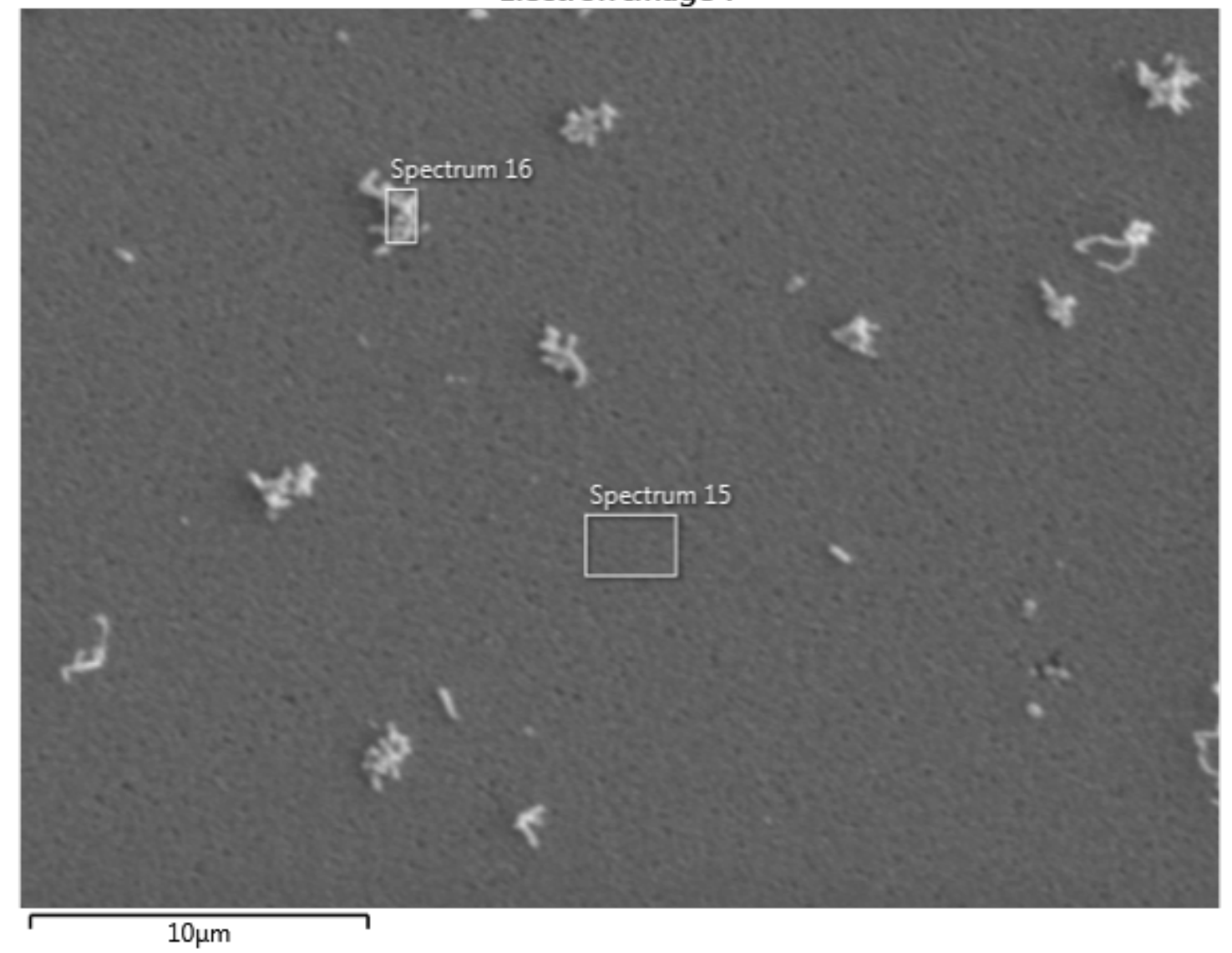

Figure 2.13 $\mathrm{SEM}$ image of GaP film grown on $\mathrm{SiO}_{2} / \mathrm{Si}(100)$ at $450{ }^{\circ} \mathrm{C}$ using 150 cycles of TMGa + TBP. EDS data for this sample are provided in Figure 2.14. 

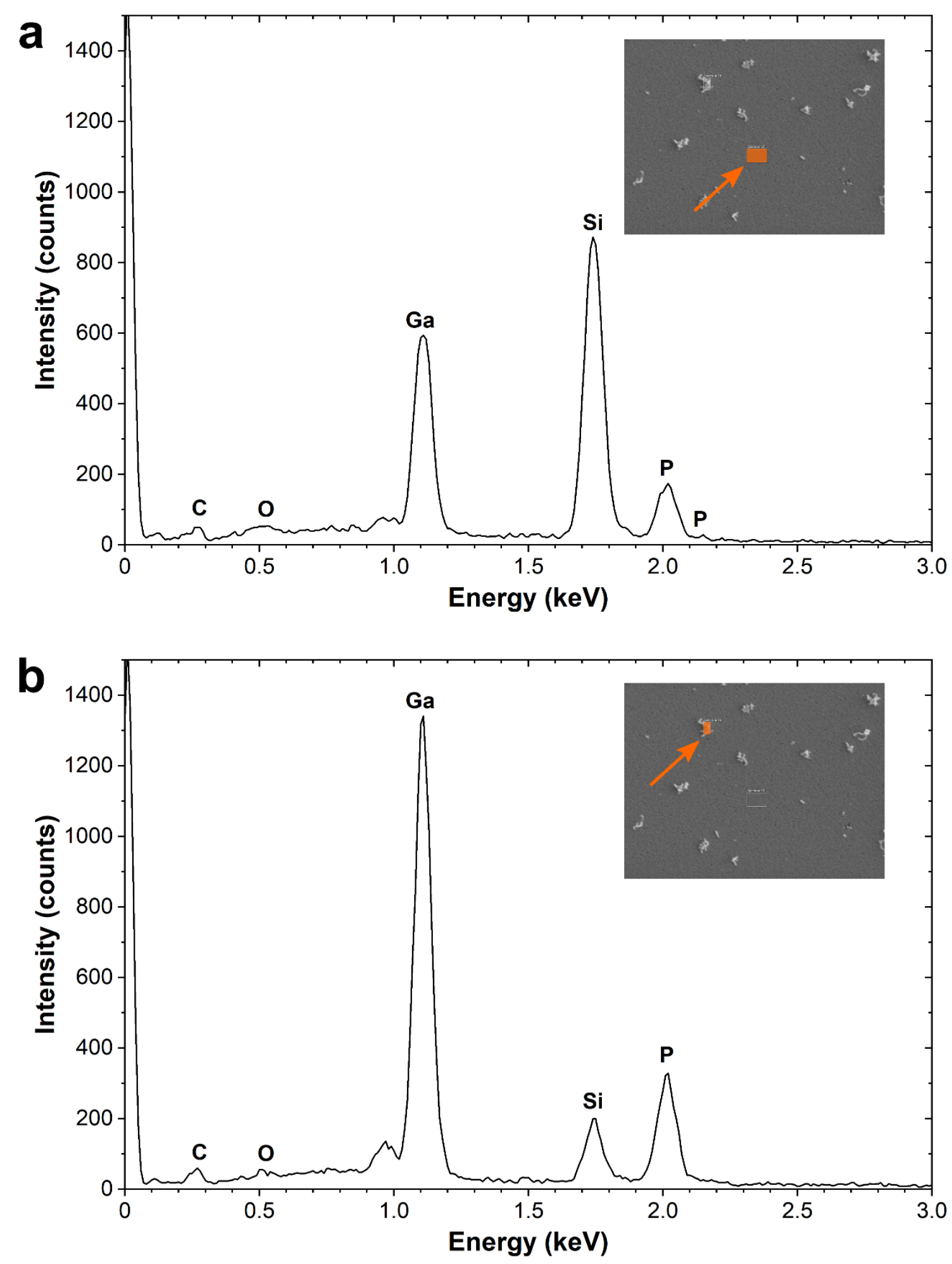

Figure 2.14 EDS spectra of ALD GaP a) film and b) 3D rod-like structure grown on $\mathrm{SiO}_{2} / \mathrm{Si}(100)$ at $450{ }^{\circ} \mathrm{C}$ using 150 cycles of $\mathrm{TMGa}+\mathrm{TBP}$. Inset electron images show corresponding regions of the sample pictured in Figure 2.13 scanned for EDS area spectra. 


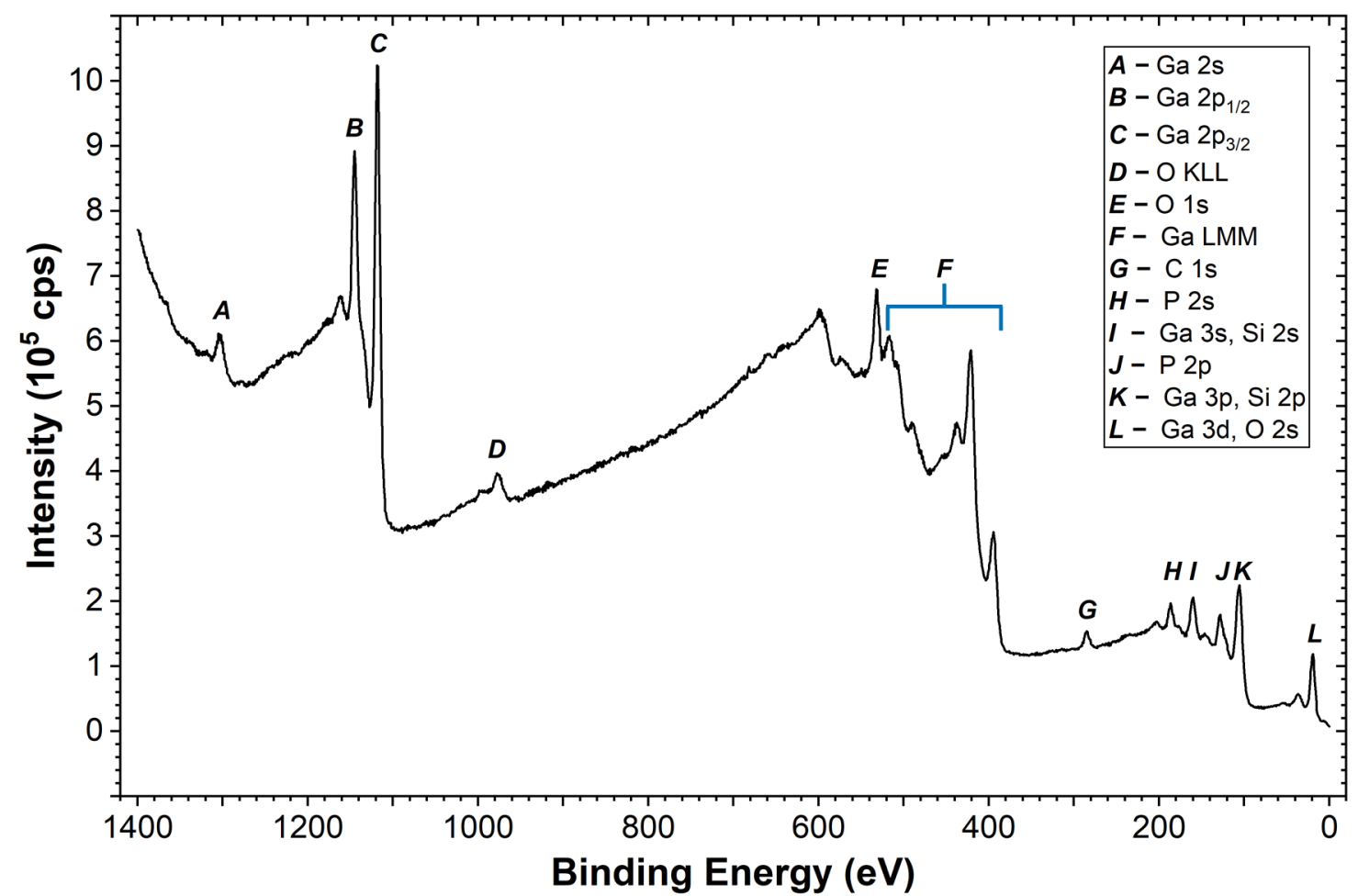

Figure 2.15 XPS of GaP film grown on $\mathrm{SiO}_{2} / \mathrm{Si}(100)$ at $450{ }^{\circ} \mathrm{C}$ using 150 cycles of TMGa + TBP. 


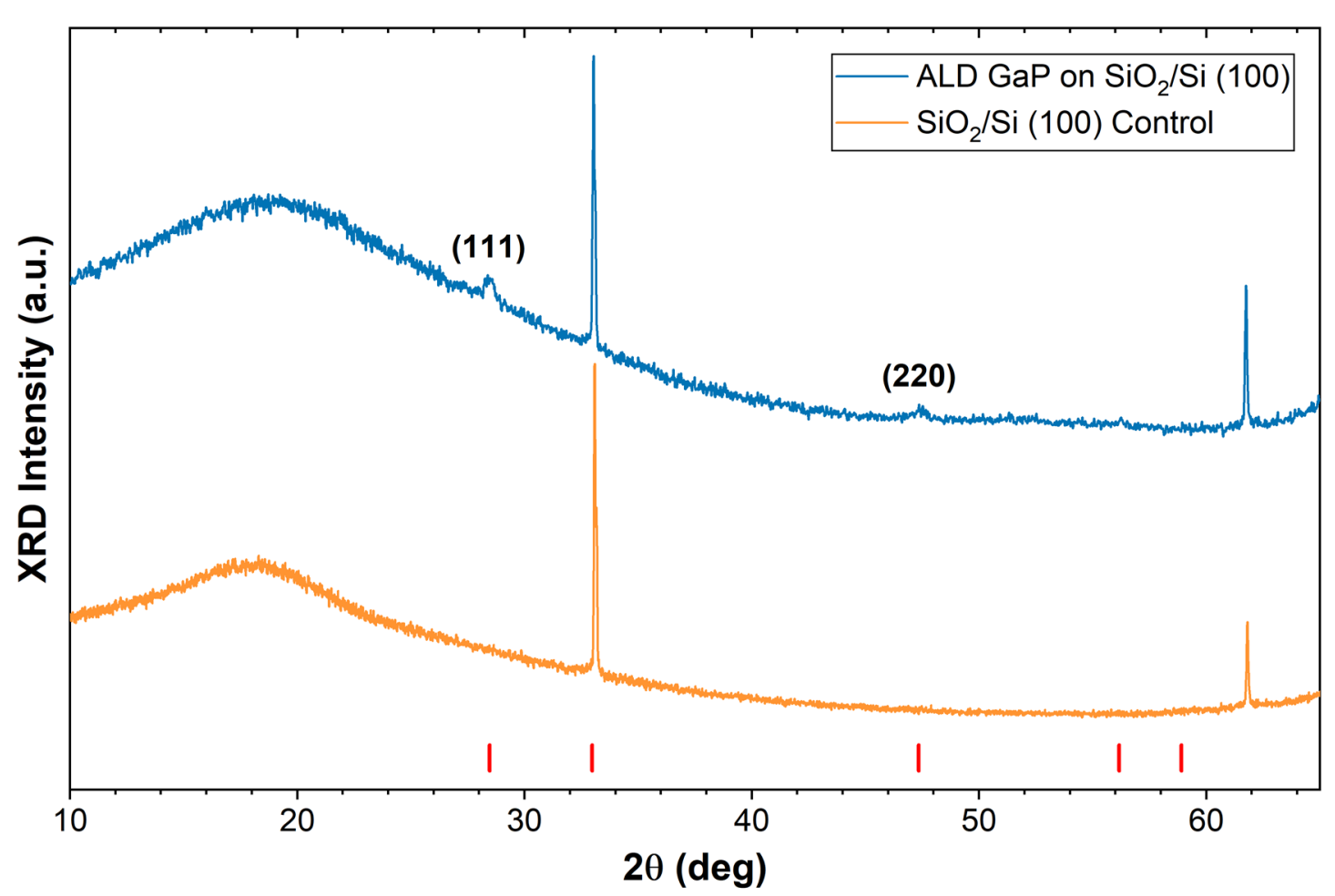

Figure 2.16 XRD scan of GaP film grown on $\mathrm{SiO}_{2} / \mathrm{Si}(100)$ at $450{ }^{\circ} \mathrm{C}$ using 150 cycles of TMGa + TBP. Presence of GaP (111) and GaP (220) peaks indicates films were polycrystalline GaP. Red hash marks shown for GaP reflections from ICDD PDF 00-032-0397.

Although Figures 2.9-2.16 illustrate successful GaP deposition with only a small number of non-ideal 3D structures scattered across the surface, consistent results were difficult to achieve. In other TMGa + TDMAP experiments using the same substrate preparation steps and ALD process parameters, films showed large amounts of variation ranging from minimal film growth to complex rod- or pollen-like structures covering the substrate surfaces. The shape, and size, and density of 3D GaP structures was found to correlate with both temperature and substrate cleanliness. Figures 2.17-2.19 show SEM results for ALD GaP film deposited using 150 cycles of TMGa + TDMAP with nominal $\sim 10^{6} \mathrm{~L}$ target precursor doses on $\mathrm{SiO}_{2} / \mathrm{Si}(100)$ at $475^{\circ} \mathrm{C}$. Although the substrate surface was still coated with GaP film, both the size and spatial density of 3D GaP structures increased with elevated growth temperature. 
Substrate cleanliness was determined to be of paramount importance for both the $\mathrm{TMGa}+\mathrm{TDMAP}$ and TMGa + TBP chemistries. Figures 2.20-2.23 show several variations of the $3 \mathrm{D} \mathrm{GaP}$ growth modes observed on $\mathrm{SiO}_{2} / \mathrm{Si}(100)$ at $450{ }^{\circ} \mathrm{C}$ with nominal target precursor TMGa + TBP doses of $\sim 10^{7}$ L. Substrates were cleaned in fuming sulfuric acid and then stored for several weeks prior to being used in a deposition experiment. Some regions showed small rod-like structures spread across uniform GaP film (Fig. 2.20). Other sample regions showed smooth film with large spherical agglomerations of rod-like structures (Fig. 2.21). One large region on the same sample contained large spherical agglomerations of rod-like structures upon a "carpet" of smaller rods (Fig. 2.22). The exact nature of the contamination sensitivity was not identified, and reproducibility remained a persistent issue despite herculean efforts to fine-tune the substrate preparation process and ALD reactor conditions; however, $\mathrm{H}_{2} \mathrm{SO}_{4}$-cleaned samples that were placed in the reactor chamber immediately after being rinsed with nanopure water and dried with UHP $\mathrm{N}_{2}$ gas showed a tendency toward improved film quality and a lesser degree of 3D growth.

A small number of preliminary experiments were performed with TMGa + TBP deposition at $450{ }^{\circ} \mathrm{C}$ on $\mathrm{H}-\mathrm{Si}(100)$ to investigate epitaxial $\mathrm{GaP}$ growth analogous to that using triethylgallium (TEGa) + TBP briefly described in the literature. ${ }^{4,5}$ The preliminary $\mathrm{TMGa}+\mathrm{TBP}$ experiments yielded films that appeared smoother and more uniform to the eye, with visible contrast relative to films grown on $\mathrm{SiO}_{2}-\mathrm{Si}(100)$ substrates in the same ALD run. Unfortunately, the H-Si(100) substrates could not be fully characterized due to public health concerns that necessitated laboratory closures and are therefore not shown here. Subsequent experiments yielded inconsistent results. Further investigation of TMGa 
+ TBP growth on H-Si may be of interest for both general applications of GaP thin films, as well as possible area-selective behavior.

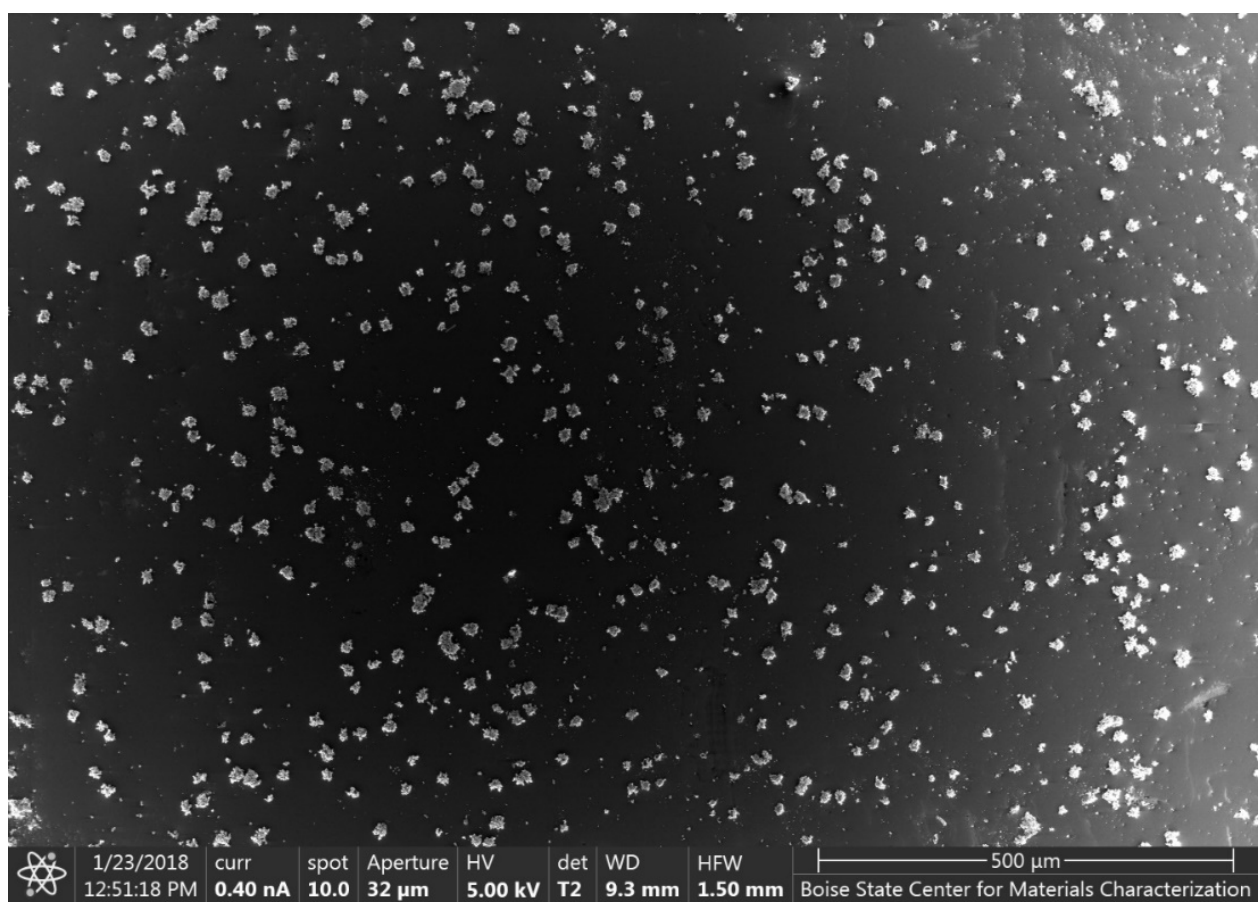

Figure 2.17 $\mathrm{SEM}$ image of $\mathrm{GaP}$ film grown on $\mathrm{SiO}_{2} / \mathrm{Si}(100)$ at $475^{\circ} \mathrm{C}$ using 150 cycles of TMGa + TDMAP, showing an increased number of rod-like 3D structures on top of the surface film. A high-resolution image (50X increase in magnification) of the center of this region is provided in Figure 2.18, and a cross-sectional image of the sample is provided in Figure 2.19. 


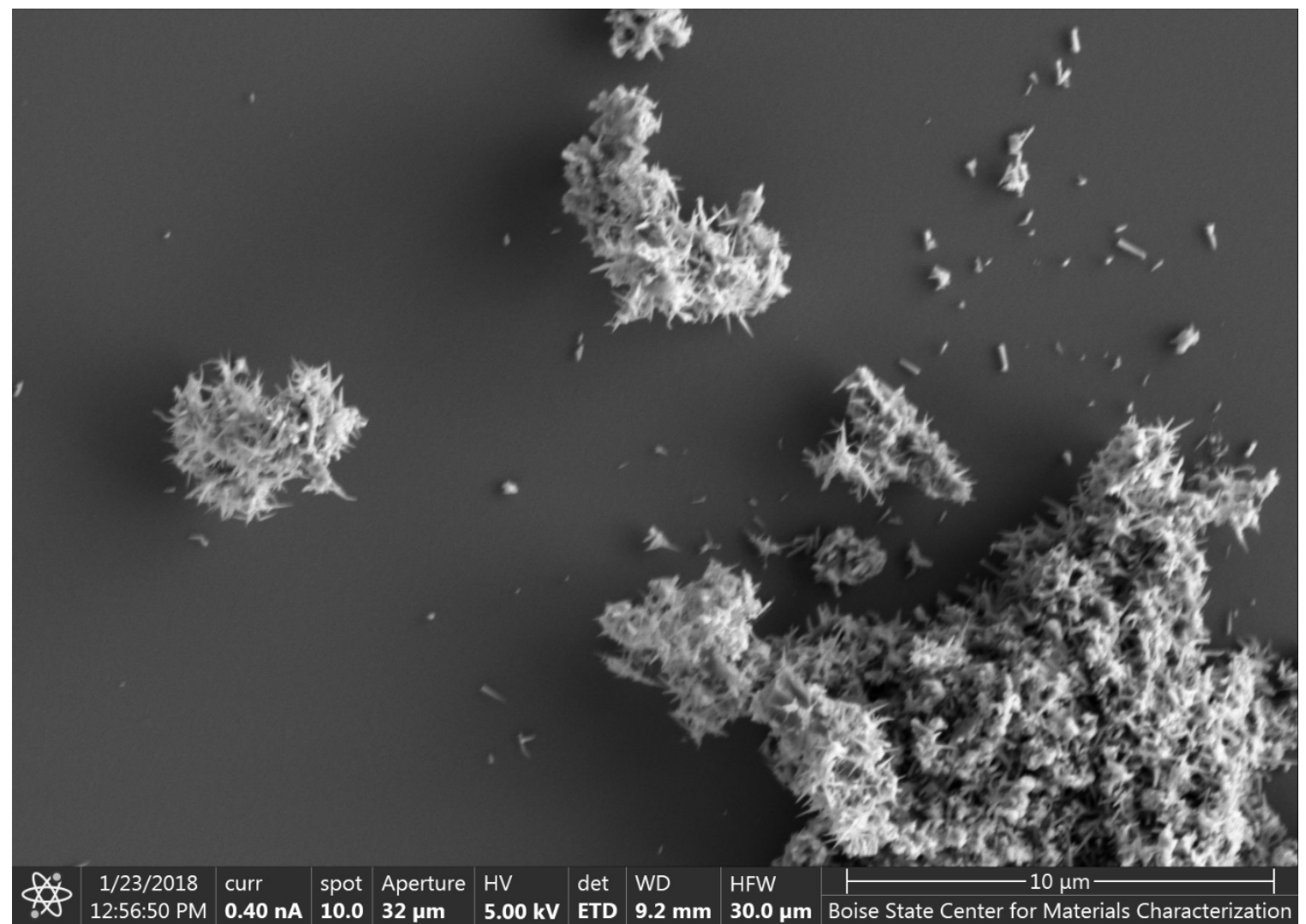

Figure 2.18 SEM image of $\mathrm{GaP}$ film grown on $\mathrm{SiO}_{2} / \mathrm{Si}(100)$ at $475^{\circ} \mathrm{C}$ using 150 cycles of TMGa + TDMAP, showing detail view of 3D GaP structures (50X increase in magnification relative to Figure 2.17). 


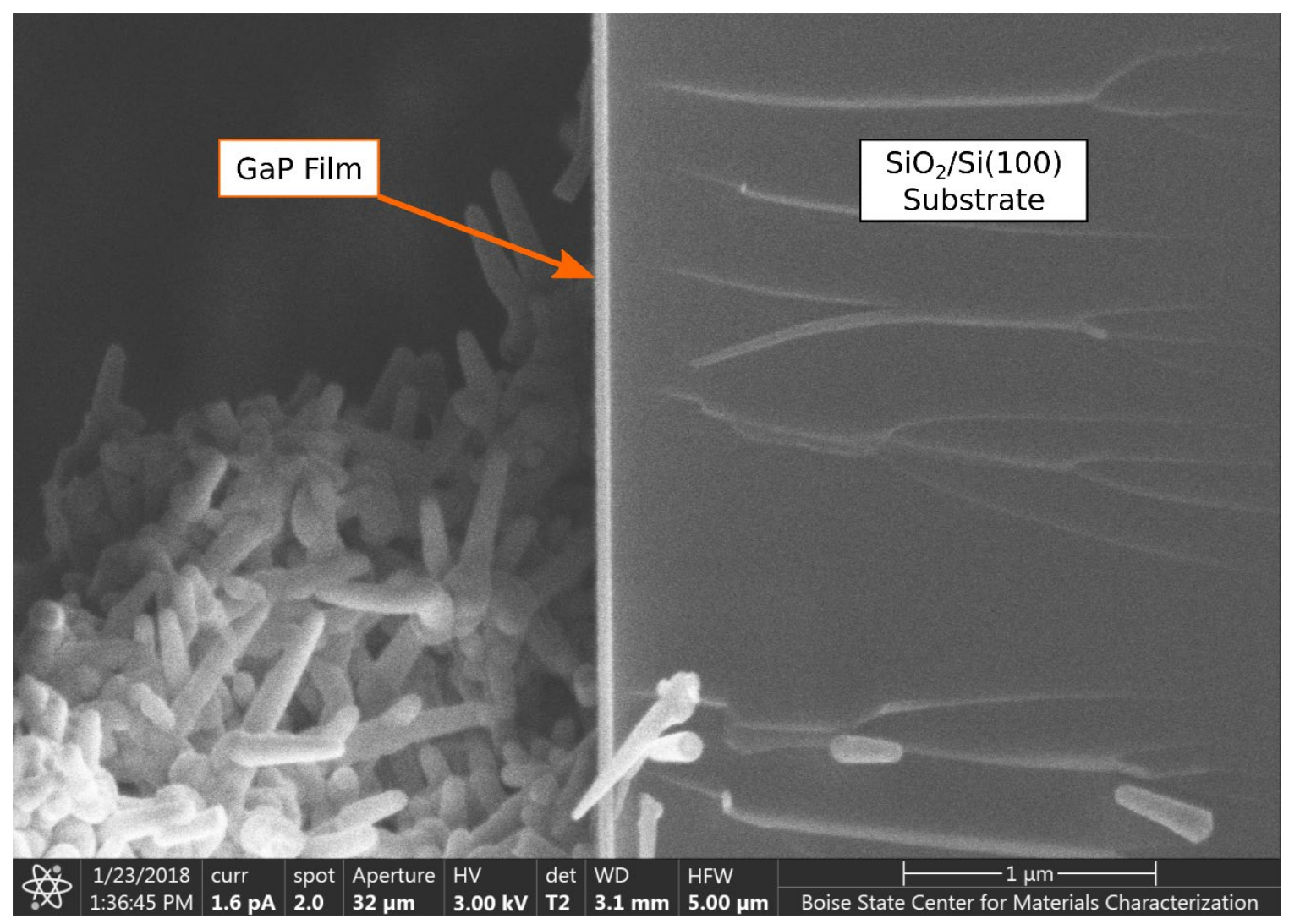

Figure 2.19 Cross-sectional SEM image showing GaP film and a large 3D GaP structure. The sample shown in Figures 2.17 and 2.18 (GaP deposited on $\mathrm{SiO}_{2} / \mathrm{Si}(100)$ at $475^{\circ} \mathrm{C}$ using 150 cycles of TMGa + TDMAP) was scored with a diamond scribe and cleaved prior to cross-sectional imaging. 


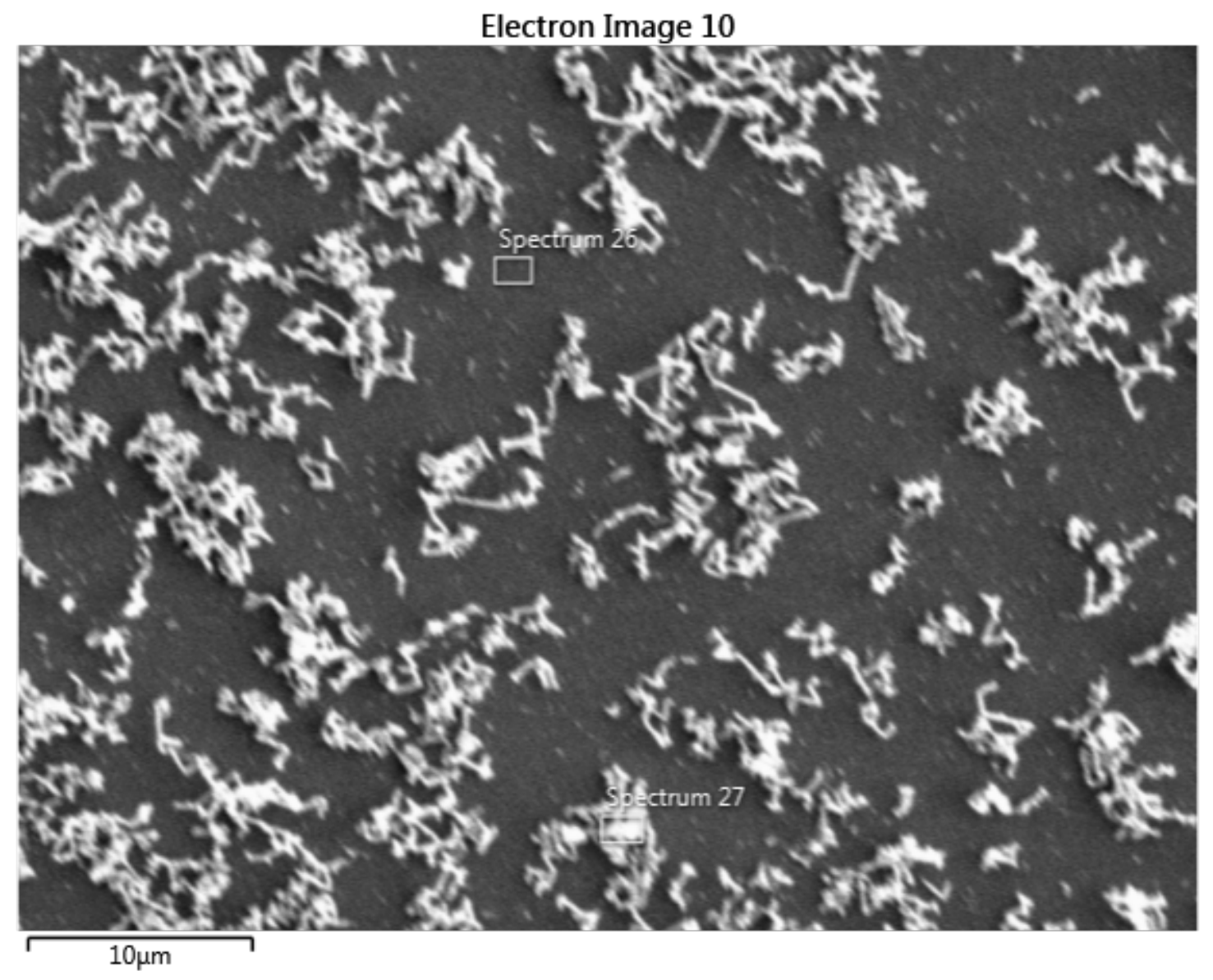

Figure 2.20 $\mathrm{SEM}$ image of $\mathrm{GaP}$ film grown on $\mathrm{SiO}_{2} / \mathrm{Si}(100)$ at $450{ }^{\circ} \mathrm{C}$ using 150 cycles of TMGa + TBP, yielding a many small rod-like GaP structures scattered across the film surface. $\mathrm{SiO}_{2} / \mathrm{Si}(100)$ sample was cleaned in fuming $\mathrm{H}_{2} \mathrm{SO}_{4}$ and then stored in a desiccator for several weeks prior to the deposition experiment, suggesting the need for careful sample preparation with thorough cleaning immediately prior to GaP deposition. 


\section{Electron Image 2}

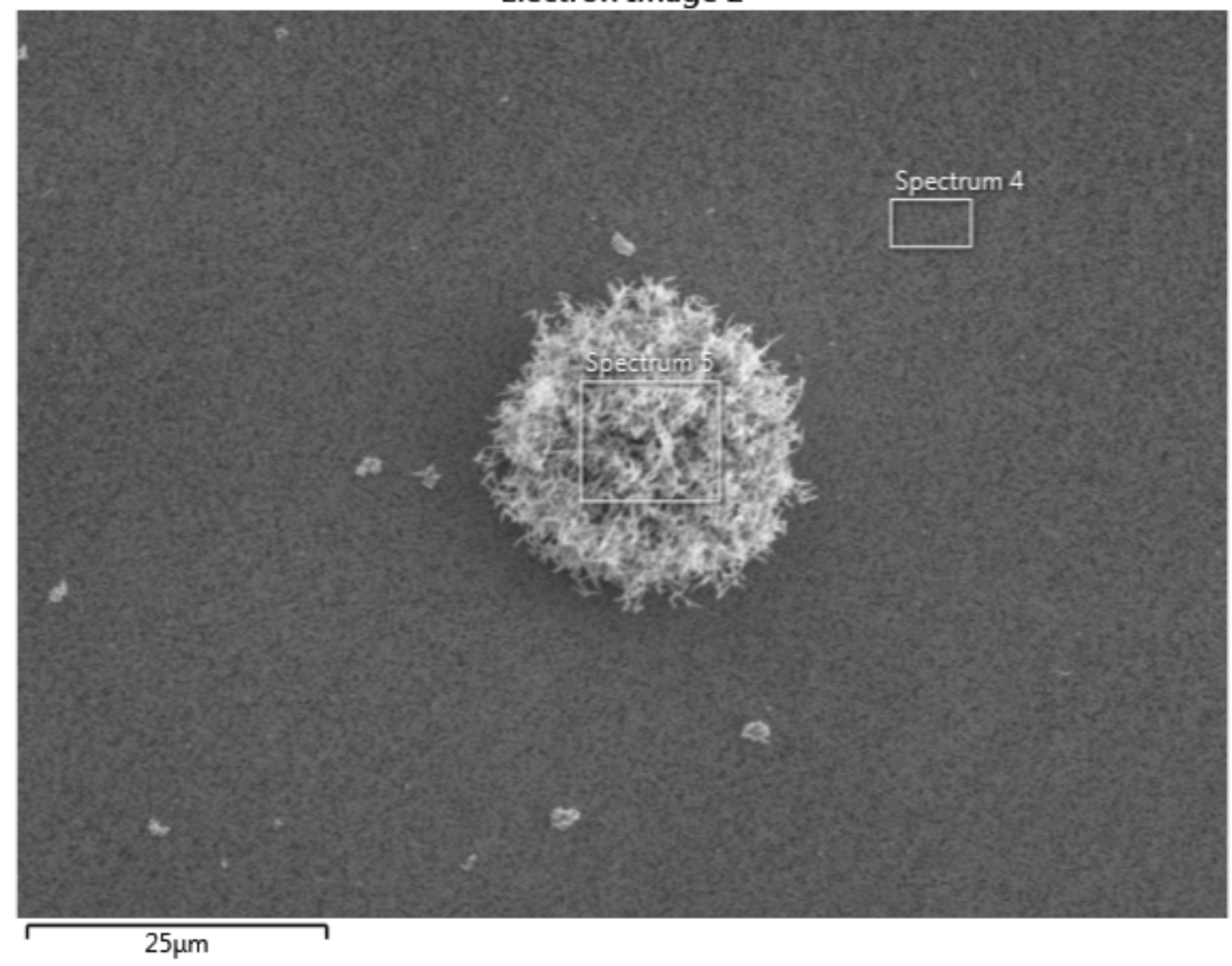

Figure 2.21 $\mathrm{SEM}$ image of GaP film grown on $\mathrm{SiO}_{2} / \mathrm{Si}(100)$ at $450{ }^{\circ} \mathrm{C}$ using 150 cycles of TMGa + TBP, yielding a spherical agglomeration of rod-like GaP structures. $\mathrm{SiO}_{2} / \mathrm{Si}(100)$ sample was cleaned in fuming $\mathrm{H}_{2} \mathrm{SO}_{4}$ and then stored in a desiccator for several weeks prior to the deposition experiment, suggesting the need for careful sample preparation with thorough cleaning immediately prior to GaP deposition. 


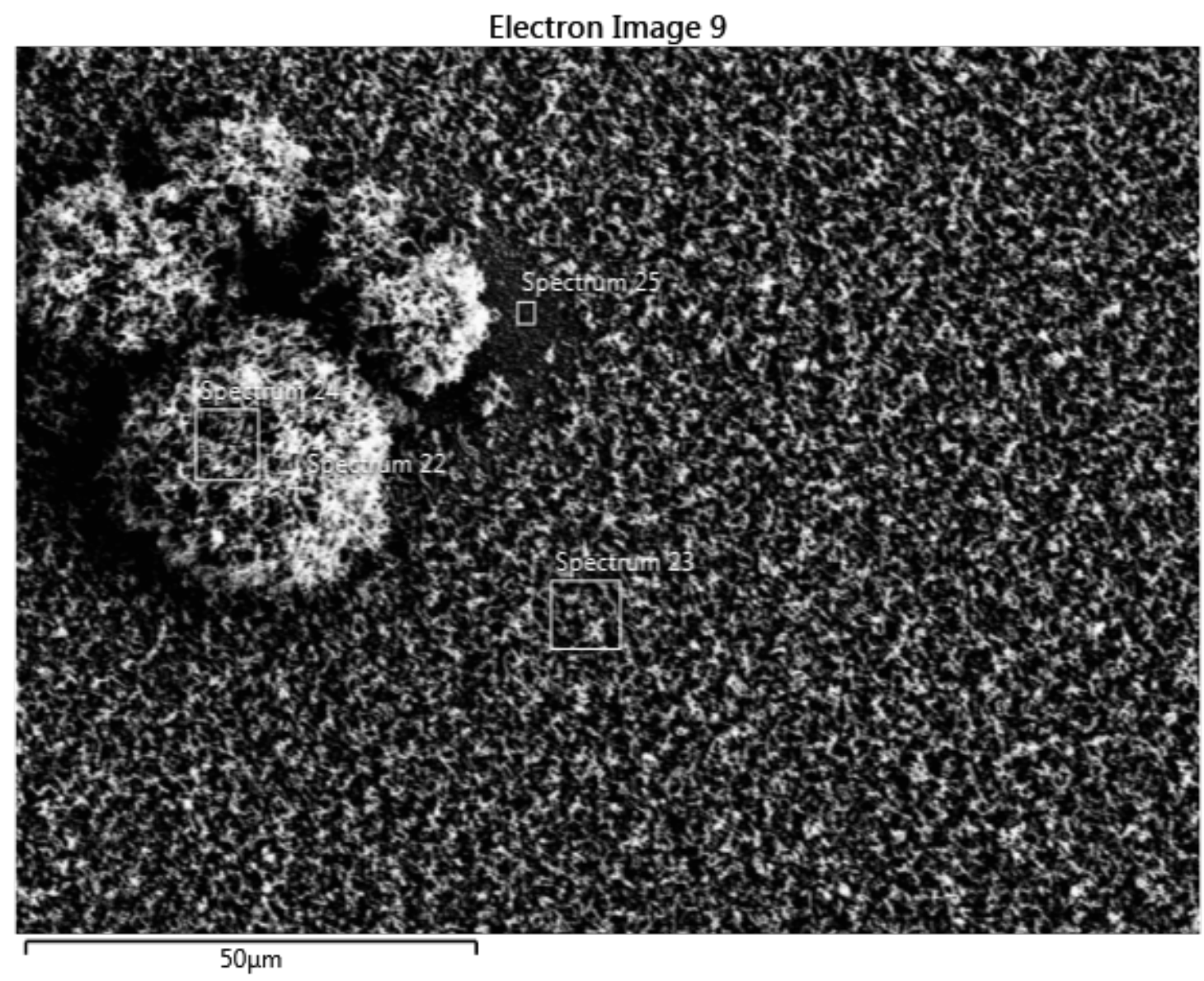

Figure 2.22 $\mathrm{SEM}$ image of $\mathrm{GaP}$ film grown on $\mathrm{SiO}_{2} / \mathrm{Si}(100)$ at $450{ }^{\circ} \mathrm{C}$ using 150 cycles of TMGa + TBP, yielding several spherical agglomerations of rod-like GaP structures atop a carpet-like coating of $\mathrm{GaP}$ rods. $\mathrm{SiO}_{2} / \mathrm{Si}(100)$ sample was cleaned in fuming $\mathrm{H}_{2} \mathrm{SO}_{4}$ and then stored in a desiccator for several weeks prior to the deposition experiment, suggesting the need for careful sample preparation with thorough cleaning immediately prior to GaP deposition.

Equipment malfunctions and spurious leaks to atmosphere further confounded interpretation of GaP deposition results, and the ALD reactor was subsequently redesigned and re-built as described in Section 2.2 to correct the existing problems, including TDMAP condensation and possible CVD-like conditions.

To better understand the various results observed via GaP deposition on substrates, in situ QCM was performed to uncover the reaction mechanisms and variables affecting GaP film growth. QCM experiments utilized a full-flow ALD process due to the 
need for continuous $\mathrm{N}_{2}$ purge to prevent film deposition on the back side of the QCM crystal. Figures 2.23-2.25 show QCM film mass and the computed mass change $(\Delta \mathrm{m})$ of each ALD half cycle for TMGa + TDMAP growth at $450{ }^{\circ} \mathrm{C}$ on ALD-grown $\mathrm{SiO}_{2}$, $\mathrm{Al}_{2} \mathrm{O}_{3}$, and $\mathrm{TiO}_{2}$. Growth on $\mathrm{SiO}_{2}$ and $\mathrm{Al}_{2} \mathrm{O}_{3}$ appear similar with relative low growth per cycle (GPC) values that decrease during the first 50 cycles. Growth on $\mathrm{TiO}_{2}$ shows a significantly larger initial mass gain followed by a higher GPC relative to $\mathrm{SiO}_{2}$ and $\mathrm{Al}_{2} \mathrm{O}_{3}$. The average $\mathrm{GaP} \Delta \mathrm{m}$ values for cycles $41-50$ on $\mathrm{ALD} \mathrm{SiO}_{2}, \mathrm{Al}_{2} \mathrm{O}_{3}$, and $\mathrm{TiO}_{2}$ are $\sim 1.1 \mathrm{ng} / \mathrm{cm}^{2}, \sim 1.7 \mathrm{ng} / \mathrm{cm}^{2}$, and $\sim 2.4 \mathrm{ng} / \mathrm{cm}^{2}$, respectively. It should be noted that the STM-2 LabVIEW library supplied by Inficon outputs film mass in increments of 1 $\mathrm{ng} / \mathrm{cm}^{2}$, so the measured mass changes are on the order of random noise and thermal fluctuations for the timescales shown. Assuming bulk GaP density of $4.14 \mathrm{~g} / \mathrm{cm}^{3}$, these $\Delta \mathrm{m}$ values correspond to GPCs of $\sim 0.03 \AA\left(\mathrm{SiO}_{2}\right), \sim 0.04 \AA\left(\mathrm{Al}_{2} \mathrm{O}_{3}\right)$, and $\sim 0.06 \AA\left(\mathrm{TiO}_{2}\right)$. In all cases, the initial pulse of TMGa produced a large mass gain corresponding to the reaction of TMGa molecules with the hydroxyl groups on the substrate surfaces. With M representing the metal of the oxide, this nucleation reaction can be written as:

$$
\mathrm{M}-\mathrm{OH}+\mathrm{Ga}\left(\mathrm{CH}_{3}\right)_{3} \rightarrow \mathrm{M}-\mathrm{OGa}\left(\mathrm{CH}_{3}\right)_{3-x}+\left(\mathrm{CH}_{4}\right)_{x}
$$

While the above nucleation equation would be similar for each metal oxide, the mass gain for the first $\mathrm{TMGa}$ pulse on $\mathrm{TiO}_{2}$ was significantly larger than on $\mathrm{SiO}_{2}$ or $\mathrm{Al}_{2} \mathrm{O}_{3}$, which may indicate a higher -OH density or decomposition of the TMGa precursor on this surface or some complex surface diffusion processes. TMGa is also known to form dimethylgallium when adsorbed on $\mathrm{Al}_{2} \mathrm{O}_{3}$ and $\mathrm{SiO}_{2}$ surfaces, ${ }^{25,26}$ and was found to have insufficient reactivity with $\mathrm{H}_{2} \mathrm{O}$ for ALD of gallium oxide. ${ }^{27}$ After the first few TMGa + TDMAP cycles on $\mathrm{SiO}_{2}$ and $\mathrm{TiO}_{2}$ at $450{ }^{\circ} \mathrm{C}$, almost no mass changes were observed 
during the TDMAP half-cycle. After the first few TMGa + TDMAP cycles on $\mathrm{Al}_{2} \mathrm{O}_{3}$ at $450{ }^{\circ} \mathrm{C}$, almost no mass changes were observed in the TMGa half-cycle. These results may indicate a process where nearly equal mass is deposited as is released, or they may indicate low reactivity of TMGa with TDMAP. Ultimately, full-flow TMGa + TDMAP GaP ALD nearly stagnated on the oxide surfaces available for crystal pre-coating, indicating poor precursor reactivity unsuitable for full-flow ALD.

Similarly low average $\Delta \mathrm{m}\left(0.8 \mathrm{ng} / \mathrm{cm}^{2}\right)$ and GPC $(0.02 \AA)$ were observed for GaP growth on an $\mathrm{Al}_{2} \mathrm{O}_{3}$-coated resonator using full-flow $\mathrm{TMGa}+\mathrm{TBP}$ at $450{ }^{\circ} \mathrm{C}$. To determine whether greater mass change could be achieved with increased precursor exposure (but without prohibitively long full-flow doses), a handful of quasi-FHP ALD runs were completed with TMGa + TBP with nominal target precursor doses of $\sim 10^{6} \mathrm{~L}$ and $\mathrm{ALD} \mathrm{Al}_{2} \mathrm{O}_{3}$-coated resonators. In this scheme, the chamber was filled to a target pressure with each precursor and all precursor delivery lines were diverted to vacuum. The chamber isolation valve was then closed, and the only gas flux into the chamber was through the QCM back-purge. Hold durations were limited to avoid over-pressurizing the chamber with $\mathrm{N}_{2}$ gas from the QCM back-purge; therefore, target doses of $\sim 10^{7} \mathrm{~L}$ typically used in TMGa + TBP substrate depositions could not be achieved. The QCM data for 100 of these quasi-FHP TMGa + TBP cycles are shown in Figure 2.26. This approach did not reflect the conditions in typical FHP substrate deposition experiments, but it did achieve somewhat larger mass changes relative to full-flow TMGa + TBP cycles. For cycles 91-100 shown in Fig. 2.26, the average calculated $\Delta \mathrm{m}$ and GPC values were $\sim 1.5 \mathrm{ng} / \mathrm{cm}^{2}$ and $\sim 0.4 \AA$, respectively. Though the growth was still quite low, it was approximately double that of the comparable full-flow TMGa + TBP cycles. 


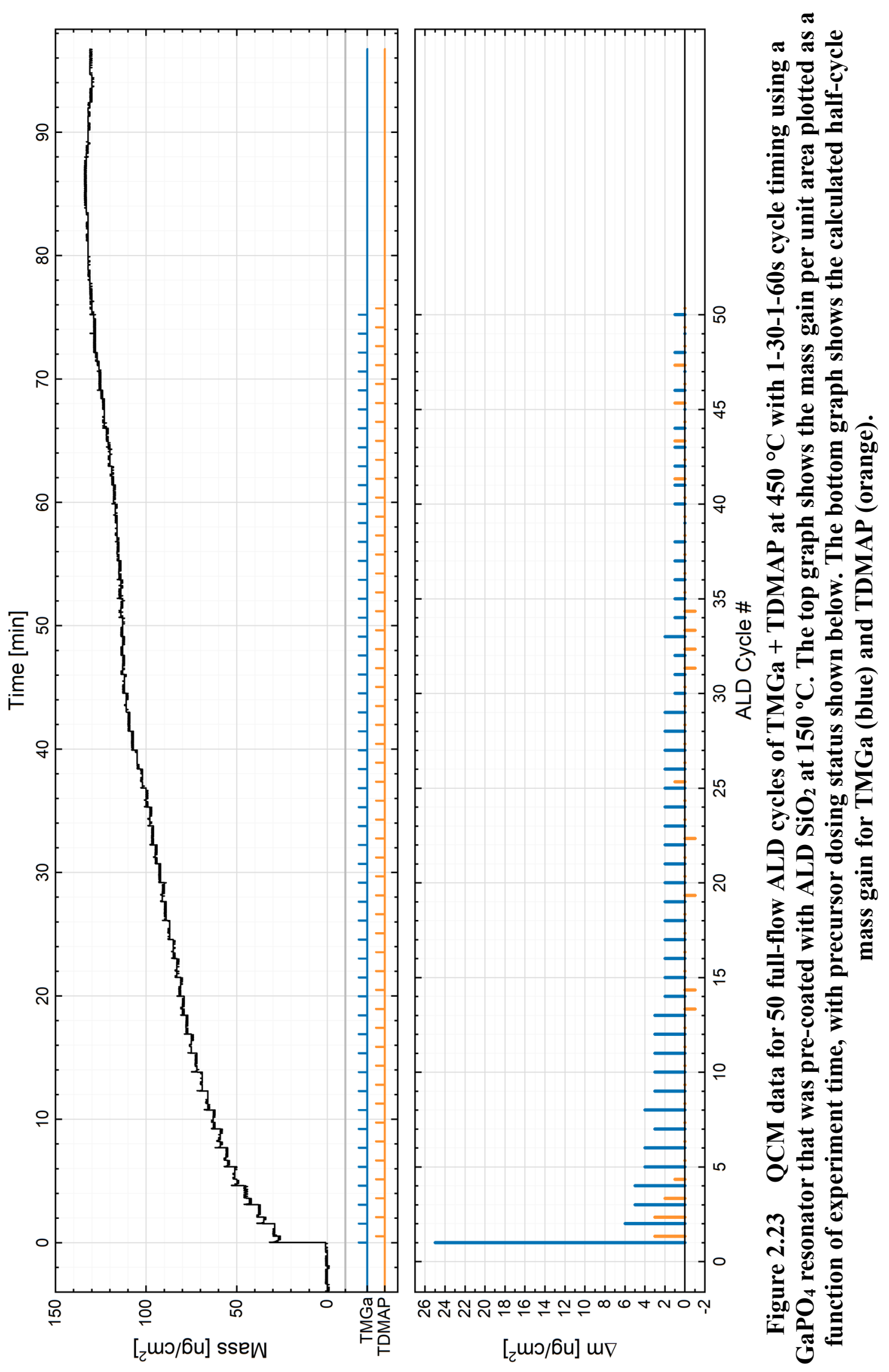



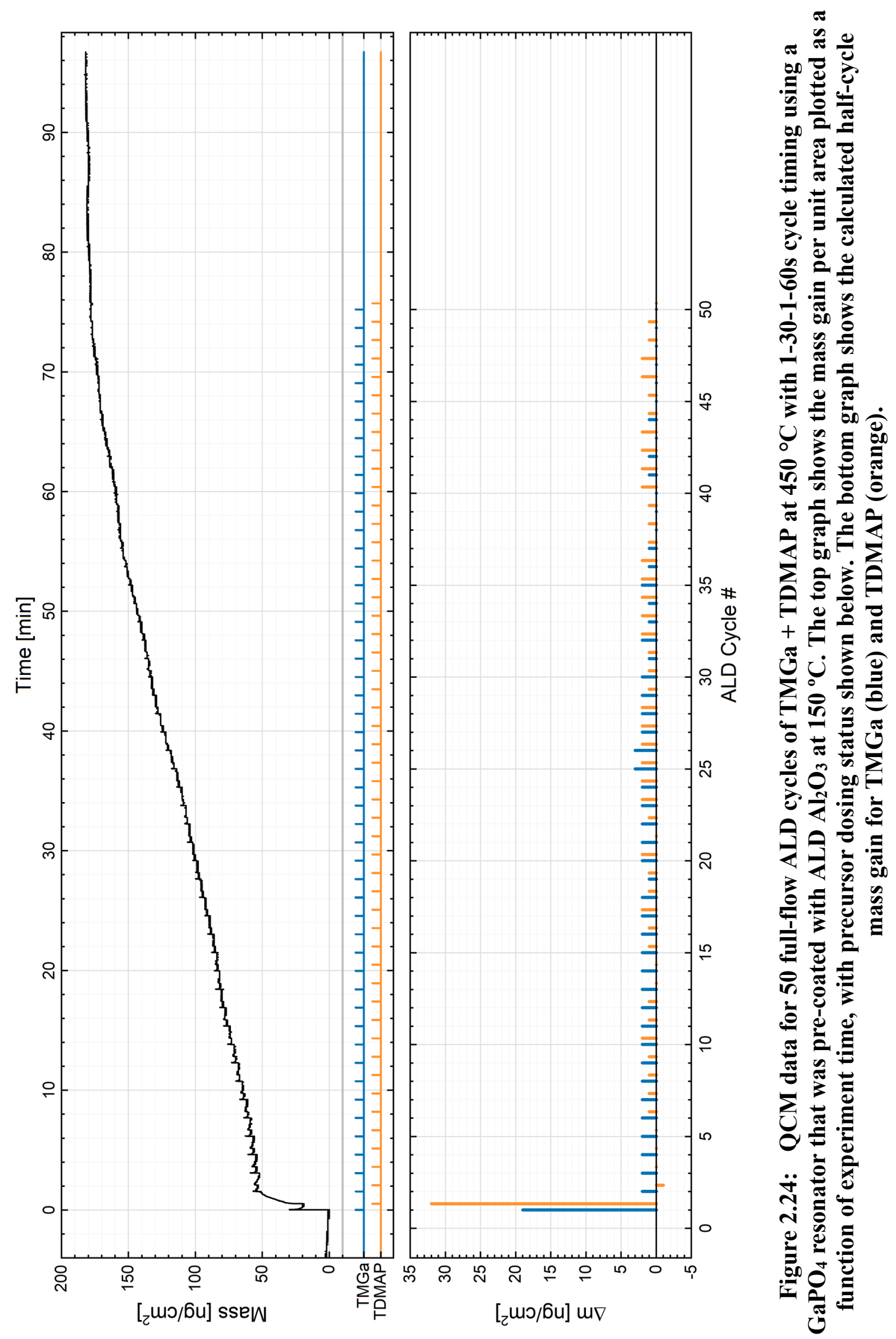


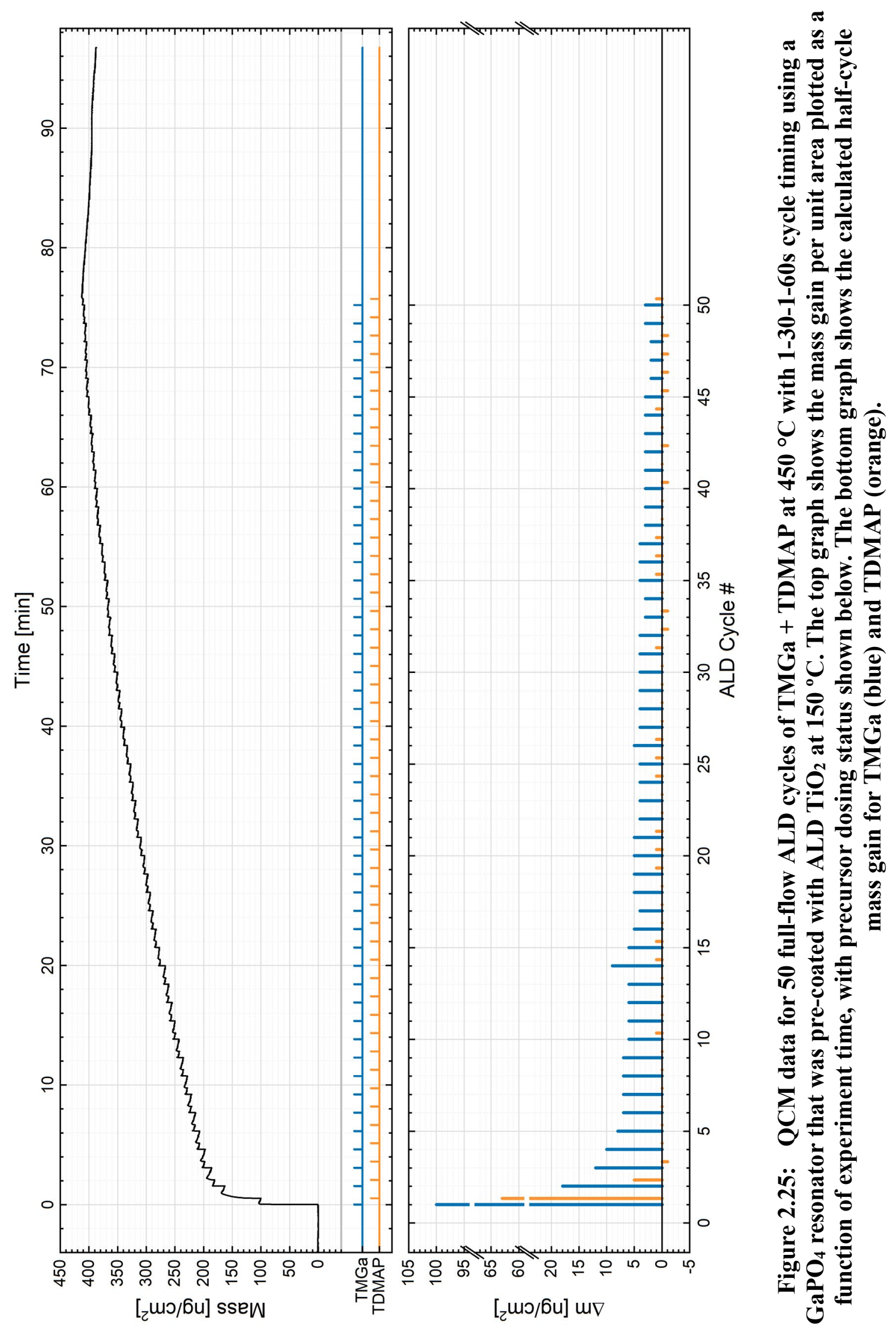




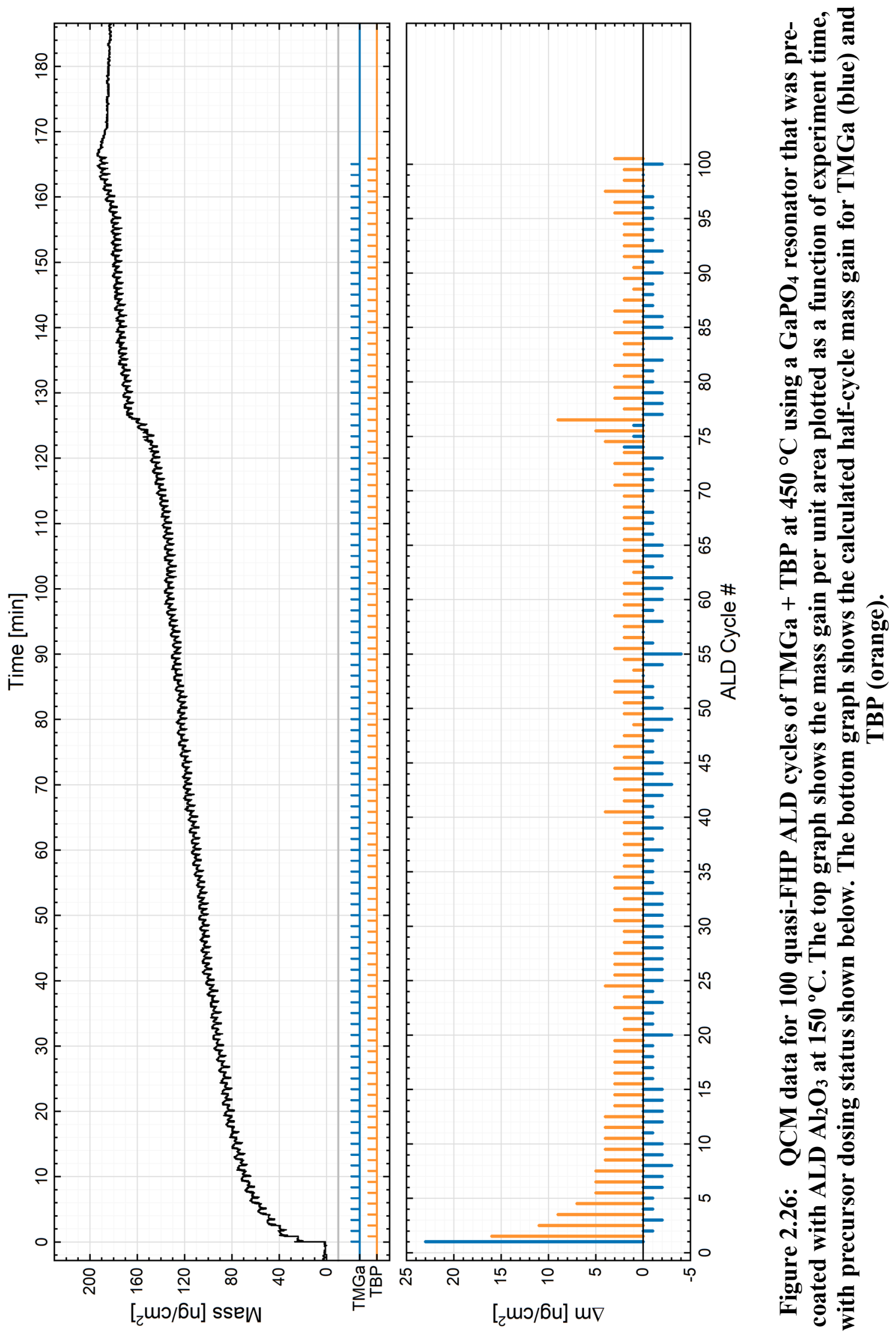




\subsection{Summary and Conclusions}

Although some promising results for ALD of GaP films were observed with both the TMGa + TDMAP and TMGa $+\mathrm{TBP}$ chemistries at $450{ }^{\circ} \mathrm{C}$, reproducibility was quite poor. Both chemistries were highly sensitive to substrate cleanliness and surface preparation, and long precursor exposures during static dosing appeared be necessary to achieve film growth. Additional work is required to elucidate the exact nature of the GaP film growth versus non-ideal 3D growth modes observed. Time-resolved full-scale mass spectrometry using the improved LabVIEW program may prove useful in the investigation of the ALD reaction pathways. Development of a high-temperature QCM sensor compatible with true Fill-Hold-Purge ALD processes may also offer some insight into the results observed via substrate depositions utilizing Fill-Hold-Purge static dosing.

\subsection{References}

1. Akinlami, J. O.; Olatunji, O. A., Optical Properties of Gallium Phosphide (GaP). J. Nat. Sci. Eng. Technol. 2014, 13 (1), 18-27.

2. Graugnard, E.; Chawla, V.; Lorang, D.; Summers, C. J., High filling fraction gallium phosphide inverse opals by atomic layer deposition. Applied Physics Letters 2006, 89 (21), 211102.

3. Graugnard, E.; M. Roche, O.; Dunham, S.; King, J.; N. Sharp, D.; G. Denning, R.; J. Turberfield, A.; Summers, C. J., Replicated Photonic Crystals by Atomic Layer Deposition Within Holographically Defined Polymer Templates. 2009; Vol. 94.

4. $\quad$ Grassman, T. J.; Carlin, J. A.; Galiana, B.; Yang, L. M.; Yang, F.; Mills, M. J.; Ringel, S. A., Nucleation-related defect-free $\mathrm{GaP} / \mathrm{Si}(100)$ heteroepitaxy via metal-organic chemical vapor deposition. Applied Physics Letters 2013, 102 (14), 142102.

5. $\quad$ Emmer, H.; Chen, C. T.; Saive, R.; Friedrich, D.; Horie, Y.; Arbabi, A.; Faraon, A.; Atwater, H. A., Fabrication of Single Crystal Gallium Phosphide Thin Films on Glass. Scientific Reports 2017, 7 (1), 4643.

6. Cambiasso, J.; Grinblat, G.; Li, Y.; Rakovich, A.; Cortés, E.; Maier, S. A., Bridging the Gap between Dielectric Nanophotonics and the Visible Regime with Effectively Lossless Gallium Phosphide Antennas. Nano Letters 2017, 17 (2), 1219-1225.

7. Tilmann, B.; Grinblat, G.; Berté, R.; Özcan, M.; Kunzelmann, V. F.; Nickel, B.; Sharp, I. D.; Cortés, E.; Maier, S. A.; Li, Y., Nanostructured amorphous gallium 
phosphide on silica for nonlinear and ultrafast nanophotonics. Nanoscale Horizons 2020, 5 (11), 1500-1508.

8. $\quad$ Schneider, K.; Baumgartner, Y.; Hönl, S.; Welter, P.; Hahn, H.; Wilson, D. J.; Czornomaz, L.; Seidler, P., Optomechanics with one-dimensional gallium phosphide photonic crystal cavities. Optica 2019, 6 (5), 577-584.

9. Melli, M.; West, M.; Hickman, S.; Dhuey, S.; Lin, D.; Khorasaninejad, M.; Chang, C.; Jolly, S.; Tae, H.; Poliakov, E.; St. Hilaire, P.; Cabrini, S.; Peroz, C.; Klug, M., Gallium phosphide optical metasurfaces for visible light applications. Scientific Reports 2020, 10 (1), 20694.

10. Usui, A.; Sunakawa, H.; Stützler, F. J.; Ishida, K., InGaP/GaAs single quantum well structure growth on GaAs facet walls by chloride atomic layer epitaxy. Applied Physics Letters 1990, 56 (3), 289-291.

11. Yoshimoto, M.; Kajimoto, A.; Matsunami, H., Laser-assisted atomic layer epitaxy of GaP in chemical beam epitaxy. Thin Solid Films 1993, 225 (1), 70-73.

12. McDermott, B. T.; El-Masry, N. A.; Jiang, B. L.; Hyuga, F.; Bedair, S. M.; Duncan, W. M., Ordered GaInP by atomic layer epitaxy. Journal of Crystal Growth 1991, 107 (1), 96-101.

13. McDermott, B. T.; Reid, K. G.; El-Masry, N. A.; Bedair, S. M.; Duncan, W. M.; Yin, X.; Pollak, F. H., Atomic layer epitaxy of GaInP ordered alloy. Applied Physics Letters 1990, 56 (12), 1172-1174.

14. Sakuma, Y.; Ozeki, M.; Ohtsuka, N.; Kodama, K., Comparative study of selflimiting growth of GaAs using different Ga-alkyl compounds: (CH3)3Ga, C2H5(CH3)2Ga, and (C2H5)3Ga. Journal of Applied Physics 1990, 68 (11), 5660-5664.

15. Gong, J. R.; Nakamura, S.; Leonard, M.; Bedair, S. M.; El-Masry, N. A., Low Temperature Growth of AIGaP and GaP on Si Substrates by Atomic Layer Epitaxy. Journal of Electronic Materials 1992, 21 (10), 965-970.

16. Kuninori Kitahara, M. O., and Kazuo Nakajima, Reflection High-Energy Electron Diffraction of Heteroepitaxy in Chemical Vapor Deposition Reactor: Atomic-Layer Epitaxy of GaAs, AlAs and GaP on Si. Japanese Journal of Applied Physics 1993, 32 (3R), 1051.

17. Ishii, M.; Iwai, S.; Kawata, H.; Ueki, T.; Aoyagi, Y., Atomic layer epitaxy of AlP and its application to X-ray multilayer mirror. 1997; Vol. 180, p 15-21.

18. Isshiki, H.; Aoyagi, Y.; Sugano, T., (GaAs)m(GaP)n low dimensional shortperiod superlattice fabricated by atomic layer epitaxy. Microelectronic Engineering 1998, 43-44, 301-307.

19. Ozeki, M.; Haraguchi, T.; Takeuchi, T.; Maeda, K., A comparative study of the growth mechanism of InAs/GaAs and $\mathrm{GaP} / \mathrm{GaAs}$ heterostructures and strained layered superlattices by atomic layer epitaxy. Journal of Crystal Growth 2005, 276 (3), 374-380.

20. Uvarov, A. V.; Gudovskikh, A. S.; Nevedomskiy, V. N.; Baranov, A. I.; Kudryashov, D. A.; Morozov, I. A.; Kleider, J. P., Low temperature epitaxial growth of 
$\mathrm{GaP}$ on Si by atomic-layer deposition with plasma activation. Journal of Physics D: Applied Physics 2020, 53 (34), 345105.

21. Gudovskikh, A. S.; Morozov, I. A.; Uvarov, A. V.; Kudryashov, D. A.; Nikitina, E. V.; Bukatin, A. S.; Nevedomskiy, V. N.; Kleider, J.-P., Low temperature plasma enhanced deposition of GaP films on Si substrate. Journal of Vacuum Science \& Technology A 2017, 36 (2), 021302.

22. Li, N. Y.; Dong, H. K.; Wong, W. S.; Tu, C. W., An evaluation of alternative precursors in chemical beam epitaxy: tris-dimethylaminoarsenic, trisdimethylaminophosphorus, and tertiarybutylphosphine. Journal of Crystal Growth 1996, 164 (1), 112-116.

23. Werbrouck, A.; Shirazi, M.; Mattelaer, F.; Elliott, S. D.; Dendooven, J.; Detavernier, C., A Secondary Reaction Pathway for the Alumina Atomic Layer Deposition Process with Trimethylaluminum and Water, Revealed by Full-Range, TimeResolved In Situ Mass Spectrometry. The Journal of Physical Chemistry C 2020, 124 (48), 26443-26454.

24. Burton, B. B.; Boleslawski, M. P.; Desombre, A. T.; George, S. M., Rapid $\mathrm{SiO}_{2}$ Atomic Layer Deposition Using Tris(tert-pentoxy)silanol. Chemistry of Materials 2008, 20 (22), 7031-7043.

25. Morrow, B. A.; McFarlane, R. A., Trimethylgallium adsorbed on silica and its reaction with phosphine, arsine, and hydrogen chloride: an infrared and Raman study. The Journal of Physical Chemistry 1986, 90 (14), 3192-3197.

26. McFarlane, R. A.; Morrow, B. A., An infrared study of trimethylgallium adsorbed on alumina and the Lewis/hydroxyl site ratio. The Journal of Physical Chemistry 1988, $92(20), 5800-5803$.

27. Comstock, D. J.; Elam, J. W., Atomic Layer Deposition of $\mathrm{Ga}_{2} \mathrm{O}_{3}$ Films Using Trimethylgallium and Ozone. Chemistry of Materials 2012, 24 (21), 4011-4018. 


\section{CHAPTER THREE: ATOMIC LAYER DEPOSITION OF SODIUM FLUORIDE THIN} FILMS

This chapter was published in Journal of Vacuum Science \& Technology A as an Editor's Pick. Reproduced from Journal of Vacuum Science \& Technology A 39, 032405 (2021) with permission of AIP publishing.

https://doi.org/10.1116/6.0000847

*This chapter contains minor modifications from the published text.

Sara Kuraitis ${ }^{1}$, Donghyeon Kang ${ }^{2}$, Anil U. Mane ${ }^{2}$, Hua Zhou ${ }^{3}$, Jake Soares ${ }^{1}$, Jeffrey W. Elam $^{2}$, Elton Graugnard ${ }^{1}$

${ }^{1}$ Micron School of Materials Science \& Engineering, Boise State University, 1910 University Dr., Boise, Idaho 83725

${ }^{2}$ Applied Materials Division, Argonne National Laboratory, 9700 S. Cass Ave, Argonne, Illinois 60439

${ }^{3}$ X-ray Science Division, Advanced Photon Source, Argonne National Laboratory, Lemont, IL 60439 


\subsection{Abstract}

The need for advanced energy conversion and storage devices remains a critical challenge amid the growing worldwide demand for renewable energy. Metal fluoride thin films are of great interest for applications in lithium-ion and emerging rechargeable battery technologies, particularly for enhancing the stability of the electrode-electrolyte interface and thereby extending battery cyclability and lifetime. Reported within, sodium fluoride $(\mathrm{NaF})$ thin films were synthesized via atomic layer deposition (ALD). NaF growth experiments were carried out at reactor temperatures between 175 and $250{ }^{\circ} \mathrm{C}$ using sodium tert-butoxide and HF-pyridine solution. The optimal deposition temperature range was $175-200{ }^{\circ} \mathrm{C}$, and the resulting $\mathrm{NaF}$ films exhibited low roughness $\left(\mathrm{R}_{\mathrm{q}} \approx 1.6 \mathrm{~nm}\right.$ for films of $\sim 8.5 \mathrm{~nm})$, nearly stoichiometric composition $(\mathrm{Na}: \mathrm{F}=1: 1.05)$, and a growth per cycle value of $0.85 \AA$ /cycle on $\mathrm{SiO}_{2}$ substrates. These results are encouraging for future applications of $\mathrm{NaF}$ thin films in the development of improved energy capture and storage technologies.

\subsection{Introduction}

In the decades since their introduction and commercialization, lithium-ion batteries (LIBs) have dominated the rechargeable battery market. However, commercial LIBs suffer from high cost due to the limited availability of lithium resources across the world. ${ }^{1}$ Sodium-ion batteries (SIBs) are among the emerging battery technologies viewed as promising alternatives to LIBs. Unlike lithium, sodium resources are widespread and include abundant supply in the world's oceans. SIBs are therefore expected to be relatively low cost and more environmentally friendly than current commercial LIBs. 
SIBs operate on the same fundamental principles as LIBs, so they also experience many of the same problems, such as limited theoretical energy density, structural instability of anode/cathode materials, dendrite formation, and short cycle life. Significant progress has been made towards overcoming these challenges through concentrated research in LIBs, and similar approaches are being explored in the continued development of SIBs. ${ }^{2-6}$ In particular, control over the electrolyte interfacesboth solid electrolyte interphase (SEI) layers at the anode and cathode-electrolyte interface (CEI) — is a key to improving battery performance and stability. In a common approach ${ }^{7}$, ultra-thin layer coatings are introduced to form stable, ion-conductive interfaces between the electrolyte and the electrodes. Recently, atomic layer deposition (ALD) has been recognized as a promising method to deposit thin film electrode coatings, which must be pinhole-free in order to form stable interfaces. ${ }^{6,111}$ ALD is a deposition technique that enables conformal coating of complex substrates with subnanometer thickness control. ${ }^{12,13}$ ALD utilizes cyclic self-limiting surface reactions of alternating doses of chemical precursor vapors that react with a substrate surface to form a thin film. Because ALD is self-limiting and conformal across complex substrate geometries, it is particularly well-suited for depositing artificial interface layers in emerging battery technologies, including three-dimensional nano-ribbon and carbon nanotube sponge electrodes. ${ }^{6,8-10}$

Metal fluorides are one class of materials being explored for advanced battery applications, and have demonstrated promising results as both electrodes and solid electrolytes in LIBs. ${ }^{14-17}$ Sodium fluoride $(\mathrm{NaF})$ has shown utility in SIB applications as a cathode constituent material and as a solid-electrolyte interface layer on sodium metal 
anodes. ${ }^{18,19} \mathrm{NaF}$ films have been deposited via CVD, ${ }^{20-22}$ as well as sputtering and spincoating for use in solar cells. ${ }^{23-26}$ All of these deposition methods lack the conformality and sub-nanometer-level precision of ALD, and although ALD has been used in the synthesis of many metal fluorides, no such process has been reported for ALD of NaF.

In this work, we report the synthesis of $\mathrm{NaF}$ thin films via ALD using sodium tert-butoxide $\left(\mathrm{NaO}^{t} \mathrm{Bu}\right)$ and $\mathrm{HF}$-pyridine solution through the process illustrated in Figure 3.1. $\mathrm{NaO}^{t} \mathrm{Bu}$ is an air-sensitive solid that has previously been used for ALD of sodiumoxide-containing materials, ${ }^{27-30}$ and HF-pyridine solution has been used for ALD of many metal fluorides including lithium fluoride using lithium tert-butoxide. ${ }^{16,17}$ In situ process characterization with a quartz crystal microbalance (QCM) was utilized to establish process parameters for self-limiting surface chemistry. For ex situ film characterization, $\mathrm{NaF}$ films were deposited on $\mathrm{Si}(100)$ coupons terminated with a native oxide layer $(\sim 20$ Å). Films were characterized using X-ray photoelectron spectroscopy (XPS), spectroscopic ellipsometry (SE), grazing incidence X-ray diffraction (GIXRD), atomic force microscopy (AFM), and scanning electron microscopy (SEM). 


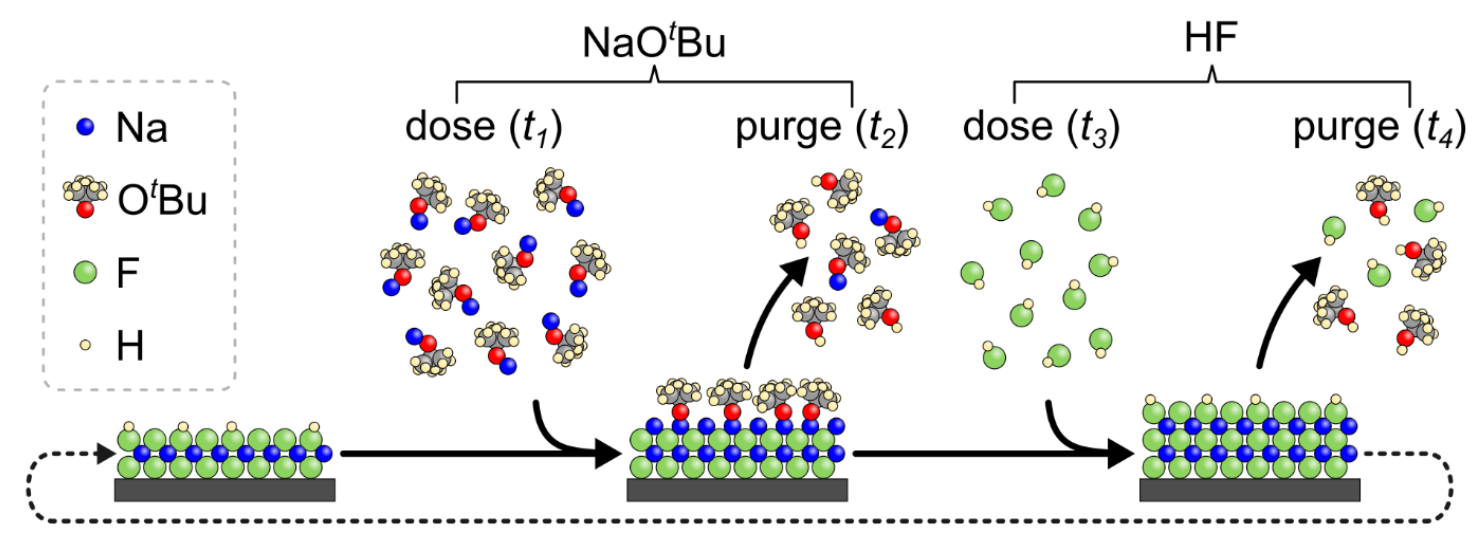

Figure 3.1 Illustration of the ALD process for $\mathrm{NaF}$ using $\mathrm{NaO}^{t} \mathrm{Bu}$ and $\mathrm{HF}$ pyridine solution. Pyridine is not included in the schematic because it does not participate in the ALD surface reactions. The ALD cycle consists of four steps, each defined by a corresponding step time $t_{\mathrm{i}}$ : 1) $\mathrm{NaO}^{t} \mathrm{Bu}$ dose, 2) purge, 3) HF-pyridine dose, 4) purge. Temporal separation of the precursor doses ensures that reactions occur only at the substrate surface (not in the vapor phase), and ultimate film thickness is therefore determined by the number of $\mathrm{NaO}^{t} \mathrm{Bu}+\mathrm{HF}$-pyridine cycles completed.

\subsection{Experimental}

\subsubsection{NaF Deposition}

ALD was performed in a custom-built viscous flow reactor attached to an argonfilled glove box. ${ }^{31}$ The argon-filled glove box was used to prevent the hygroscopic $\mathrm{NaF}$ from absorbing atmospheric $\mathrm{H}_{2} \mathrm{O}$ vapor, which might change the properties of the films. The process was controlled and monitored using custom LabVIEW software. The reaction chamber - a $4.76 \mathrm{~cm}$ diameter stainless steel tube-was heated to $200{ }^{\circ} \mathrm{C}$ and was maintained at $\sim 1$ Torr internal pressure with $270 \mathrm{sccm}$ flow of ultra-high purity argon carrier gas $(99.999 \%$ Ar). A subset of experiments was also performed at temperatures of $175^{\circ} \mathrm{C}, 225^{\circ} \mathrm{C}$, and $250^{\circ} \mathrm{C}$ to explore the temperature dependence of the ALD process. ALD experiments below a growth temperature of $175^{\circ} \mathrm{C}$ were not attempted to avoid condensation of the $\mathrm{NaO}^{t} \mathrm{Bu}$ compound. $\mathrm{NaO}^{t} \mathrm{Bu}(97 \%$ purity, SigmaAldrich) is a white, crystalline powder with a melting point of $180{ }^{\circ} \mathrm{C}$ that adopts a 
hexamer structure and has a vapor pressure of 0.75 Torr at $140{ }^{\circ} \mathrm{C} .{ }^{30}$ The $\mathrm{NaO}^{t} \mathrm{Bu}$ was contained in a custom-machined stainless-steel bubbler and heated to $130-140{ }^{\circ} \mathrm{C}$ bottom-of-pot temperature. $\mathrm{NaO}^{t} \mathrm{Bu}$ was delivered to the reaction chamber by diverting $45 \mathrm{sccm}$ Ar through the bubbler during each dose. HF-pyridine ( 70\% HF, $\sim 30 \%$ pyridine, Sigma-Aldrich) was contained in an unheated stainless-steel cylinder (Swagelok). The NaF ALD cycle is defined by the $\mathrm{NaO}^{t} \mathrm{Bu}$ dose time $\left(t_{1}\right)$ and purge time $\left(t_{2}\right)$, as well as the HF-pyridine dose time $\left(t_{3}\right)$ and purge time $\left(t_{4}\right)$, with the overall cycle timing denoted $t_{1}-t_{2}-t_{3}-t_{4}$. Although a range of dose and purge times were explored for both precursors, typical values were $3-20-2-15$, where each time is measured in seconds.

\subsubsection{Characterization}

In situ quartz crystal microbalance (QCM) measurements were performed using a welded ALD sensor head (Inficon) with a $6 \mathrm{MHz}$ RC-cut quartz crystal (Phillip Technologies). To minimize film deposition on the back side of the crystal, the sensor head was continually purged with Ar gas to bring the total chamber pressure up to $\sim 1.1$ Torr. The QCM was brought to thermal equilibrium in the reaction chamber over several hours, and the crystal was coated with ALD alumina $\left(\mathrm{Al}_{2} \mathrm{O}_{3}\right)$ using alternating cycles of trimethylaluminum and $\mathrm{H}_{2} \mathrm{O}$ prior to each $\mathrm{NaF}$ deposition to prepare a well-defined starting surface for the NaF ALD. Frequency shifts due to film deposition were measured with an STM-2 Thin Film Rate/Thickness Monitor (Inficon). Mass changes per unit area $(\Delta \mathrm{m})$ were calculated according to the Sauerbrey equation, as output by the STM-2 LabVIEW library supplied by Inficon.

For ex situ characterization, 100 ALD cycles of NaF were performed on $\mathrm{Si}(100)$ with $\sim 20 \AA$ native oxide (West Coast Silicon). To minimize air exposure prior to 
characterization, $\mathrm{NaF}$-coated samples were stored under argon inside the reactor-attached glove box or in mylar bags heat-sealed inside of the glove box.

X-ray photoelectron spectrometry (XPS) measurements were performed on a Thermo Scientific K-Alpha XPS system operating in standard lens mode. The X-ray source was $\mathrm{Al} \mathrm{K \alpha}$ with a spot size of $400 \mu \mathrm{m}$. Survey scans used a pass energy of 200.0 $\mathrm{eV}$ and step size of $1.000 \mathrm{eV}$, while high-resolution scans used a pass energy of $50.0 \mathrm{eV}$ and step size of $0.100 \mathrm{eV}$. Five scans were averaged for each sample. The XPS data were analyzed using Thermo Scientific Avantage software, and all spectra were referenced to the adventitious $\mathrm{C}$ 1s peak $(284.8 \mathrm{eV})$. Additional details on XPS analysis are provided in Appendix B.2.

Spectroscopic ellipsometry (SE) was used to determine film thickness. For NaF deposition on $\mathrm{Si}$, measurements were performed on a J.A. Woollam alpha-SE ellipsometer, and data were collected in standard acquisition mode with a single scan at an incidence angle of $70.094^{\circ}$ at 188 wavelength increments from $380-900 \mathrm{~nm}$. For NaF deposition on $\mathrm{Al}_{2} \mathrm{O}_{3}$ coated $\mathrm{Si}$, measurements were performed on a J.A. Woollam M2000 ellipsometer, and data were collected at multiple angles with 328 wavelength steps from $380-900 \mathrm{~nm}$. Analysis was performed using CompleteEASE 5.1 software. The thicknesses of the native oxide layer (20.06 $\AA$ ) and alumina layer (542 $\AA$ ) were measured prior to $\mathrm{NaF}$ deposition, and the thickness of the deposited $\mathrm{NaF}$ layer was fit with a $\mathrm{NaF}$ Sellmeier model for bulk NaF provided in the material library of the CompleteEASE software.

Grazing incidence X-ray diffraction (GIXRD) experiments were performed at an undulator beamline 33-ID-D at the Advanced Photon Source (APS), Argonne National 
Laboratory on a six-circle Kappa goniometer with an X-ray energy of $10.5 \mathrm{keV}$ using a pixel array area detector (Dectris Pilatus 100K). The incoming X-ray beam had a flux of $10^{12}$ photons per second. To optimize the thin film diffraction signal, a small angle of incidence near the substrate total reflection critical angle (e.g., $0.2-0.3^{\circ}$ ) was adopted for the GIXRD scans. NaF thin film samples were placed in a He flow cell during ex situ synchrotron GIXRD measurements to minimize air exposure.

Atomic force microscopy (AFM) images of $\mathrm{NaF}$ surfaces were obtained on Bruker Dimension FastScan AFMs equipped with Nanoscope V Controllers operating in peak-force tapping mode using ScanAsyst-Air-HR probes. As-deposited samples were stored in Ar and transferred in an Ar-filled vessel to an AFM housed in an Ar-filled MBraun3-glove glovebox. Additional air-exposed samples were removed from the Ar storage environment and imaged in ambient conditions after $\sim 30$ min of air exposure. AFM images were processed with Gwyddion $2.56 .{ }^{32}$ Prior to root mean square roughness calculations, AFM images were leveled with a mean plane subtraction and row alignment (median subtraction) to remove scan line artifacts.

Scanning electron microscopy (SEM) was performed on an FEI Teneo FESEM using an in-column secondary electron detector (accelerating voltage $=1.00 \mathrm{kV}$, aperture $=32 \mu \mathrm{m}$, working distance $=2.0 \mathrm{~mm}$ ). Samples were stored in $\mathrm{Ar}$ and transported in an Ar-filled vessel before undergoing brief air exposure while being transferred into the SEM chamber.

\subsection{Results and Discussion}

In situ QCM was used to establish precursor dose and purge times, and to examine steady-state growth behavior of the $\mathrm{NaO}^{t} \mathrm{Bu}+\mathrm{HF}$-pyridine ALD chemistry. 
Steady-state growth for typical cycle timing of $3-20-2-15$ at $200{ }^{\circ} \mathrm{C}$ is shown in Figure 2a, with two cycles shown in detail in Figure 3.2b.
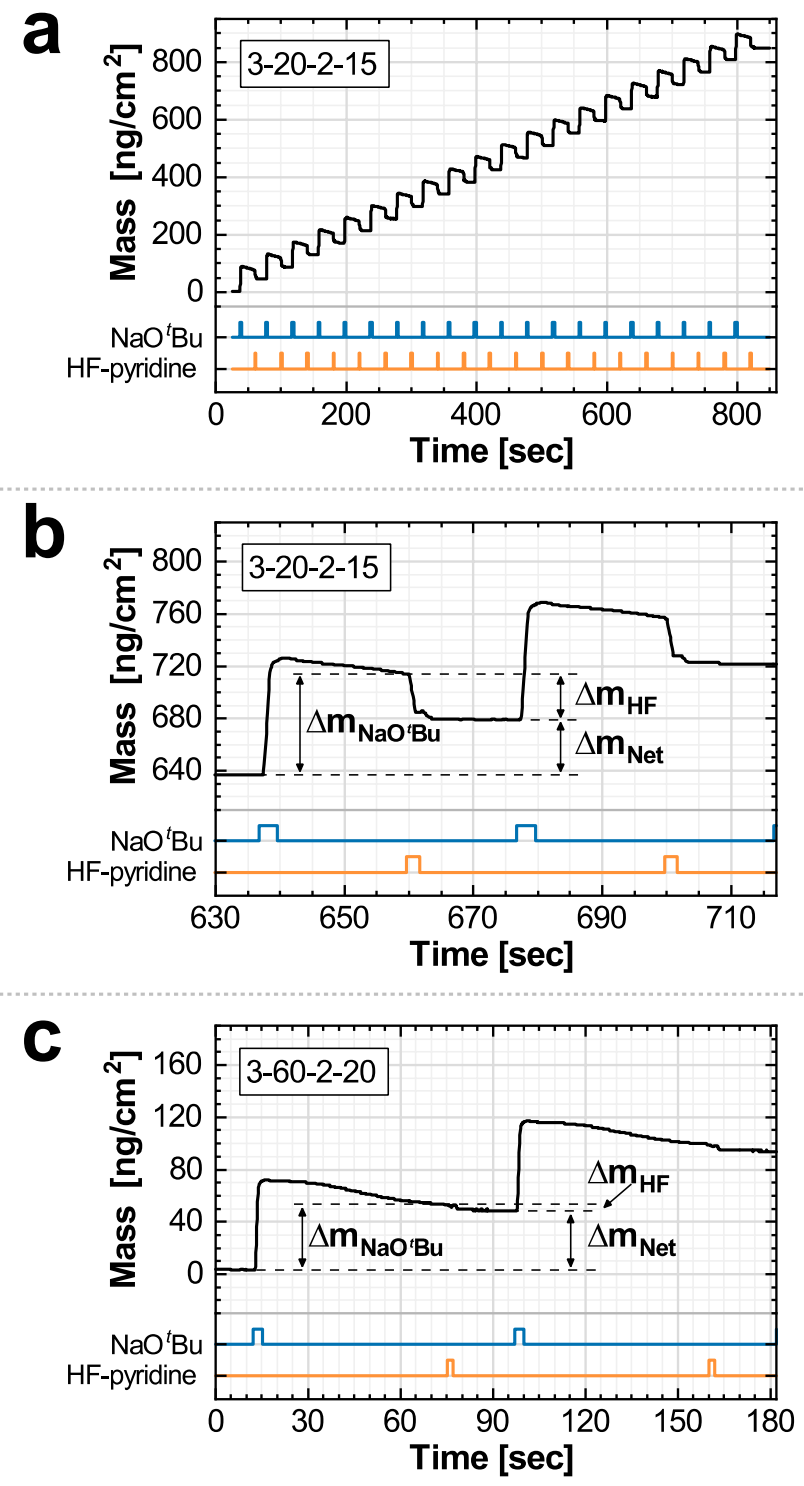

Figure 3.2 QCM data for (a) steady-state NaF growth with 3-20-2-15 cycle timing, (b) detailed view of two cycles with 3-20-2-15 cycle timing, and (c) detailed view of two cycles with 3-60-2-20 cycle timing. The $x$ - and $y$-scale values have been shifted so that both start at zero for the graphed data, though additional $\mathrm{NaF}$ cycles were completed prior those shown (in the case of Fig. 3.2a, 39 cycles were completed prior to the steady-state cycles shown). All QCM measurements were performed at $200{ }^{\circ} \mathrm{C}$ and normalized to QCM of alumina (trimethylaluminum + water) to correct for any back-side deposition on the QCM crystal. 
For the last 15 ALD cycles shown in Fig. 3.2a (3-20-2-15 cycle timing), the $\mathrm{NaO}^{t} \mathrm{Bu}$ half-cycle yielded an average mass change per unit area of $\Delta \mathrm{m}_{\mathrm{NaO} t \mathrm{Bu}}=78 \mathrm{ng} / \mathrm{cm}^{2}$ ( std. dev. $=0.2 \mathrm{ng} / \mathrm{cm}^{2}$ ), while the HF-pyridine half-cycle yielded a mass change of $\Delta \mathrm{m}_{\mathrm{HF}}$ $=-35 \mathrm{ng} / \mathrm{cm}^{2}\left(\mathrm{std} . \mathrm{dev} .=0.1 \mathrm{ng} / \mathrm{cm}^{2}\right)$. The average mass change for a complete ALD cycle was $\Delta \mathrm{m}_{\mathrm{Net}}=42 \mathrm{ng} / \mathrm{cm}^{2}$ (std. dev. $\left.=0.02 \mathrm{ng} / \mathrm{cm}^{2}\right)$. Assuming an upper bound film density of $\rho_{\text {film }}=2.56 \mathrm{~g} / \mathrm{cm}^{3}$ for bulk crystalline $\mathrm{NaF}$, the expected thickness change can be calculated using $\rho_{\text {film }}=\Delta \mathrm{m}_{\text {cycle }} / \Delta \mathrm{t}_{\text {cycle, }}$, to be $\Delta \mathrm{t}_{\text {cycle }} \geq 1.7 \AA /$ cycle. Two cycles of $3-$ 60-2-20 are shown in Figure 3.2c, illustrating markedly different mass change trends compared to Figures 3.2a,b. The initial mass gain from the $\mathrm{NaO}^{t} \mathrm{Bu}$ dose was smaller and some mass was subsequently lost during the prolonged purge $\left(\Delta \mathrm{m}_{\mathrm{NaOtBu}} \approx 50 \mathrm{ng} / \mathrm{cm}^{2}\right)$, and the HF-pyridine dose yielded relatively little mass change $\left(\Delta \mathrm{m}_{\mathrm{HF}} \approx-5 \mathrm{ng} / \mathrm{cm}^{2}\right)$. Interestingly, the net mass change for a complete ALD cycle remained similar $\left(\Delta \mathrm{m}_{\mathrm{Net}} \approx\right.$ $45 \mathrm{ng} / \mathrm{cm}^{2}$ ) despite the large change in the behavior of each half-cycle. As a variety of dose and purge times were explored, increased purge times following $\mathrm{NaO}^{t} \mathrm{Bu}$ doses were observed to cause a distinct change in process behavior. QCM data for additional cycle timing variations is provided in Appendix B.1 Fig. B1. As shown in Fig. B3, microdosing experiments revealed that $\mathrm{NaO}^{t} \mathrm{Bu}$ doses were not self-limiting for pulse-purge times of $3-60$. This phenomenon associated with prolonged $\mathrm{NaO}^{t} \mathrm{Bu}$ purge time was further investigated by depositing 100 ALD cycles of $\mathrm{NaF}$ on silicon substrates to determine whether the observed differences in mass change behavior would correlate with differences in film properties. Film thickness and composition were nearly identical for films deposited at $200{ }^{\circ} \mathrm{C}$ with a variety of cycle timing variations (see XPS composition results in Appendix B.2 Figs. B5, B8-B9 and spectroscopic ellipsometry 
thickness results in Figs. B12, B14). We postulate that the mass loss during prolonged purges following each $\mathrm{NaO}^{t} \mathrm{Bu}$ dose was due to the relatively slow decomposition and loss of tert-butoxide ligands, leaving dangling bonds on the surface that were able to react with subsequent repeated $\mathrm{NaO}^{t} \mathrm{Bu}$ doses. However, following up with an $\mathrm{HF}-$ pyridine dose after the prolonged purge re-saturated the surface and yielded nearly identical net mass change and film properties, so this instability of the tert-butoxide ligands may be inconsequential for binary ALD of NaF at $200{ }^{\circ} \mathrm{C}$.

We propose the following half-reactions of NaF ALD, analogous to those previously proposed for similar LiF ALD chemistry: ${ }^{17}$

$$
\begin{aligned}
& -(\mathrm{H})_{x}{ }^{*}+\mathrm{NaO}^{t} \mathrm{Bu} \rightarrow \mathrm{Na}\left(\mathrm{O}^{t} \mathrm{Bu}\right)_{1-x}{ }^{*}+x \mathrm{HO}^{t} \mathrm{Bu} \\
& \mathrm{Na}\left(\mathrm{O}^{t} \mathrm{Bu}\right)_{1-x}{ }^{*}+\mathrm{HF} \rightarrow \mathrm{NaF}-(\mathrm{H})_{x}{ }^{*}+(1-x) \mathrm{HO}^{t} \mathrm{Bu}
\end{aligned}
$$

where asterisks denote surface species. Defining $\mathrm{R}$ as the ratio between $\Delta \mathrm{m}_{\mathrm{NaO} t \mathrm{Bu}}$ and $\Delta \mathrm{m}_{\mathrm{Net}}$ for the $3-20-2-15$ cycle timing:

$$
\mathrm{R}=\Delta \mathrm{m}_{\mathrm{NaO} t \mathrm{Bu}} / \Delta \mathrm{m}_{\mathrm{Net}}=1.8
$$

This ratio can also be expressed in terms of the atomic weights of the species in Eqs. 1-2:

$$
\mathrm{R}=[73(1-x)+23-x] / 42
$$

Solving for $x$ from Eqs. 3-4 yields $x=0.26$, indicating an average of $26 \%$ of the tertbutoxide ligands were removed as tert-butanol during the $\mathrm{NaO}^{t} \mathrm{Bu}$ half-cycle, while $74 \%$ were removed during the HF-pyridine half-cycle using the 3-20-2-15 cycle timing. For the 3-60-2-20 cycle timing, $67 \%$ and $33 \%$ were removed in the $\mathrm{NaO}^{t} \mathrm{Bu}$ and $\mathrm{HF}$ pyridine half-cycles, respectively. We note that the polymeric nature of the $\mathrm{NaO}^{t} \mathrm{Bu}$ compound $^{30}$ may lead to mass changes associated with the adsorption and desorption of intact $\mathrm{NaO}^{t} \mathrm{Bu}$ molecules and clusters that do not contribute to the ALD chemistry in Eqs. 1-2 and complicate interpretation of the QCM data. 
$\mathrm{NaF}$ films for ex situ characterization were deposited on $\mathrm{Si}(100)$ coupons via 100 ALD cycles of $\mathrm{NaO}^{t} \mathrm{Bu}+\mathrm{HF}-$ pyridine with 3-20-2-15 cycle timing. X-ray photoelectron spectroscopy (XPS) revealed these films to be nearly stoichiometric NaF. Films grown at $175^{\circ} \mathrm{C}$ and $200{ }^{\circ} \mathrm{C}$ yielded Na:F $=1: 1.05$, while those grown at $225^{\circ} \mathrm{C}$ and $250{ }^{\circ} \mathrm{C}$ yielded atomic ratios of 1:1.02 and 1:0.97, respectively. Figure 3.3 shows survey spectra and narrow scans of the $\mathrm{Na} 1 \mathrm{~s}$ and $\mathrm{F} 1 \mathrm{~s}$ regions for $\mathrm{NaF}$ deposited at $200{ }^{\circ} \mathrm{C}$ with $3-20$ 2-15 cycle timing. Additional XPS spectra for $\mathrm{NaF}$ films deposited at $175^{\circ} \mathrm{C}, 225^{\circ} \mathrm{C}$, and $250{ }^{\circ} \mathrm{C}$ are provided in Appendix B.2. The survey spectra showed incidental contamination with $\mathrm{Ti}$ (all samples) and $\mathrm{Cl}\left(225^{\circ} \mathrm{C}\right.$ and $250^{\circ} \mathrm{C}$ samples), which were attributed to precursor interactions with previously deposited Ti- and Cl-containing materials on the reactor walls. The Si 2 s and Si $2 p$ peaks in the survey scans indicated XPS signal from the substrate were included in the spectra; consequently, the carbon and oxygen peaks likely included signal from the native oxide and pre-existing adventitious carbon layers on the $\mathrm{Si}(100)$ substrates. The relative intensities of the Si peaks were markedly higher for films deposited at $250{ }^{\circ} \mathrm{C}$ (Fig. B7a, inset), which we attribute to film porosity (Figs. 3.6d, B16d, B17d). Although some of the $\mathrm{C}$ and $\mathrm{O}$ signal may have been the result of incomplete ALD surface reactions or decomposition of tert-butoxide ligands, precise chemical state identification was confounded by substrate effects. However, the combined $\mathrm{C}$ and $\mathrm{O}$ content including substrate signal was low (equivalent homogeneous composition of $<4$ at. $\% \mathrm{C}$ and $<2.5$ at.\% $\mathrm{O}$ for all samples), and total carbon and oxygen contamination within the deposited $\mathrm{NaF}$ films is expected to be minimal. 

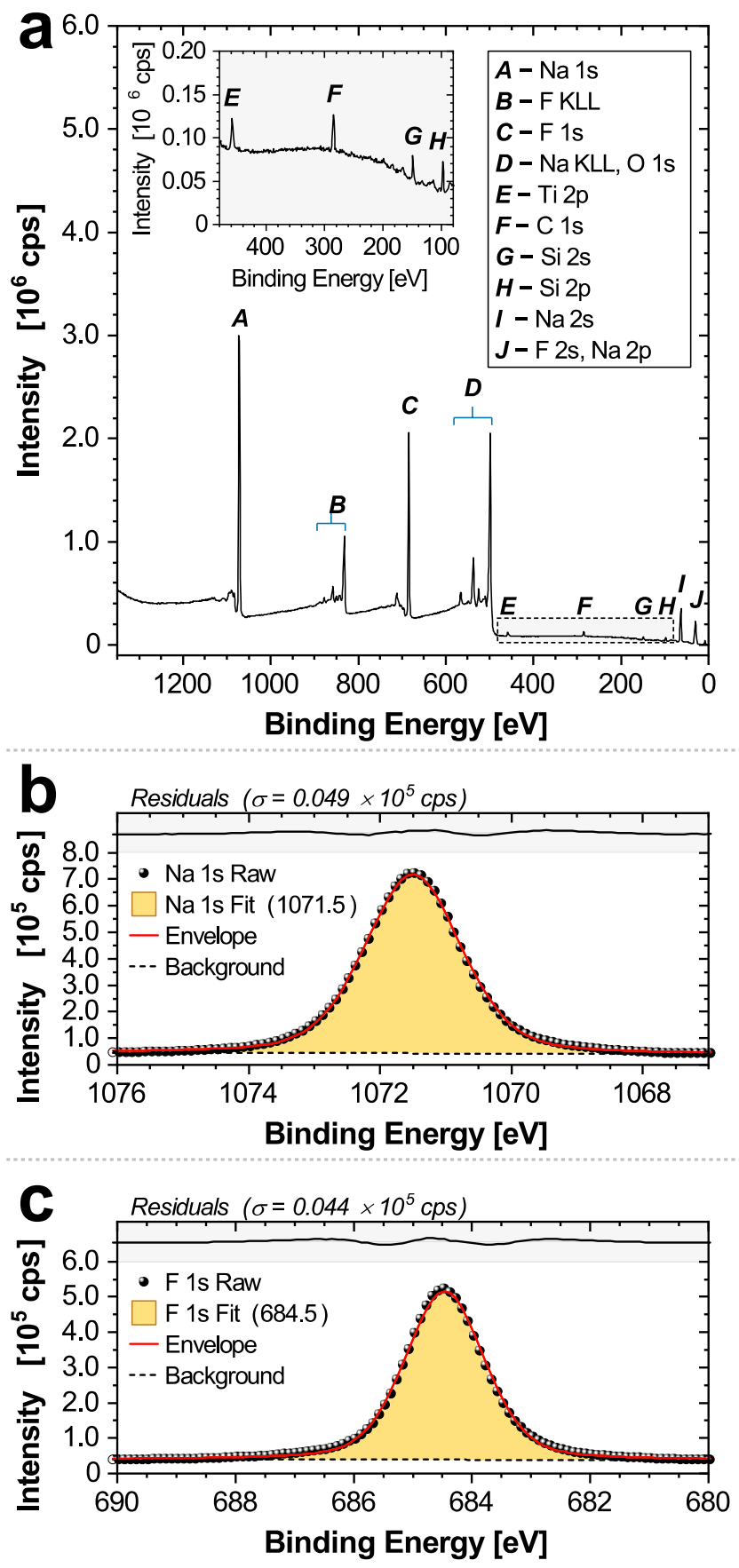

Figure 3.3 XPS data and analysis results for NaF film on silicon substrates. Deposition was performed at $200{ }^{\circ} \mathrm{C}$ with 100 ALD cycles of $\mathrm{NaO}^{t} \mathrm{Bu}+\mathrm{HF}$-pyridine using 3-20-2-15 cycle timing. (a) Survey scan, (b) Sodium 1s region, (c) Fluorine 1s region. 
$\mathrm{NaF}$ films for SE modelling were deposited on Si via 100 ALD cycles of $\mathrm{NaO}^{t} \mathrm{Bu}$ + HF-pyridine with 3-20-2-15 cycle timing. Details on SE modelling and SE data for additional cycle timings are provided in the Appendix B.3. Total NaF film thickness for 100 ALD cycles of $\mathrm{NaO}^{t} \mathrm{Bu}+\mathrm{HF}$-pyridine was $\sim 85 \AA$ at $175^{\circ} \mathrm{C}$ and $200{ }^{\circ} \mathrm{C}$ and increased to $\sim 90 \AA$ and $\sim 111 \AA$ at $225^{\circ} \mathrm{C}$ and $250{ }^{\circ} \mathrm{C}$, respectively. Average NaF growth per cycle (GPC) for various growth temperatures is shown in Figure 3.4. Although all SE fits yielded mean squared error values less than 2, the NaF Sellmeier model deviated from measured Delta data at higher wavelengths for $250{ }^{\circ} \mathrm{C}$ samples (see Appendix B.3 Fig. B13c-d). This discrepancy may be due to film non-uniformity (Fig. 3.6d, Appendix B.4 Fig. B16d, and Appendix B.5 Fig. B17d) and/or incidental chlorine contamination (Appendix B.2 Fig. B7a) at higher growth temperatures. It should be noted that the GPC obtained from SE $\left(\sim 0.85 \AA\right.$ at $\left.200{ }^{\circ} \mathrm{C}\right)$ is less than half of the minimum expected value based on QCM measurements (1.7 $\AA$ ). This discrepancy might be attributed to differences in initial surface chemistry or crystallinity of the ALD NaF films, as the QCM crystal was coated with $\mathrm{Al}_{2} \mathrm{O}_{3}$ prior to each $\mathrm{NaF}$ deposition, while the silicon substrates with native oxide used for SE were directly coated with NaF. To explore this further, SE data were acquired for $\mathrm{NaF}$ deposited on $\mathrm{Al}_{2} \mathrm{O}_{3}$ coated $\mathrm{Si}$ at $200{ }^{\circ} \mathrm{C}$ with $100 \mathrm{ALD}$ cycles of $\mathrm{NaO}^{t} \mathrm{Bu}+\mathrm{HF}-$ pyridine with 3-20-2-15 cycle timing. The data, shown in Figure B15, confirm a higher GPC $\left(\sim 3.2 \AA\right.$ at $\left.200^{\circ} \mathrm{C}\right)$ for growth on $\mathrm{Al}_{2} \mathrm{O}_{3}$, and additional work is needed to understand the differences in nucleation and growth between $\mathrm{Al}_{2} \mathrm{O}_{3}$ and $\mathrm{SiO}_{2}$. 


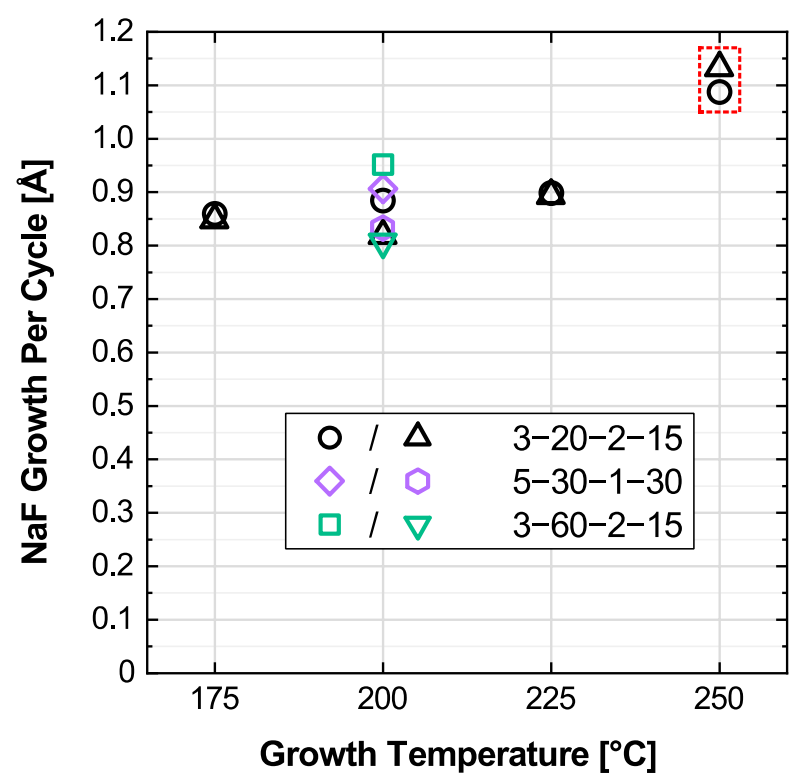

Figure 3.4 Temperature dependence of NaF GPC, based on SE fitting results for 100 ALD cycles of $\mathrm{NaO}^{t} \mathrm{Bu}+\mathrm{HF}$-pyridine on silicon substrates. Duplicate samples from different regions of the reactor chamber (separated by $\sim 50 \mathrm{~cm}$ ) are shown for each growth temperature. Calculated errors of the fitted thickness values are within the data markers. Films deposited at $250{ }^{\circ} \mathrm{C}$ (red) deviated slightly from the bulk NaF Sellmeier SE model, as shown in Appendix B.3 Fig. B13c-d.

To characterize the structures of the deposited films, ex situ grazing incidence Xray diffraction (GIXRD) measurements were performed for $\mathrm{NaF}$ films deposited on $\mathrm{Si}(100)$ coupons via $100 \mathrm{ALD}$ cycles of $\mathrm{NaO}^{t} \mathrm{Bu}+\mathrm{HF}-$ pyridine with 3-20-2-15 cycle timing. The GIXRD data are shown in Figure 3.5 for samples grown at 175, 200, 225 , and $250^{\circ} \mathrm{C}$. Red ticks indicate the reflections for cubic NaF, which were computed using VESTA $^{33}$ using structure data for NaF (ICSD 53840). ${ }^{34,35}$ For all deposition temperatures, the data indicate good agreement with the cubic NaF structure. 


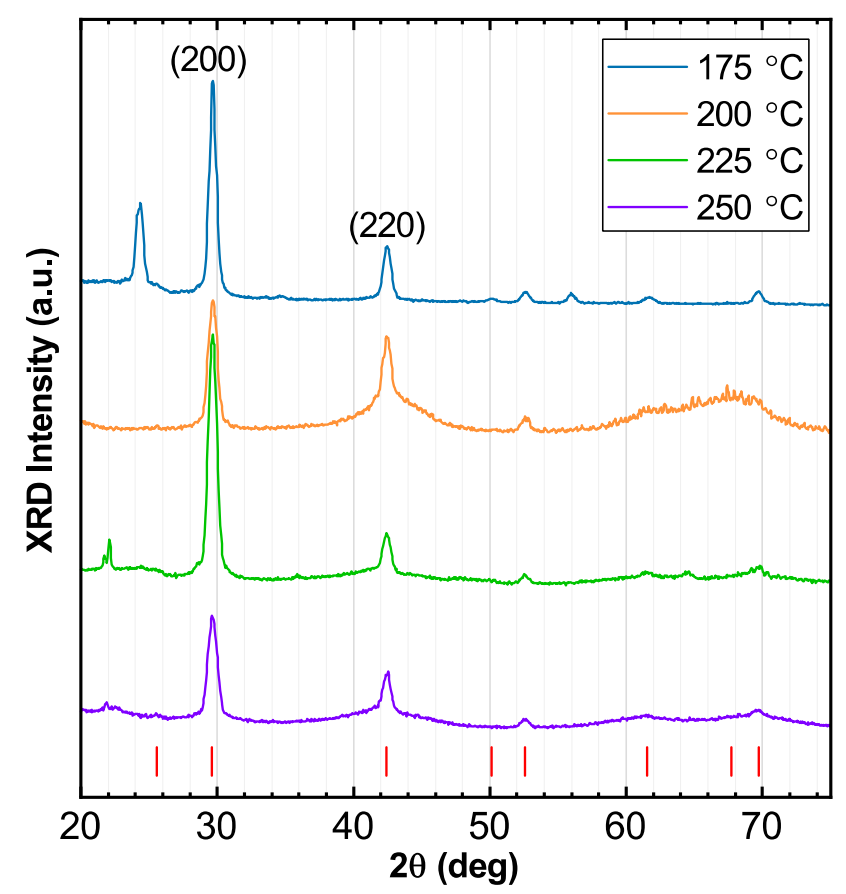

Figure 3.5 Grazing incidence X-ray diffraction (GIXRD) data for NaF films deposited on silicon substrates with $100 \mathrm{ALD}$ cycles of $\mathrm{NaO}^{t} \mathrm{Bu}+\mathrm{HF}$-pyridine. Data are shown for films grown at $175,200,225$, and $250^{\circ} \mathrm{C}$, and red ticks indicate the predicted reflections for cubic $\mathrm{NaF}$.

Finally, atomic force microscopy and scanning electron microscopy were used to characterize the film morphology. AFM images for as-deposited samples are shown in Figure 3.6 (see Appendices B.4 and B.5 for AFM and SEM images of air-exposed samples). Root mean square roughness $\left(\mathrm{R}_{\mathrm{q}}\right)$ values for the $175^{\circ} \mathrm{C}$ and $200{ }^{\circ} \mathrm{C}$ asdeposited samples were both $\sim 1.6 \mathrm{~nm}$ for films of roughly $8.5 \mathrm{~nm}$ as determined from SE of similar samples. Roughness values increased with temperature; the measured $\mathrm{R}_{\mathrm{q}}$ values were $\sim 2.2 \mathrm{~nm}$ at $225^{\circ} \mathrm{C}$ for a film of $\sim 9.0 \mathrm{~nm}$ thickness and $\sim 5.8 \mathrm{~nm}$ at $250{ }^{\circ} \mathrm{C}$ for a film of $11.1 \mathrm{~nm}$. We note that film thicknesses were measured in air using SE for samples equivalent to those measured via $\mathrm{AFM}$, and that $\mathrm{R}_{\mathrm{q}}$ values of several $\mathrm{nm}$ are typical for polycrystalline ALD films in this thickness range, whereas amorphous ALD films in this thickness range exhibit $R_{q}$ values well below $1 \mathrm{~nm} .{ }^{36}$ This finding is sensible given that $\mathrm{NaF}$ is an ionic solid and should readily crystallize even at low growth temperatures, as 
confirmed by GIXRD (Fig. 3.5). Air-exposed samples showed similar trends in roughness at elevated growth temperatures and had $\mathrm{R}_{\mathrm{q}}$ values slightly higher than asdeposited samples (Fig. 3.6e). This increase in $\mathrm{R}_{\mathrm{q}}$ for air-exposed films may be due to water adsorption. $\mathrm{NaF}$ is known to be hygroscopic and the adsorption of $\mathrm{H}_{2} \mathrm{O}$ vapor from the ambient environment would cause volume expansion and an increase in surface roughness. Film morphology evolved with temperature, with small pores and cubic crystallite structures forming on the $\mathrm{NaF}$ surface at $250^{\circ} \mathrm{C}$, as shown in Fig. $3.6 \mathrm{~d}$ (also Appendix B.4 Fig. B16d, Appendix B.5 Fig B17d). The pores may have resulted from de-wetting of the ALD NaF from the native silicon oxide surface at the higher growth temperatures. 

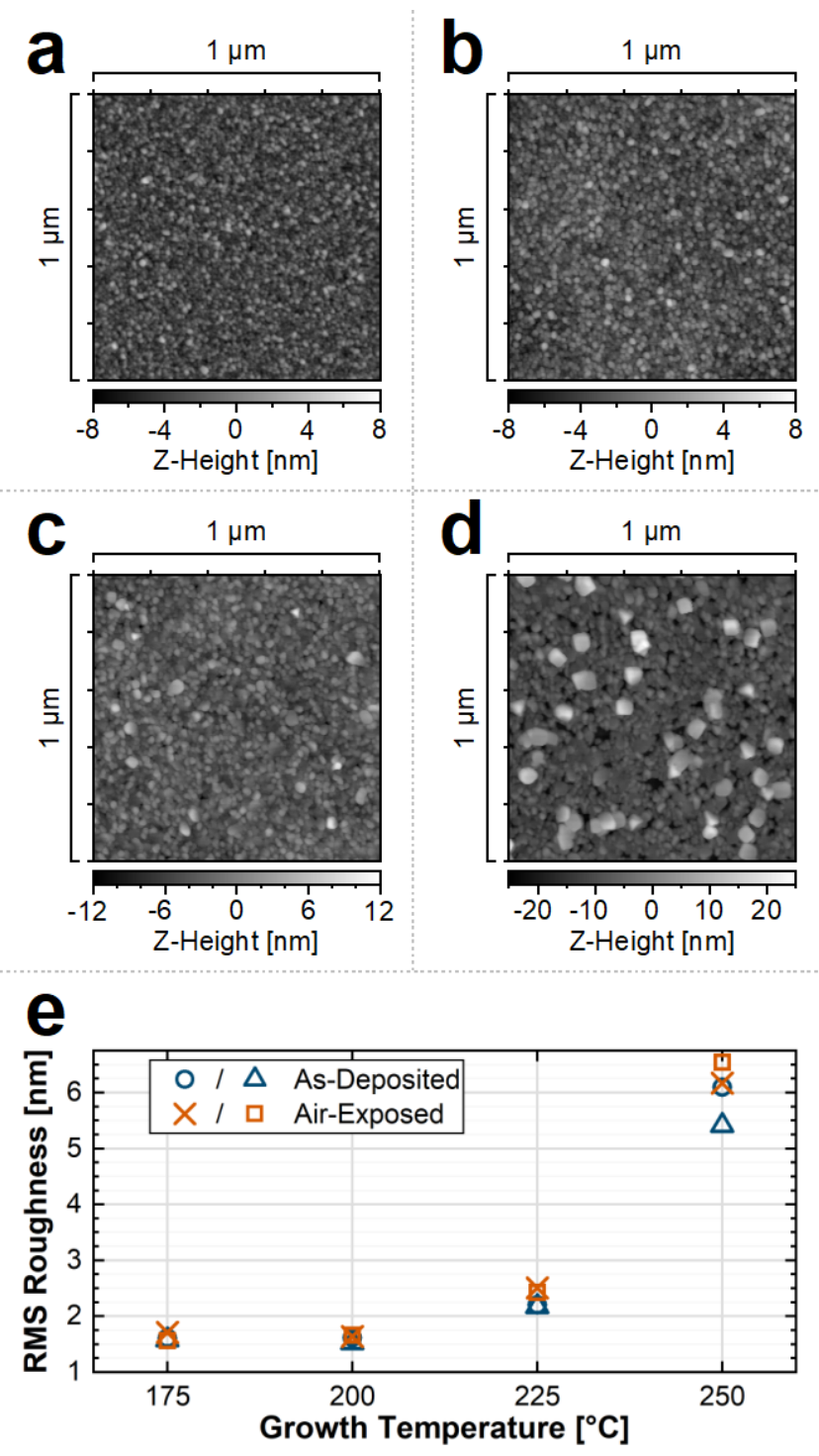

Figure 3.6 Argon-filled glovebox AFM images of NaF surface morphology for asdeposited films on silicon substrates at growth temperatures of (a) $175^{\circ} \mathrm{C}$, (b) 200 ${ }^{\circ} \mathrm{C}$, (c) $225^{\circ} \mathrm{C}$, and (d) $250{ }^{\circ} \mathrm{C}$. (e) Surface roughness $\mathrm{R}_{\mathrm{q}}$ values for various growth temperatures (blue $=$ as-deposited, orange $=$ air-exposed). See Appendix B.4 for AFM images of air-exposed samples.

\subsection{Summary and Conclusions}

We reported a binary chemistry for ALD of NaF with an optimal growth temperature of $175-200^{\circ} \mathrm{C}$. Films deposited in this temperature range were polycrystalline with a cubic crystal structure, had an atomic ratio of $\mathrm{Na}: \mathrm{F}=1: 1.05$, and surface roughness of $1.6 \mathrm{~nm}$ for $8.5 \mathrm{~nm}$ thick films on Si. Optical properties were 
consistent with bulk values and growth per cycle was roughly $0.85 \AA$ on $\mathrm{Si}$ and up to 3.2 $\AA$ on $\mathrm{Al}_{2} \mathrm{O}_{3}$. Films grown on $\mathrm{Si}$ at higher temperatures of $225-250{ }^{\circ} \mathrm{C}$ had increased surface roughness $(2.2$ and $5.8 \mathrm{~nm}$, respectively), with pores and cubic surface crystallites forming at $250{ }^{\circ} \mathrm{C}$. Standard timing for the $\mathrm{NaO}^{t} \mathrm{Bu}+\mathrm{HF}$-pyridine ALD cycle was 3-20$2-15 \mathrm{~s}$. Increased purge time following the $\mathrm{NaO}^{t} \mathrm{Bu}$ dose led to the decomposition and loss of $\mathrm{O}^{t} \mathrm{Bu}$ ligands from the growth surface, but binary $\mathrm{NaF}$ ALD was largely unaffected by the instability of $\mathrm{O}^{t} \mathrm{Bu}$ groups as subsequent HF-pyridine doses re-saturated the growth surface. These results may offer significant benefit for applications of $\mathrm{NaF}$ thin films in applications for both solar cells and advanced batteries.

\subsection{Acknowledgments}

The work of A.M., D.K., and J.E. was supported as part of the Center for Electrochemical Energy Science, an Energy Frontier Research Center funded by the U.S. Department of Energy, Office of Science, Office of Basic Energy Sciences. The work of S.K., J.S., and E.G. at Boise State University was supported by Department of Energy Award no. DE-SC0019121. Atomic force microscope images were obtained in the Surface Science Laboratory at Boise State University (special thanks to Sophia Mitchell and Audrey Parker for collecting glovebox AFM images). Scanning electron microscopy images were obtained by Dr. Nick Bulloss at the Boise State Center for Materials Characterization. This research used the beamline sector 33-ID-D of the Advanced Photon Source, a U.S. Department of Energy (DOE) Office of Science User Facility, operated for the DOE Office of Science by Argonne National Laboratory under Contract No. DE-AC02-06CH11357. Extraordinary facility operations were supported in part by the DOE Office of Science through the National Virtual Biotechnology Laboratory, a 
consortium of DOE national laboratories focused on the response to COVID-19, with funding provided by the Coronavirus CARES Act. S.K. and E.G. acknowledge Dr. Steven Hues and Dr. Kent Zhuang for valuable discussions of XPS.

\subsection{Data Availability}

The data that support the findings of this study are available within the article and its supplemental material (Appendix B).

\subsection{References}

${ }^{1}$ F. Wu, J. Maier and Y. Yu, Chemical Society Reviews 49 (5), 1569-1614 (2020).

${ }^{2}$ M. Walter, M. V. Kovalenko and K. V. Kravchyk, New Journal of Chemistry 44 (5), $1677-$ 1683 (2020).

${ }^{3}$ W. Luo, F. Shen, C. Bommier, H. Zhu, X. Ji and L. Hu, Accounts of Chemical Research 49 (2), 231-240 (2016).

${ }^{4}$ X. Pu, H. Wang, D. Zhao, H. Yang, X. Ai, S. Cao, Z. Chen and Y. Cao, Small 15 (32), 1805427 (2019).

${ }^{5}$ J. Biemolt, P. Jungbacker, T. van Teijlingen, N. Yan and G. Rothenberg, Materials 13 (2) 425 (2020).

${ }^{6}$ F. Yu, L. Du, G. Zhang, F. Su, W. Wang and S. Sun, Advanced Functional Materials 30 (9), 1906890 (2020).

${ }^{7}$ D. Zuo, G. Tian, X. Li, D. Chen and K. Shu, Journal of Alloys and Compounds 706, 24-40 (2017).

${ }^{8}$ B. Ahmed, C. Xia and H. N. Alshareef, Nano Today 11 (2), 250-271 (2016).

${ }^{9}$ Y. Zhao, K. Zheng and X. Sun, Joule 2 (12), 2583-2604 (2018).

${ }^{10}$ J. Liu, H. Zhu and M. H. A. Shiraz, Frontiers in Energy Research 6 (10) (2018).

${ }^{11}$ L. Ma, R. B. Nuwayhid, T. Wu, Y. Lei, K. Amine and J. Lu, Advanced Materials Interfaces 3 (21), 1600564 (2016). 
${ }^{12}$ T. Kääriäinen, D. Cameron, M. L. Kääriäinen and A. Sherman, Atomic Layer Deposition: Principles, Characteristics, and Nanotechnology Applications, 2 ed. (Scrivener Publishing, Beverly, MA, 2013).

${ }^{13}$ S. M. George, Chemical Reviews 110 (1), 111-131 (2010).

${ }^{14}$ M. Mäntymäki, M. Ritala and M. Leskelä, Coatings 8 (8) 277 (2018).

${ }^{15}$ M. Feinauer, H. Euchner, M. Fichtner and M. A. Reddy, ACS Applied Energy Materials 2 (10), 7196-7203 (2019).

${ }^{16}$ Y. Lee, H. Sun, M. J. Young and S. M. George, Chemistry of Materials 28 (7), 2022-2032 (2016).

${ }^{17}$ L. Chen, K.-S. Chen, X. Chen, G. Ramirez, Z. Huang, N. R. Geise, H.-G. Steinrück, B. L. Fisher, R. Shahbazian-Yassar, M. F. Toney, M. C. Hersam and J. W. Elam, ACS Applied Materials \& Interfaces 10 (32), 26972-26981 (2018).

${ }^{18}$ I. Hwang, S.-K. Jung, E.-S. Jeong, H. Kim, S.-P. Cho, K. Ku, H. Kim, W.-S. Yoon and K. Kang, Nano Research 10 (12), 4388-4397 (2017).

${ }^{19}$ Z. W. Seh, J. Sun, Y. Sun and Y. Cui, ACS Cent Sci 1 (8), 449-455 (2015).

${ }^{20}$ R. Binions, C. J. Carmalt and I. P. Parkin, Thin Solid Films 469-470, 416-419 (2004).

${ }^{21}$ L. J. Lingg, A. D. Berry, A. P. Purdy and K. J. Ewing, Thin Solid Films 209 (1), 9-16 (1992).

${ }^{22}$ J. A. Samuels, W.-C. Chiang, C.-P. Yu, E. Apen, D. C. Smith, D. V. Baxter and K. G. Caulton, Chemistry of Materials 6 (10), 1684-1692 (1994).

${ }^{23}$ C. Andres, T. Schwarz, S. G. Haass, T. P. Weiss, R. Carron, R. Caballero, R. Figi, C. Schreiner, M. Bürki, A. N. Tiwari and Y. E. Romanyuk, Solar Energy 175, 94-100 (2018).

${ }^{24}$ N. Li, S. Tao, Y. Chen, X. Niu, C. K. Onwudinanti, C. Hu, Z. Qiu, Z. Xu, G. Zheng, L. Wang, Y. Zhang, L. Li, H. Liu, Y. Lun, J. Hong, X. Wang, Y. Liu, H. Xie, Y. Gao, Y. Bai, S. Yang, G. Brocks, Q. Chen and H. Zhou, Nature Energy 4 (5), 408-415 (2019). 
${ }^{25}$ G. Rajan, B. Belfore, S. Karki, D. Poudel, H. Kahoui, N. Lanham, E. Palmiotti, S. Soltanmohammad, A. Rockett and S. Marsillac, Thin Solid Films 690, 137526 (2019).

${ }^{26}$ D. Ledinek, J. Keller, C. Hägglund, W.-C. Chen and M. Edoff, Thin Solid Films 683, 156164 (2019).

${ }^{27}$ H. H. Sønsteby, O. Nilsen and H. Fjellvåg, Journal of Vacuum Science \& Technology A 34 (4), 041508 (2016).

${ }^{28}$ H. H. Sønsteby, O. Nilsen and H. Fjellvåg, Global Challenges 3 (10), 1800114 (2019).

${ }^{29}$ E. Østreng, H. H. Sønsteby, S. Øien, O. Nilsen and H. Fjellvåg, Dalton Transactions 43 (44), 16666-16672 (2014).

${ }^{30}$ H. H. Sønsteby, J. E. Bratvold, V. A. L. K. Killi, D. Choudhury, J. W. Elam, H. Fjellvåg and O. Nilsen, Journal of Vacuum Science \& Technology A 38 (6), 060804 (2020).

${ }^{31}$ J. W. Elam, M. D. Groner and S. M. George, Review of Scientific Instruments 73 (8), 29812987 (2002).

${ }^{32}$ D. Nečas and P. Klapetek, Open Physics 10 (1), 181-188 (2012).

${ }^{33}$ K. Momma and F. Izumi, Journal of Applied Crystallography 44 (6), 1272-1276 (2011).

${ }^{34}$ G. Bergerhoff, I. Brown and F. Allen, International Union of Crystallography, Chester 360, 77-95 (1987).

${ }^{35}$ P. Debye and P. Scherrer, Physikalische Zeitschrift 19, 474-483 (1918).

${ }^{36}$ J. W. Elam, Z. A. Sechrist and S. M. George, Thin Solid Films 414 (1), $43-55$ (2002). 


\section{CHAPTER FOUR: CONCLUSIONS AND FUTURE DIRECTIONS}

We have presented results for GaP films grown on a variety of substrates using $\mathrm{TMGa}+\mathrm{TDMAP}$ and TMGa + TBP. Both chemistries required static dosing to increase precursor exposure and achieve film growth without prohibitively long precursor doses, and showed inconsistencies thought to be the result of pre-deposition contamination on the substrate surface. Future investigations of GaP ALD using these chemistries would be greatly improved by the development of a high-temperature QCM that does not require that the back side of the crystal be purged with inert gas. A purgeless QCM of this type would allow for investigation of GaP ALD via static dosing, which our results indicate is necessary to achieve GaP growth with reasonable precursor dose times.

In an effort to better understand GaP ALD, extensive modifications were made to a custom tube-furnace ALD reactor, and a new LabVIEW control program was developed to incorporate execution of ALD experiments with measurement of process parameters, as well as real-time data analysis to aid in ALD process development. This LabVIEW VI allows for automation of complex ALD recipes with pre-programmed changes in numerous process variables, including ALD precursor dosing parameters, carrier gas flow rates, and reactor temperature ramps. Each recipe step can also be set to simultaneously open a valve for an attached mass spectrometer, enabling efficient fullscale time-resolved mass spectroscopy to investigate ALD reaction pathways, as was recently reported with an alternative pathway for ALD of trimethylaluminum and water. ${ }^{1}$ Planned future modifications to the LabVIEW control program include full 
implementation of automated FTIR scans into the ALD recipe block-diagram execution code. Furthermore, work will be done to automate data post-processing, including timeresolution analysis for mass spectrometry measurements and exports of formatted plot images for all recorded data. In particular, the ability to correlate purgeless QCM measurements with mass and FTIR spectroscopy would provide significantly greater insight into the surface reactions involved in the GaP ALD processes, as well as any other ALD process. When combined with ex situ characterization, these comprehensive data sets may provide a path for reliable ALD of GaP, including possible area-selective deposition suggested by preliminary results on $\mathrm{H}-\mathrm{Si}(100)$.

We have also reported a newly-developed process for ALD of $\mathrm{NaF}$ thin films, with an optimal growth temperature of $175-200{ }^{\circ} \mathrm{C}$ and resulting films consistent with nearly stoichiometric NaF. Future work utilizing ALD of NaF may include additional process characterization to resolve the discrepancy between the ALD growth rates on $\mathrm{Si}(100)$ with native oxide (as measured via SE) and ALD alumina (as calculated from QCM results). Electrochemical measurements of NaF anode coatings in button-cell batteries are also planned.

\section{References}

1. Werbrouck, A.; Shirazi, M.; Mattelaer, F.; Elliott, S. D.; Dendooven, J.; Detavernier, C., A Secondary Reaction Pathway for the Alumina Atomic Layer Deposition Process with Trimethylaluminum and Water, Revealed by Full-Range, TimeResolved In Situ Mass Spectrometry. The Journal of Physical Chemistry C 2020, 124 (48), 26443-26454. 
APPENDIX A

Additional Details on Labview ALD Reactor Control Program 


\section{A.1 Initialization: Cluster Arrays}

To improve program modularity and simplify precursor capacity expansion, a cluster array global variable was used to store all precursor configuration details. Figure A1 illustrates one element of the cluster array for precursor A1 (TMA). The array element contains details on the precursor's label and name, typical needle valve settings used, its enabled/disabled setting, and Boolean clusters specifying the precursor's digital output (DO) configurations for each of the system's cDAQ DO modules. Precursors are added by initializing their settings within this cluster array, which is then used by other VIs during program operation. Default values stored in the cluster array are automatically populated in the main VI's front-panel controls, as shown in Figure 2.4. Precursors that are configured but set to Disabled in this cluster array have their corresponding frontpanel controls disabled and greyed out at run-time, and they are not available for ALD recipe execution. Similar cluster arrays (not shown) were used for initialization of pressure gauges, mass flow controllers, and thermocouples. All array elements initialized and enabled in the set-up files are automatically added to DAQmx read/write tasks during program operation. 


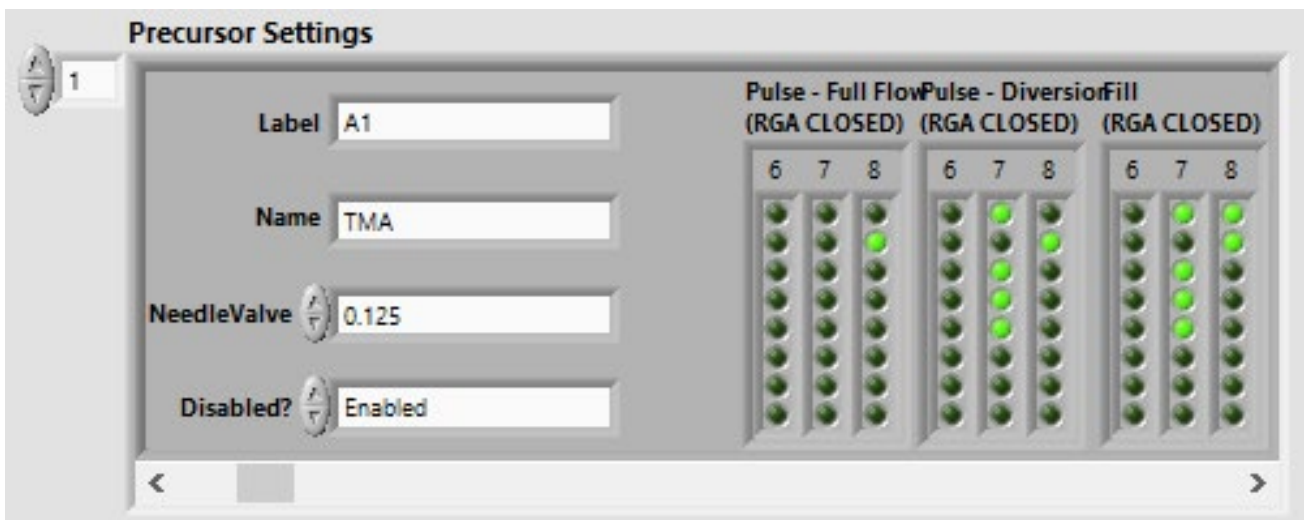

\section{Figure A1 Screenshot of Precursor Settings cluster array global variable used for initialization of chemical precursors. Settings configured in this array are utilized throughout the rest of the LabVIEW program.}

\section{A.2 Data Collection and Processing}

After initialization, the program uses five while-loops running in parallel within the main VI. Operations for data collection (Loop 1), data processing (Loop 2), graph and system indicator updates (Loop 3), response to miscellaneous user controls (Loop 4), and manual reactor control and ALD recipe execution (Loop 5) are handled separately to allow each operation's execution rate to be configured based on its priority level. Figure A2 shows Loops 1 and 2, while Figure A3 shows Loops 3-5.

Loops 1 and 2 utilize a producer/consumer architecture to minimize the impact of time-consuming data processing and file writing tasks on the rate of data collection. In this approach, a producer loop executes continuously, collecting raw data measurements and corresponding time stamps at specified time intervals (in this case, $500 \mathrm{~ms}$ was used). Within the CollectData subVI, analog voltage measurements from the ALD reactor components (MFCs, TCs, and pressure gauges) are collected using DAQmx device drivers, and QCM measurements are collected via the STM-x_HAL LabVIEW library for serial communication with STM-2 Film Thickness Monitors, which is available directly from Inficon. Measured data are placed directly into a queue within the producer loop 
and are then de-queued in the consumer loop, where hardware-specific scaling factors are applied to convert voltage measurements into meaningful flow and pressure values within the ProcessData subVI. If the VI is placed in Simulation Mode before it is run, data is instead generated in a simulation subVI (not shown) and placed in the queue for processing. By using queues to pass data between separate loops in this manner, data collection proceeds at the desired rate without being bogged down by the slower processing and file writing tasks. After completing all calculations on the de-queued data, the ProcessData subVI passes the data to an action engine VI, which stores data arrays used to update the front-panel graphs. If an ALD recipe is executing with data logging enabled, a file writing VI periodically retrieves processed data from global Data Log arrays, then formats the numerical data into text strings and appends them to appropriate $\log$ files. 


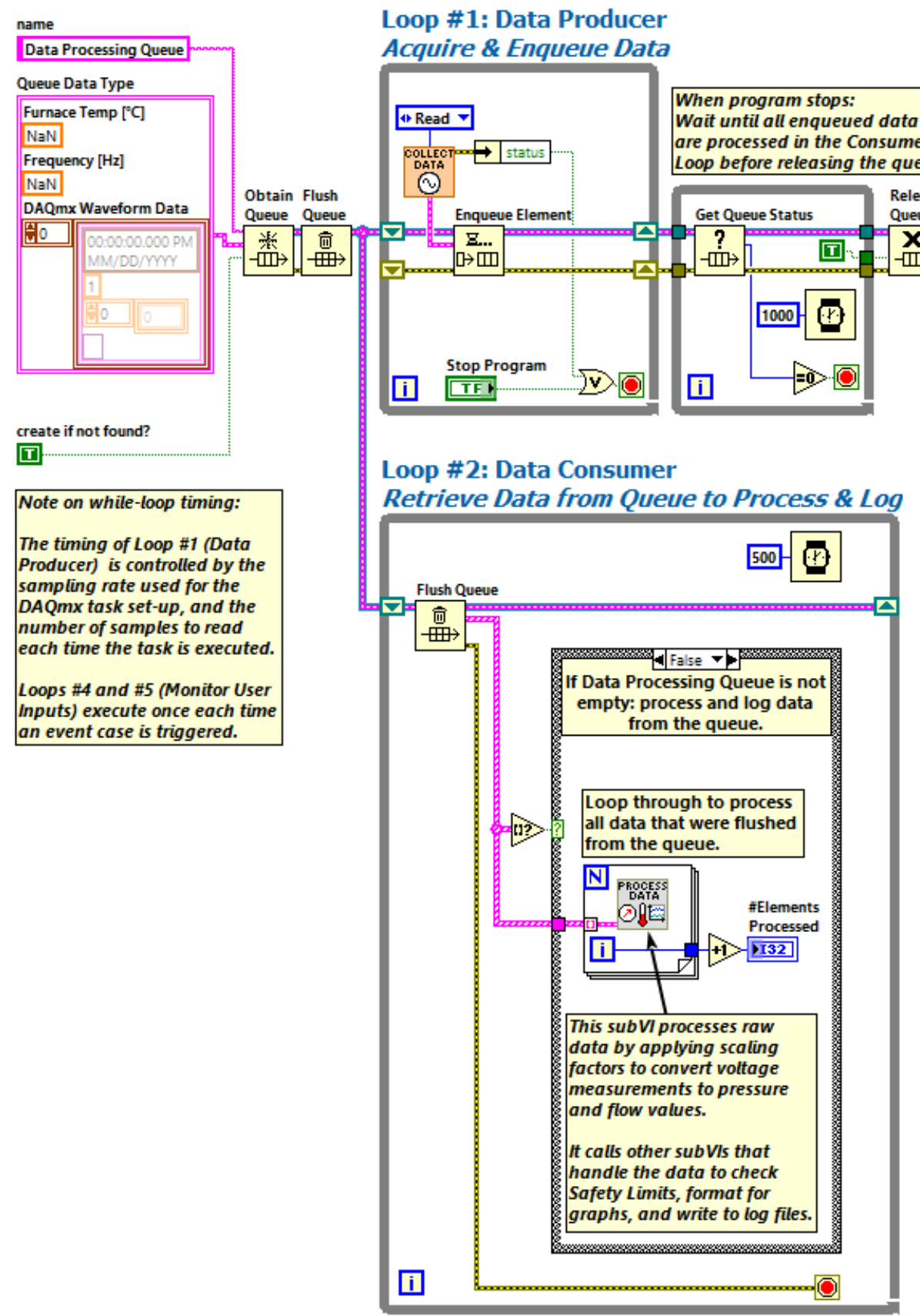

Figure A2 Screenshot of VI block diagram section showing Producer/Consumer programming architecture used to continuously collect, process, and log ALD reactor data. 


\section{A.3 Reactor Operation and ALD Recipe Execution}

Loop 5 contains an event case structure used to monitor and respond to all user inputs related to manual system control, sending user commands to the cDAQ modules to operate valves or change MFC setpoints. When the user adds or deletes recipe steps, this loop and event case also modifies the ALD recipe, which is stored in its full form as a cluster array global variable, as shown in Figure A4. Each cluster array element contains information on a single recipe step, including the recipe step type (e.g., purge/wait step, ALD precursor cycle, MFC change, temp change, etc.), selection of RGA mass spectroscopy, and a variant with essential parameters for execution of the step (e.g., dose time and purge time). Finally, the event case structure in Loop 4 handles execution of ALD recipe steps, as shown in Figure A3. Before recipe execution begins, a series of checks is performed to ensure no problems exist with the ALD recipe or with data logging settings. If no errors are found and measured parameters are within the specified Safety Limits, the graphs are cleared and the Start Time is reset, and manual valve controls are returned to their default states. Aside from the Stop Recipe button, all other user controls for manual system operation or recipe modification are disabled while an ALD recipe is in progress. The ALD recipe is then executed in a step-by-step fashion until the recipe is stopped by one of three conditions: 1) the recipe reaches completion after all steps are successfully executed, 2) the recipe is manually terminated by the user, or 3 ) the recipe is automatically terminated when measured parameters fall outside the specified Safety Limits. Figure A5 shows the block diagram of the ExecuteRecipeStep subVI for the case of a Fill-Hold-Purge recipe step. A single cluster element of the Recipe Execution Array is extracted, and the cluster is unbundled to determine the recipe 
step type. The corresponding valve configurations are indexed from global variables Misc. Valves and Precursor Info, recipe parameter variants are converted to the corresponding data types for the recipe step, and then each portion of the step is executed in corresponding recipe step subVIs (in this case, Fill, Hold, and Purge subVIs). If the recipe is manually stopped by the user, or if measured parameters fall outside the specified Safety Limits at any time during execution of the recipe step subVIs, recipe execution will immediately terminate. The reason for termination is noted on the frontpanel Recipe Status indicator and recorded to the appropriate log file if data logging is enabled. 

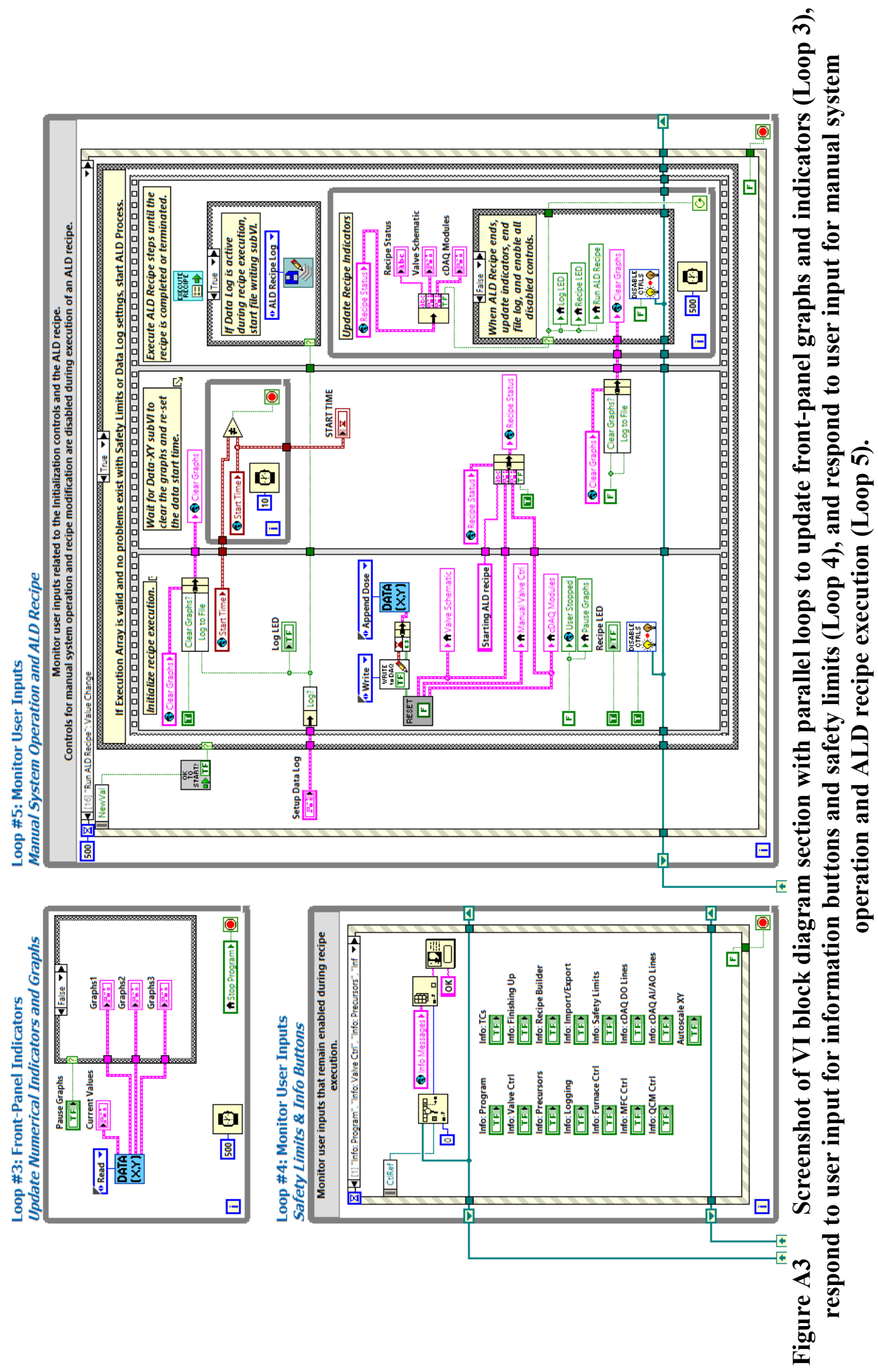


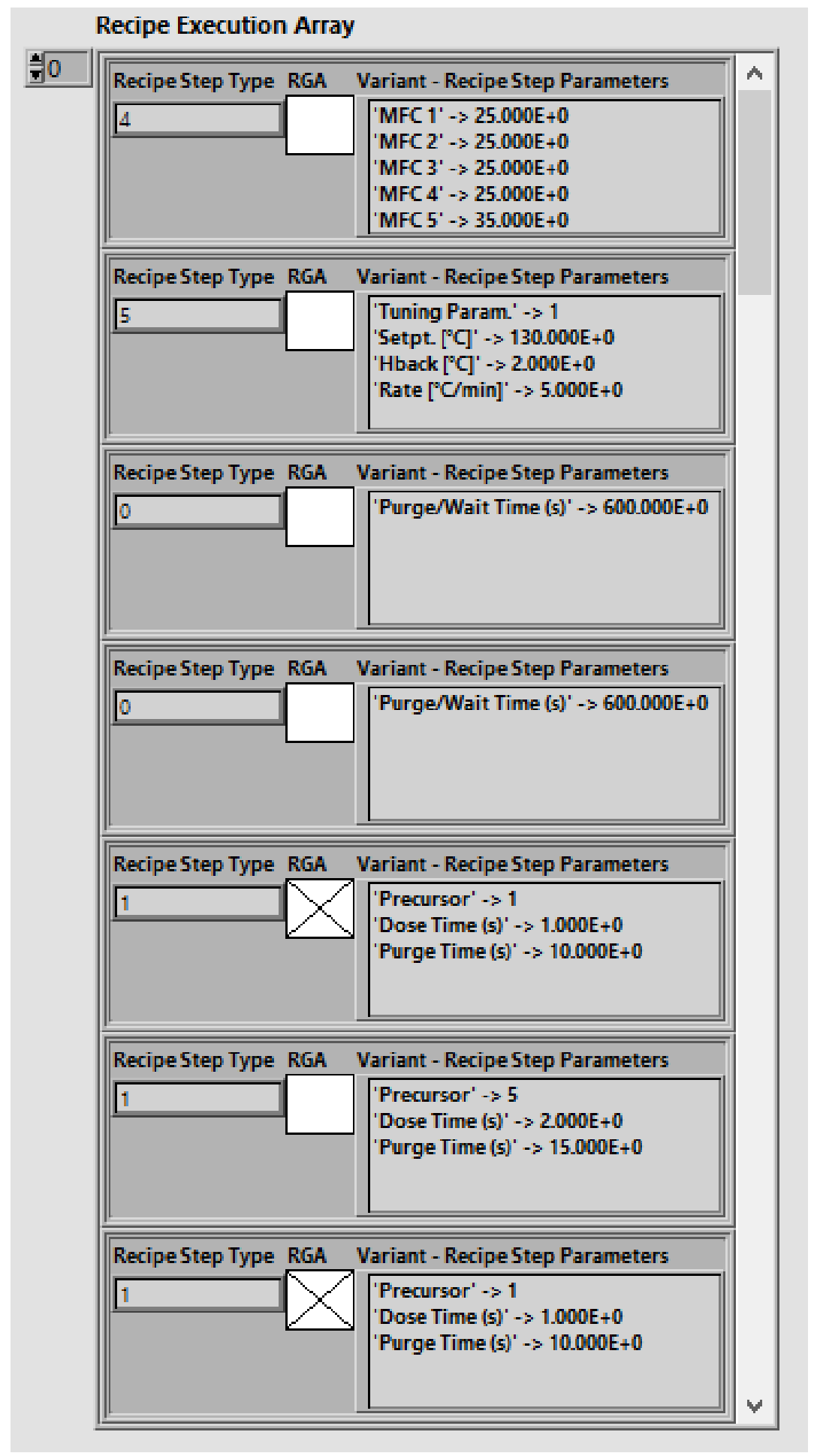

Figure A4 Screenshot of first 7 lines of the Recipe Execution Array corresponding to the ALD Recipe shown in Figure 2.5. The Recipe Execution Array is stored as a global variable and accessed during recipe modification, recipe execution, and file writing. Each cluster array element contains all information needed for execution of a single recipe step. 


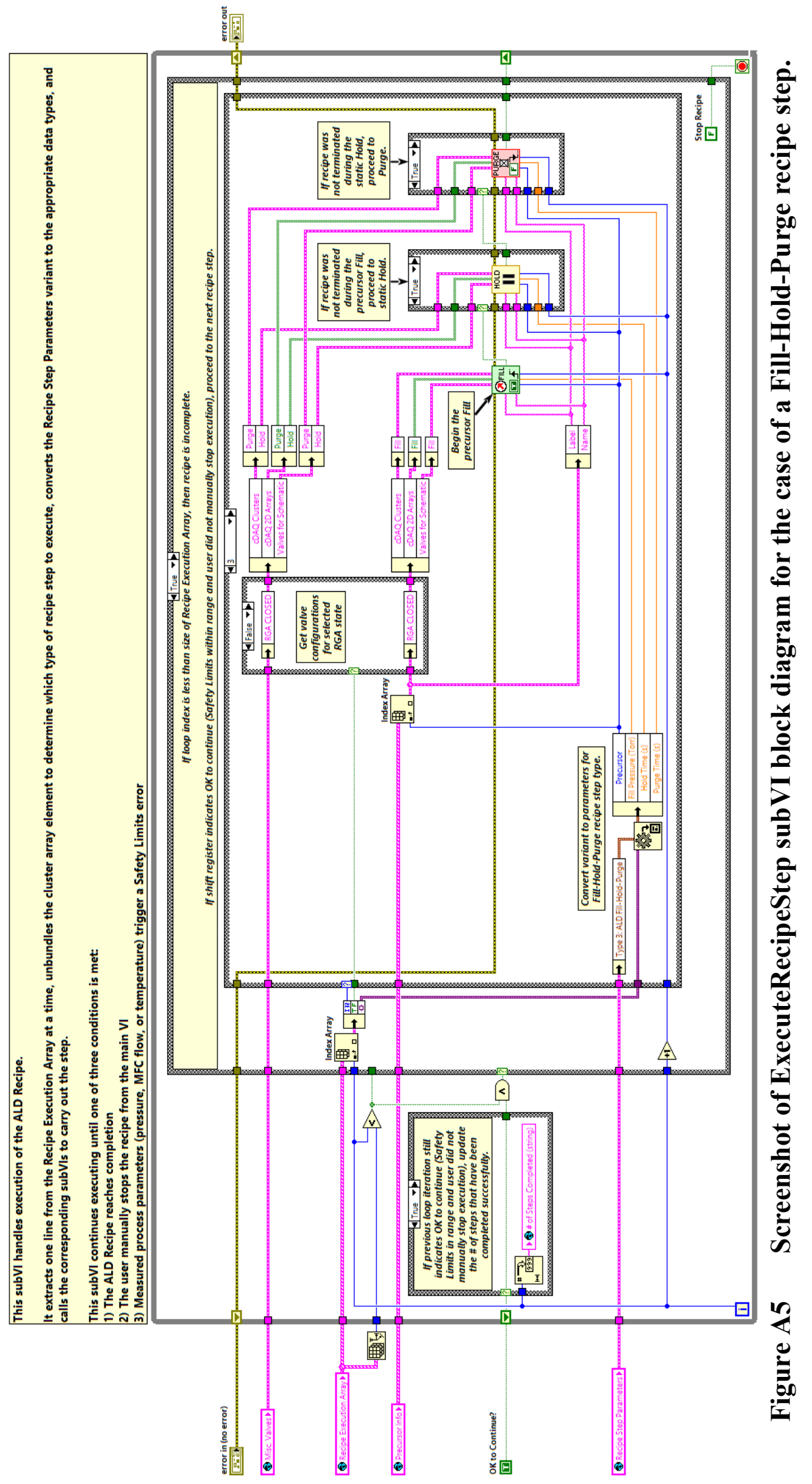




\section{APPENDIX B}

\section{Supplemental Information For NaF ALD}

This material was submitted as supplemental information for publication of "Atomic Layer Deposition of Sodium Fluoride Thin Films" in JVST A. Reproduced from Journal of Vacuum Science \& Technology A 39, 032405 (2021) with permission of AIP publishing. For this dissertation appendix, additional discussion was added regarding the use of the Sellmeier model for spectroscopic ellipsometry.

https://doi.org/10.1116/6.0000847

Sara Kuraitis ${ }^{1}$, Donghyeon Kang ${ }^{2}$, Anil U. Mane ${ }^{2}$, Hua Zhou ${ }^{3}$, Jake Soares ${ }^{1}$, Jeffrey W. Elam ${ }^{2, a)}$, Elton Graugnard ${ }^{1, b)}$

${ }^{1}$ Micron School of Materials Science \& Engineering, Boise State University, 1910 University Dr., Boise, Idaho 83725

${ }^{2}$ Applied Materials Division, Argonne National Laboratory, 9700 S. Cass Ave, Argonne, Illinois 60439

${ }^{3}$ X-ray Science Division, Advanced Photon Source, Argonne National

Laboratory, Lemont, IL 60439

a) Electronic mail: jelam@anl.gov,

b) Electronic mail: eltongraugnard@boisestate.edu 


\section{B.1 QCM for Varied Dosing Parameters}

Prolonged purge time following $\mathrm{NaO}^{t} \mathrm{Bu}$ doses led to a change in process

behavior as observed via QCM. When alternating $\mathrm{NaO}^{t} \mathrm{Bu}$ and $\mathrm{HF}-$ pyridine doses, the

overall shape of the QCM graph changed $\left(\left|\Delta \mathrm{m}_{\mathrm{NaO}}{ }_{\mathrm{Bu}}\right|>>\left|\Delta \mathrm{m}_{\mathrm{HF}}\right|\right)$, but the total mass

change per cycle remained similar across all dose and purge times explored (Table B1,

Figure B1). Average mass change values for each cycle timing variation are shown in

Figure $\mathrm{B} 2$. Repeated $\mathrm{NaO}^{t} \mathrm{Bu}$ doses with 60 second purges were not self-limiting, while those with 10 second purges quickly reached saturation (Figure B3).

Table $\mathrm{B} 1 \quad \mathrm{NaO}^{t} \mathrm{Bu}+\mathrm{HF}$-pyridine dose and purge times for data shown in Figure B1. All time values are in seconds.

\begin{tabular}{|c|c|c|c|c|c|}
\hline Figure B1 & Cycle \# & $\begin{array}{c}\mathrm{NaO}^{t} \mathrm{Bu} \\
\text { Dose (t1) } \\
\end{array}$ & $\begin{array}{c}\mathrm{NaO}^{t} \mathrm{Bu} \\
\text { Purge (t2) } \\
\end{array}$ & $\begin{array}{c}\text { HF-pyr } \\
\text { Dose (t3) }\end{array}$ & $\begin{array}{c}\text { HF-pyr } \\
\text { Purge (t4 }\end{array}$ \\
\hline $\mathbf{a}$ & $1-20$ & 3 & 20 & 2 & 15 \\
\hline b & $1-2$ & 3 & 15 & 2 & 15 \\
\hline b & $3-4$ & 3 & 20 & 2 & 20 \\
\hline b & $5-6$ & 3 & 30 & 2 & 20 \\
\hline b & $7-8$ & 3 & 45 & 2 & 20 \\
\hline b & $9-11$ & 3 & 60 & 2 & 20 \\
\hline b & $12-16$ & 3 & 20 & 2 & 20 \\
\hline b & $17-18$ & 3 & 20 & 1 & 20 \\
\hline c & $1-3$ & 3 & 20 & 1 & 20 \\
\hline c & $4-7$ & 3 & 20 & 4 & 20 \\
\hline c & $8-10$ & 3 & 60 & 4 & 20 \\
\hline d & 1 & 3 & 60 & 2 & 20 \\
\hline d & $2-5$ & 3 & 120 & 2 & 20 \\
\hline d & 6 & 10 & 120 & 2 & 20 \\
\hline d & 7 & 10 & 120 & 2 & 30 \\
\hline d & $8-9$ & 3 & 120 & 5 & 20 \\
\hline
\end{tabular}

\begin{tabular}{|c|c|c|c|c|c|}
\hline Figure B1 & Cycle \# & $\begin{array}{c}\mathbf{N a O}^{t} \mathbf{B u} \\
\text { Dose (t1) }\end{array}$ & $\begin{array}{c}\mathbf{N a O}^{t} \text { Bu } \\
\text { Purge (t2) }\end{array}$ & $\begin{array}{c}\text { HF-pyr } \\
\text { Dose (t3) }\end{array}$ & $\begin{array}{c}\text { HF-pyr } \\
\text { Purge (t4) }\end{array}$ \\
\hline e & $1-2$ & 1 & 20 & 2 & 15 \\
e & $3-4$ & 0.5 & 20 & 2 & 15 \\
e & $5-6$ & 2 & 20 & 2 & 15 \\
e & $7-8$ & 4 & 20 & 2 & 15 \\
e & $9-10$ & 6 & 20 & 2 & 15 \\
e & $11-12$ & 8 & 20 & 2 & 15 \\
e & $13-14$ & 10 & 20 & 2 & 15 \\
e & $15-20$ & 3 & 20 & 2 & 15 \\
\hline f & $1-4$ & 3 & 20 & 2 & 15 \\
f & $5-8$ & 3 & 20 & 0.5 & 15 \\
f & $9-10$ & 3 & 20 & 1 & 15 \\
f & $11-12$ & 3 & 20 & 3 & 15 \\
f & $13-14$ & 3 & 20 & 4 & 15 \\
f & 15 & 3 & 20 & 5 & 15 \\
f & 16 & 3 & 20 & 2 & 15 \\
& & & & & \\
\hline
\end{tabular}




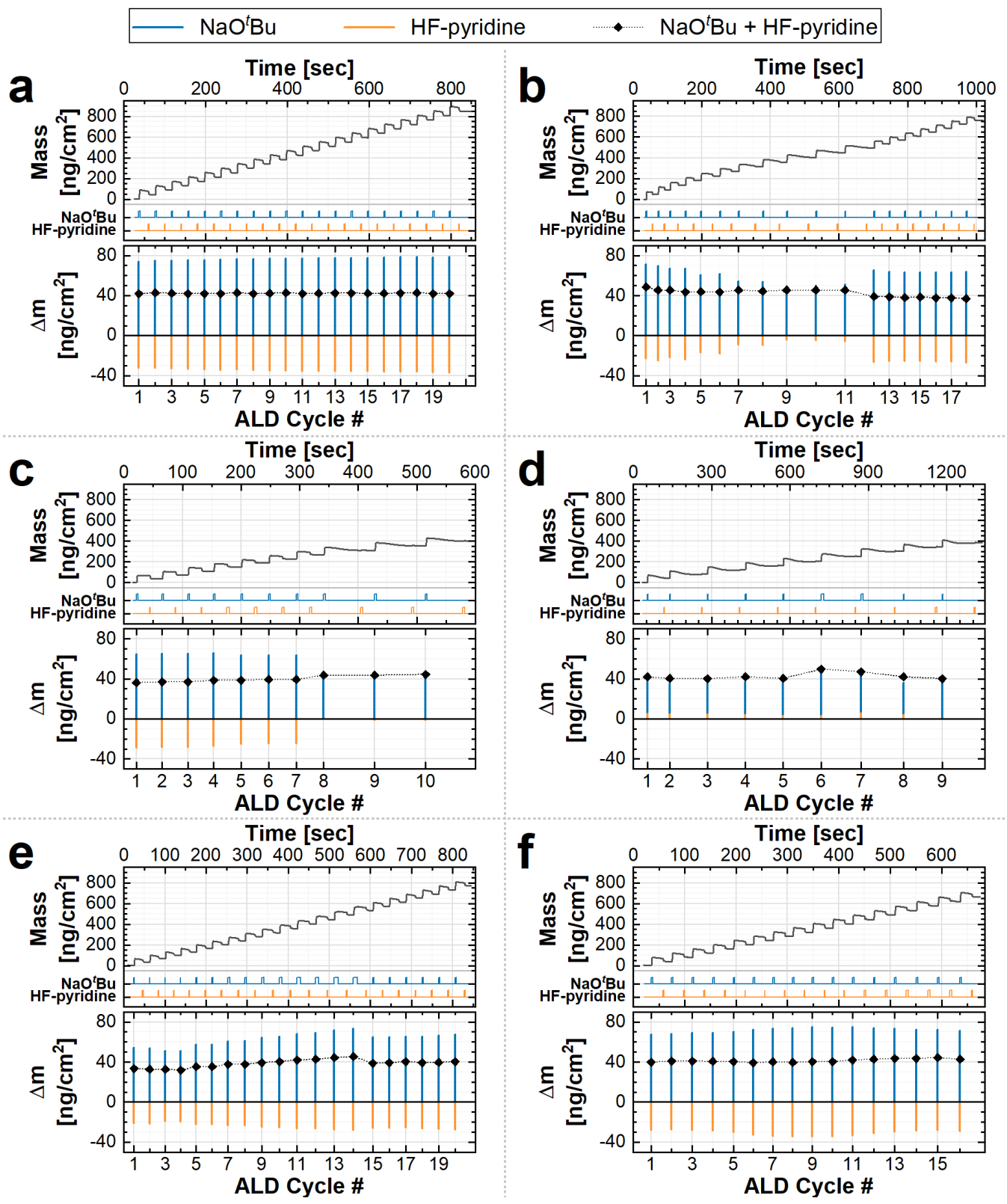

Figure B1 (a) Steady-state NaF growth with 3-20-2-15 cycle timing, reproduced from Fig. 3.2a. (b-f) NaF growth with various precursor dose and purge times provided in Table B1. Net mass change (black diamonds) for complete $\mathrm{NaO}^{t} \mathrm{Bu}+\mathrm{HF}$-pyridine cycle remained near $40-50 \mathrm{ng} / \mathrm{cm}^{2}$ for all dose and purge times shown. The blue and orange bars in the lower traces for each figure represent the mass changes from the individual $\mathrm{NaO}^{t} \mathrm{Bu}$ and $\mathrm{HF}$-pyridine exposures, respectively. The $\mathrm{x}$ - and $\mathrm{y}$-scale values have been shifted so that both start at zero for the graphed data, though additional NaF cycles were completed prior those shown (in the case of Fig. B1a, 39 cycles were completed prior to the steady-state cycles shown). All QCM measurements were performed at $200^{\circ} \mathrm{C}$ and normalized to QCM of alumina (trimethylaluminum + water) to correct for any back-side deposition on the QCM crystal. 


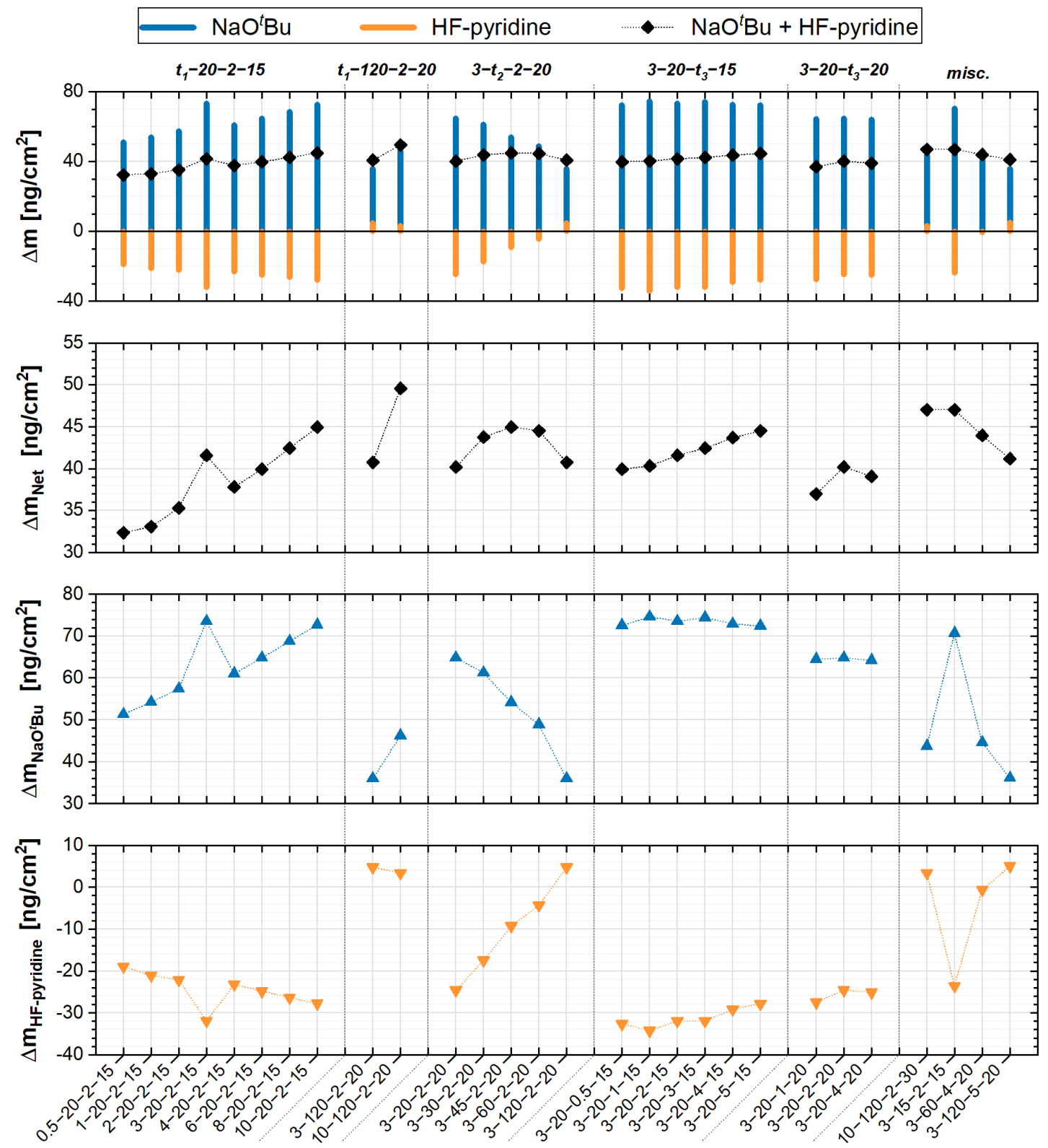

ALD Cycle Timing $\left(t_{1}-t_{2}-t_{3}-t_{4}\right)$ [sec]

Figure B2 Average mass change values for various cycle timing combinations. Similar dosing parameters are grouped together along the horizontal axis. 

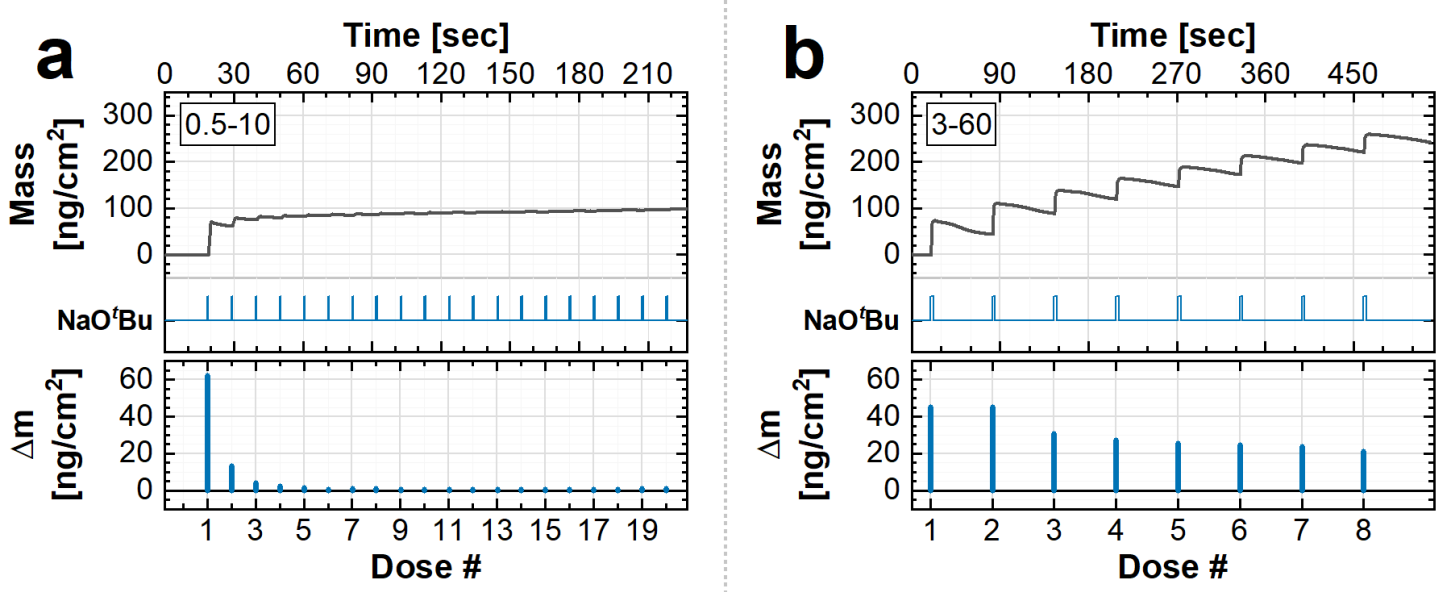

Figure $\mathrm{B3}$ (a) Repeated $\mathrm{NaO}^{t} \mathrm{Bu}$ doses with 0.5-10 half-cycle timing. (b) Repeated $\mathrm{NaO}^{t} \mathrm{Bu}$ dosing with 3-60 half-cycle timing. Under the conditions shown in (b), the surface reactions do not self-limit and subsequent doses are nonsaturating. All QCM measurements were performed at $200{ }^{\circ} \mathrm{C}$ and normalized to QCM of alumina (trimethylaluminum + water) to correct for any back-side deposition on the QCM crystal.

\section{B.2 XPS for Varied Dosing Parameters and Deposition Temperatures}

XPS surface scans for NaF films deposited with 3-20-2-15s dose timing at 175 ${ }^{\circ} \mathrm{C}, 200{ }^{\circ} \mathrm{C}, 225^{\circ} \mathrm{C}$, and $250{ }^{\circ} \mathrm{C}$ are shown in Figures B4-B7, respectively. XPS surface scans for $\mathrm{NaF}$ films deposited at $200{ }^{\circ} \mathrm{C}$ with dose timing of 5-30-1-30s and 3-60-215s are shown in Figures B8 and B9, respectively.

XPS spectra were consistent with $\mathrm{NaF}$ and showed very little oxygen or carbon content, as well as incidental $\mathrm{Ti}$ and $\mathrm{Cl}$ contamination attributed to interactions with previously deposited materials on the reactor walls.

Si 2 s and Si $2 p$ peaks were observed in the survey spectra for all samples, indicating inclusion of signals from the underlying substrate. The relative intensity of $\mathrm{Si}$ peaks was markedly higher for films deposited at $250^{\circ} \mathrm{C}$ (Fig. B7a, inset), which was attributed to film porosity (Figs. 3.6d, B16d, B17d). 
Table B2 XPS Peak fitting results for NaF deposited with 3-20-2-15s cycle timing at various growth temperatures.

\begin{tabular}{|c|c|c|c|c|c|}
\hline \multirow{2}{*}{$\begin{array}{c}\text { ALD } \\
\text { Temp. }\left({ }^{\circ} \mathrm{C}\right)\end{array}$} & \multicolumn{2}{|c|}{ - Na ( RSF = 10.588) - } & \multicolumn{2}{|c|}{-- F ( RSF = 4.118) - } & \multirow{2}{*}{ Na:F } \\
\hline & $\begin{array}{c}\text { Peak Area (raw) } \\
{[c p s \cdot e V]}\end{array}$ & $\begin{array}{c}\text { Peak Area (norm.) } \\
{[\mathrm{cps} \cdot \mathrm{eV}]}\end{array}$ & $\begin{array}{c}\text { Peak Area (raw) } \\
{[\text { cps } \cdot \mathrm{eV}]}\end{array}$ & $\begin{array}{c}\text { Peak Area (norm.) } \\
{[\mathrm{cps} \cdot \mathrm{eV}]}\end{array}$ & \\
\hline 175 & 1389245.55 & 3996.68 & 910337.27 & 4209.46 & $1:: 1.05$ \\
\hline 200 & 1380370.14 & 3970.74 & 900301.34 & 4162.65 & $1: 1.05$ \\
\hline 225 & 1392057.08 & 4002.82 & 885490.92 & 4093.20 & $1: 1.02$ \\
\hline 250 & 1192371.77 & 3427.50 & 722147.30 & 3337.42 & $1: 0.97$ \\
\hline
\end{tabular}

* RSF values from Thermo Scientific Avantage XPS software. 

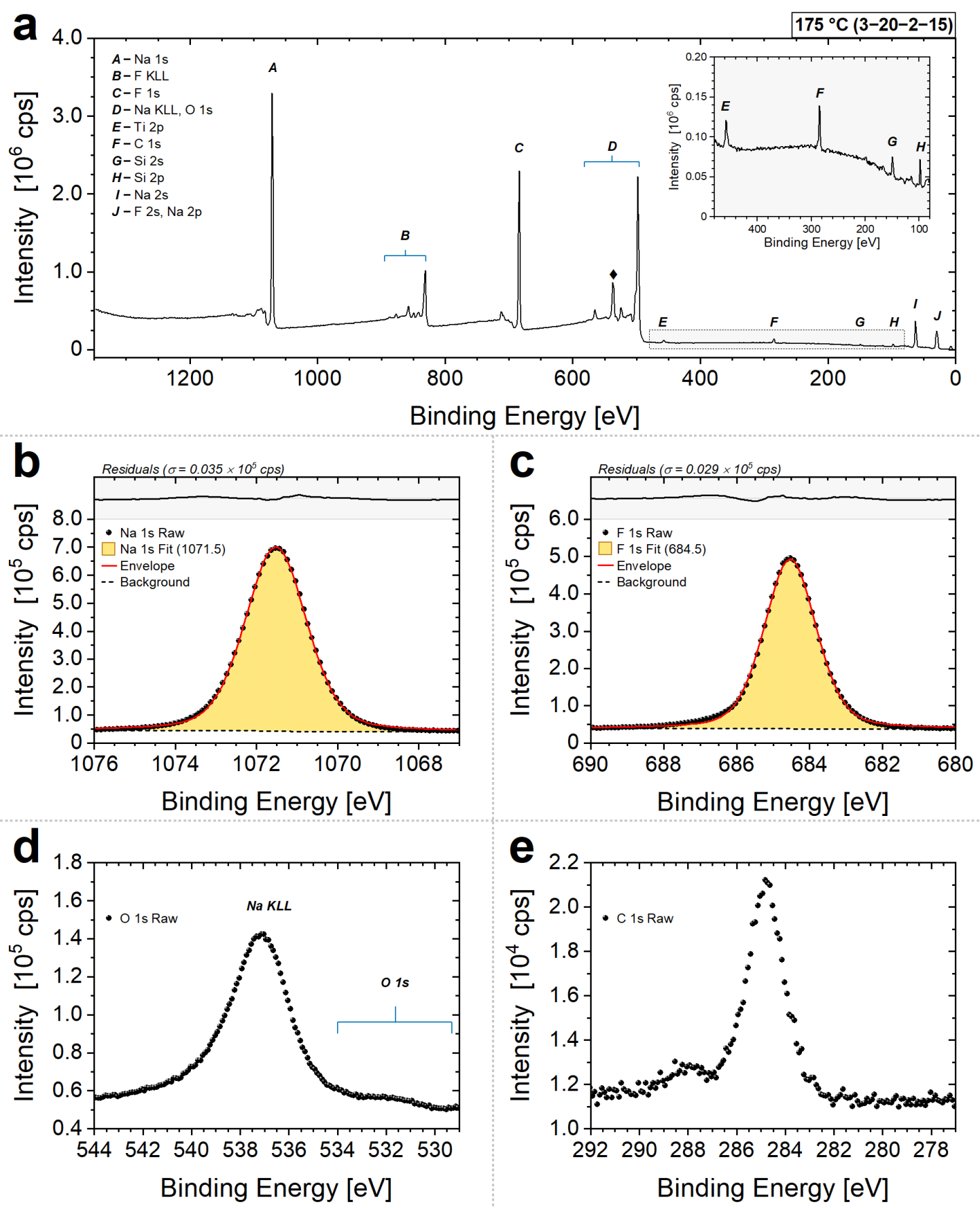

Figure B4 XPS surface spectra for NaF film deposited at $175^{\circ} \mathrm{C}$ with 3-60-215 cycle timing. Films were grown on silicon substrates with native oxide. (a) Survey scan, (b) Sodium 1s region, (c) Fluorine 1s region, (d) Oxygen 1s region with overlap of sodium KLL Auger peaks, (e) Carbon 1s region. For reference, the prominent Na KLL peak from panel (d) is marked with a black diamond in panel (a). 

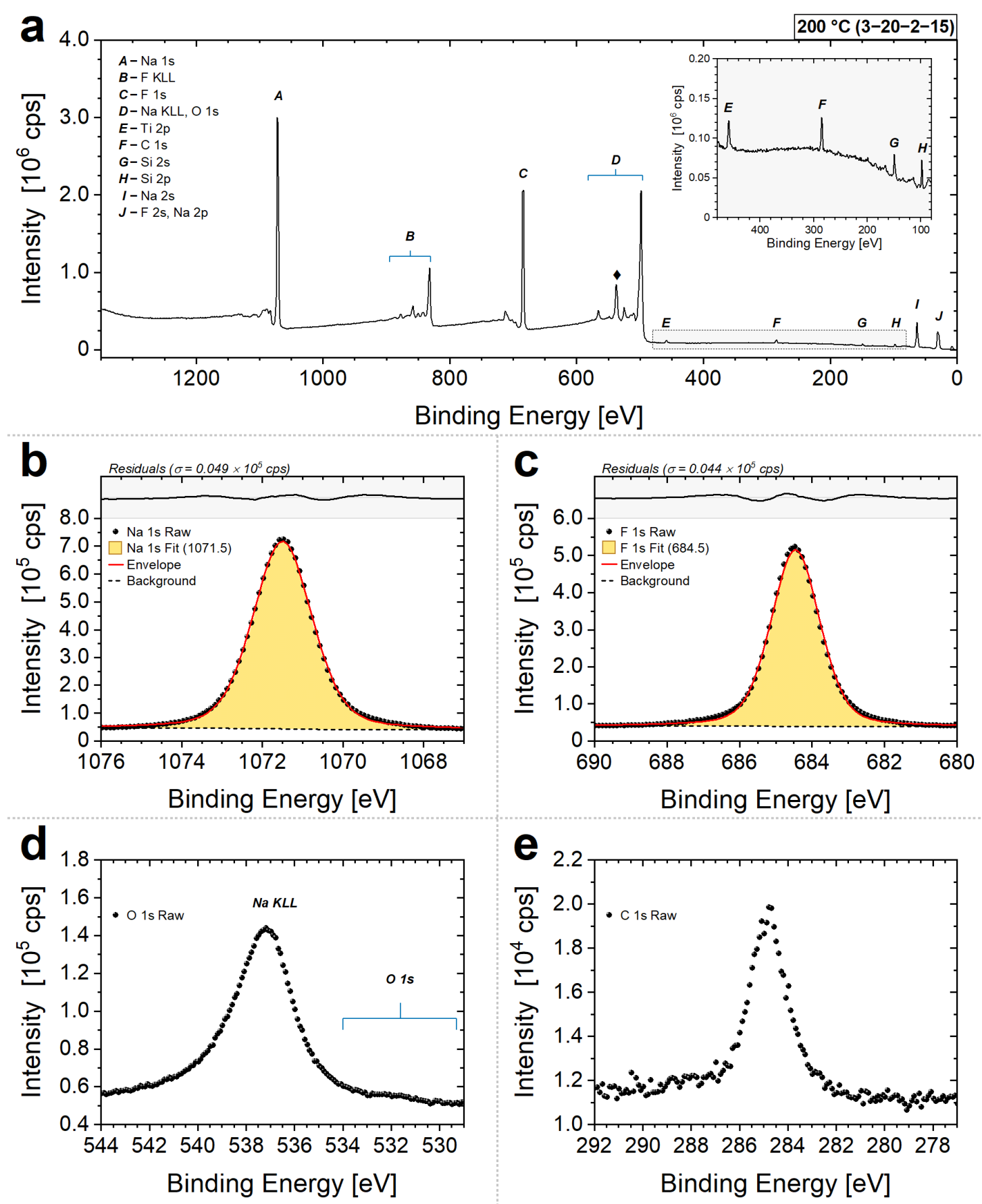

Figure B5 XPS surface spectra for NaF film deposited at $200{ }^{\circ} \mathrm{C}$ with 3-20-215 cycle timing. Films were grown on silicon substrates with native oxide. (a) Survey scan, (b) Sodium 1s region, (c) Fluorine 1s region, (d) Oxygen 1s region with overlap of sodium KLL Auger peaks, (e) Carbon 1s region. For reference, the prominent Na KLL peak from panel (d) is marked with a black diamond in panel (a). Panels a-c reproduced from Fig. 3.3. 

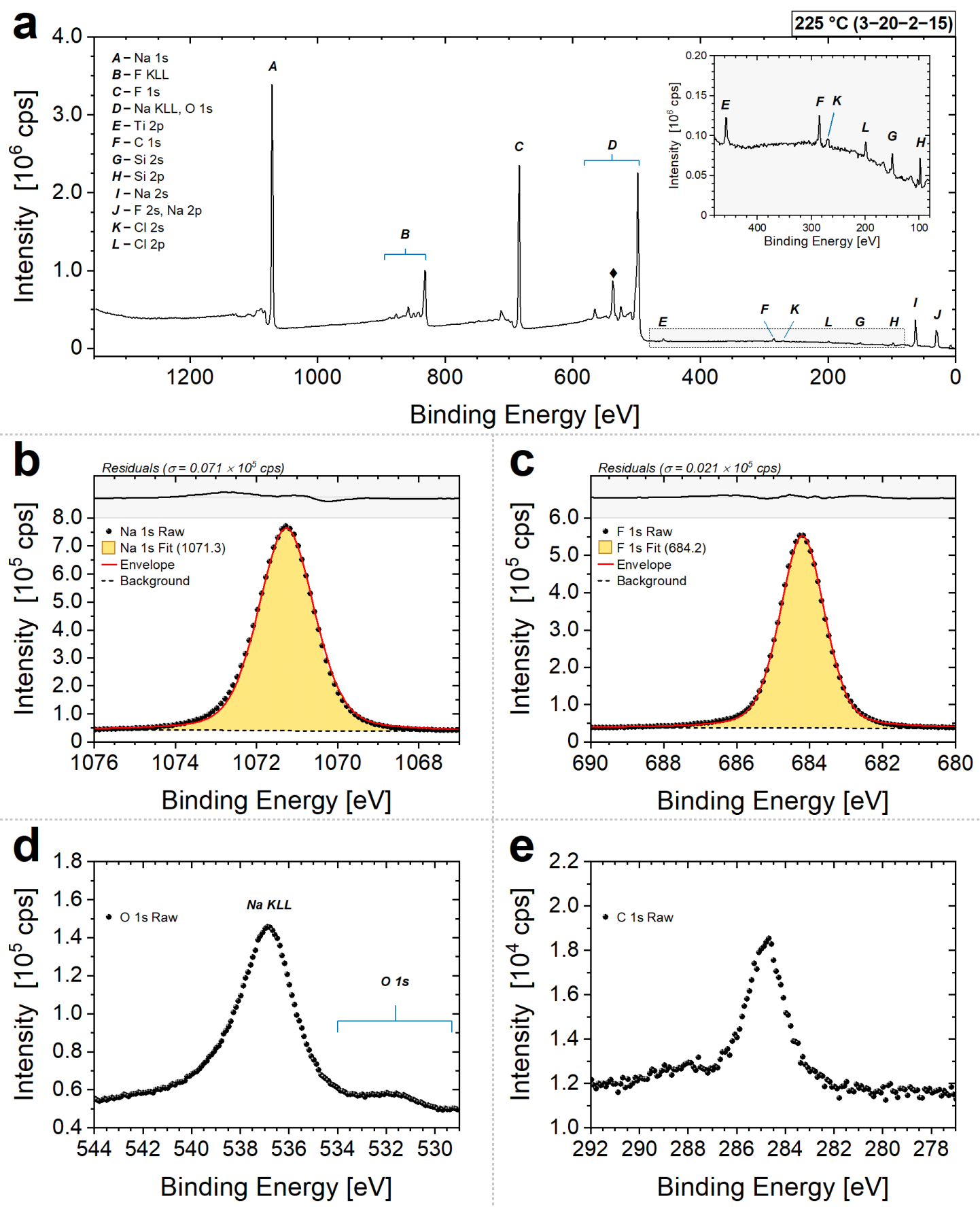

Figure B6 XPS surface spectra for NaF film deposited at $225^{\circ} \mathrm{C}$ with 3-60-215 cycle timing. Films were grown on silicon substrates with native oxide. (a) Survey scan, (b) Sodium 1s region, (c) Fluorine 1s region, (d) Oxygen 1s region with overlap of sodium KLL Auger peaks, (e) Carbon 1s region. For reference, the prominent Na KLL peak from panel (d) is marked with a black diamond in panel (a). 

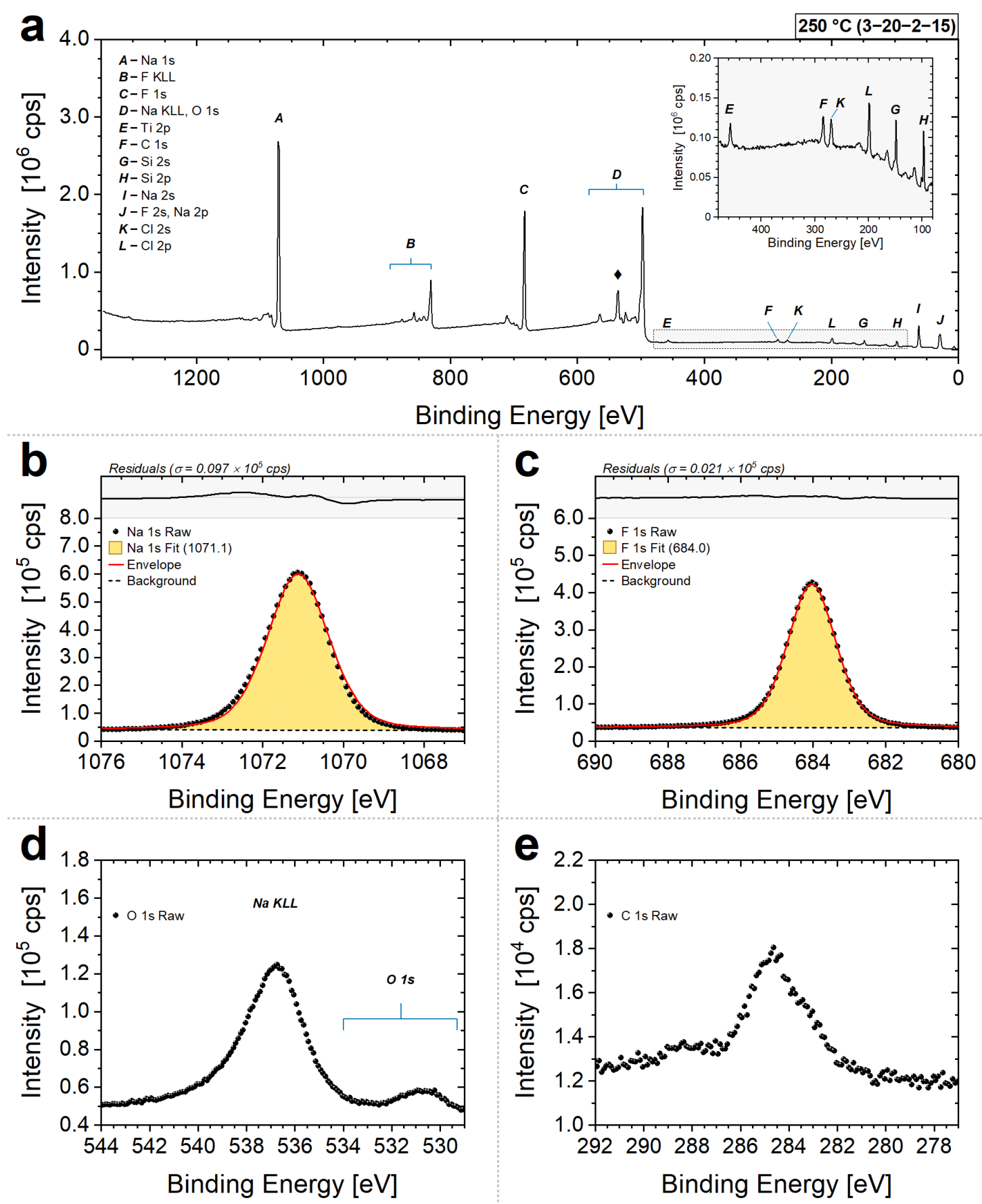

Figure B7 XPS surface spectra for NaF film deposited at $250{ }^{\circ} \mathrm{C}$ with 3-60-215 cycle timing. Films were grown on silicon substrates with native oxide. (a) Survey scan, (b) Sodium 1s region, (c) Fluorine 1s region, (d) Oxygen 1s region with overlap of sodium KLL Auger peaks, (e) Carbon 1s region. For reference, the prominent Na KLL peak from panel (d) is marked with a black diamond in panel (a). 

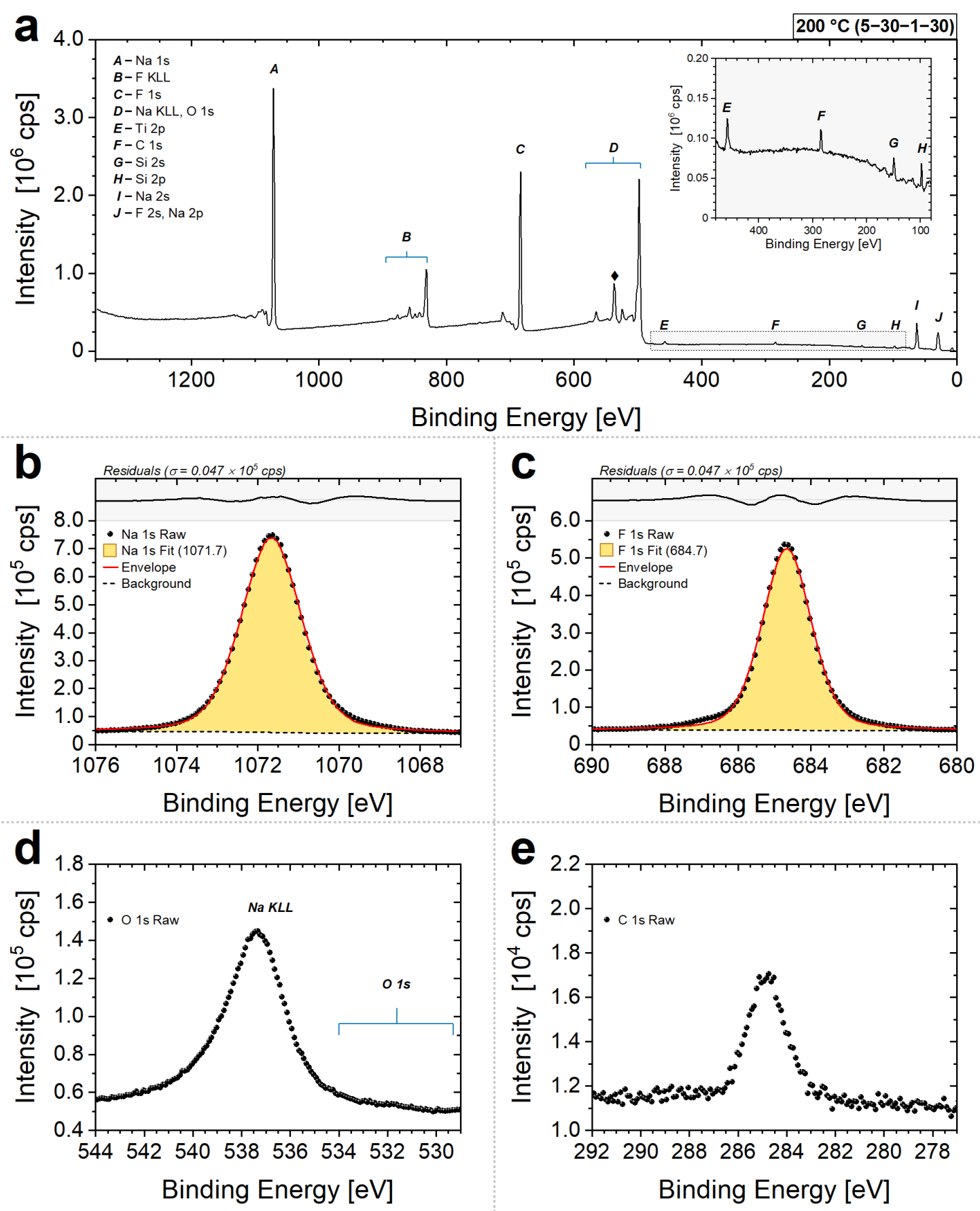

Figure B8 XPS surface spectra for NaF film deposited at $200{ }^{\circ} \mathrm{C}$ with 5-30-130 cycle timing. Films were grown on silicon substrates with native oxide. (a) Survey scan, (b) Sodium 1s region, (c) Fluorine 1s region, (d) Oxygen 1s region with overlap of sodium KLL Auger peaks, (e) Carbon 1s region. For reference, the prominent Na KLL peak from panel (d) is marked with a black diamond in panel (a). 

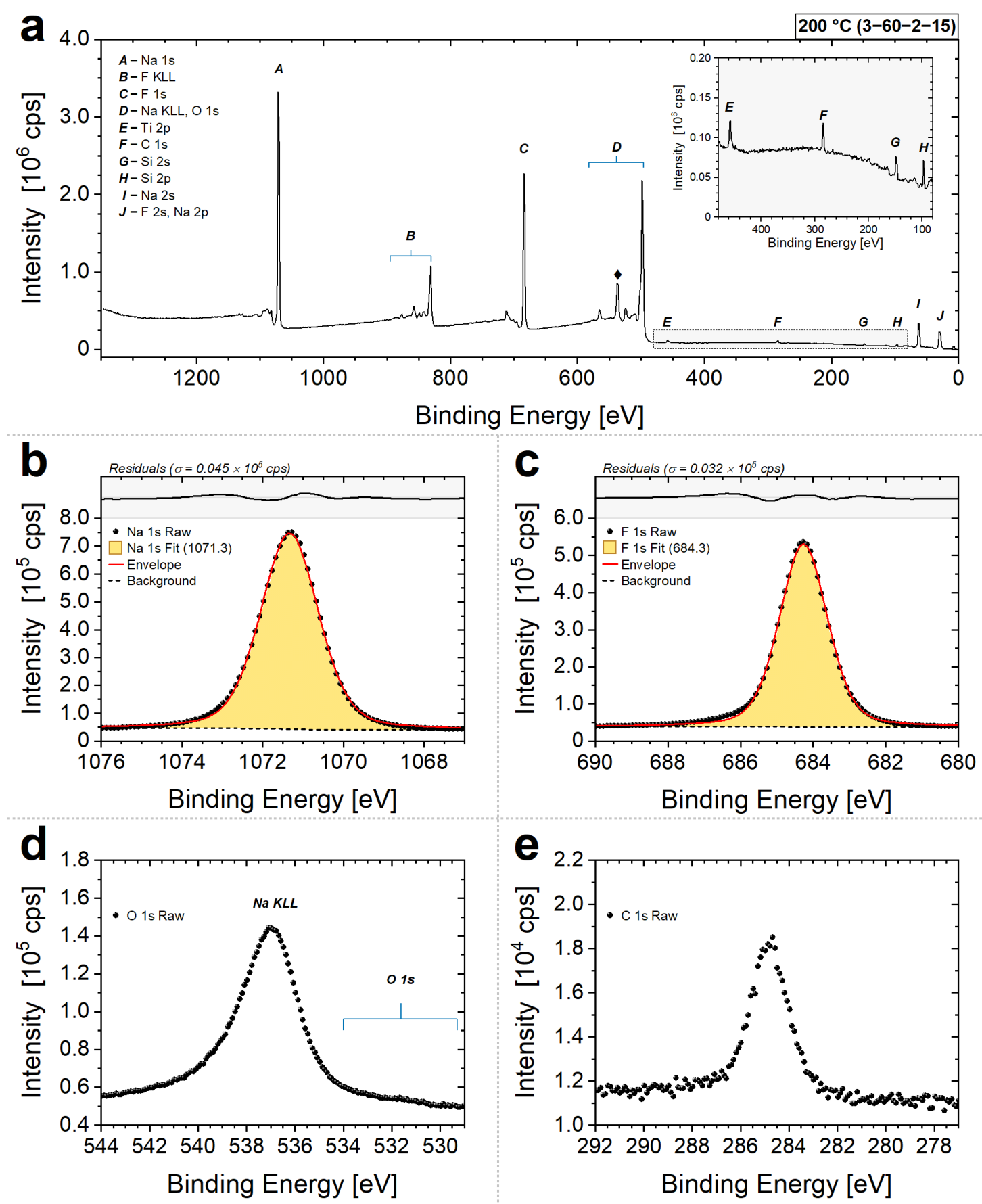

Figure B9 XPS surface spectra for NaF film deposited at $200{ }^{\circ} \mathrm{C}$ with 3-60-215 cycle timing. Films were grown on silicon substrates with native oxide. (a) Survey scan, (b) Sodium 1s region, (c) Fluorine 1s region, (d) Oxygen 1s region with overlap of sodium KLL Auger peaks, (e) Carbon 1s region. For reference, the prominent Na KLL peak from panel (d) is marked with a black diamond in panel (a). 


\section{B.3 Spectroscopic Ellipsometry - NaF Sellmeier Model}

Measured spectroscopic ellipsometry (SE) data for $\mathrm{NaF}$ deposition was fit using the Sellmeier equation, which treats atoms in a material as elastic oscillators subject to a periodic force due to interactions with incident light waves. Unlike the Cauchy equation, which is an empirical formula that is not physically meaningful, the Sellmeier equation is consistent with more rigorous dispersion derivations based on electromagnetic theoryso long as the fitting region does not significantly overlap with the material's absorption bands. The Sellmeier equation is applicable across much larger spectral ranges than the Cauchy equation, and it is able to approximate so-called "anomalous" dispersion on either side of an absorption band where the Cauchy equation breaks down. ${ }^{1}$ The Sellmeier model is routinely used for fitting the optical constants of transparent dielectric films, and its 2-term form used for fitting in the CompleteEASE software is given by Equation B1. ${ }^{2}$

$$
n=\left(\varepsilon(\infty)+\frac{A \lambda^{2}}{\lambda^{2}-B^{2}}-E \lambda^{2}\right)^{\frac{1}{2}}
$$

The index of refraction, $n$, is calculated for wavelength $\lambda$ using the Sellmeier parameters $\varepsilon(\infty)$ (index offset), $A$ (amplitude), $B$ (center energy), and $E$ (position of a pole in the infrared).

$\mathrm{NaF}$ films grown on Si were fit using two model layers on a Si substrate as shown in Figure B10, while three model layers were used for $\mathrm{NaF}$ deposition on $\mathrm{Al}_{2} \mathrm{O}_{3}$ coated $\mathrm{Si}$, as shown in Figure B11. NaF films for SE modelling were deposited via 100 ALD cycles of $\mathrm{NaO}^{t} \mathrm{Bu}+\mathrm{HF}-$ pyridine with $3-20-2-15$ s cycle timing.

Ellipsometric data and fitting results are provided in Figures B12 and B13 for NaF films deposited with 3-20-2-15s dose timing at $175^{\circ} \mathrm{C}, 200{ }^{\circ} \mathrm{C}, 225^{\circ} \mathrm{C}$, and 250 
${ }^{\circ} \mathrm{C}$. Additional SE data and fitting results for $\mathrm{NaF}$ films deposited at $200{ }^{\circ} \mathrm{C}$ with dose timing of 5-30-1-30s and 3-60-2-15s are shown in Figure B14.

Although all SE fits yielded mean squared error (MSE) values less than 2, the $\mathrm{NaF}$ Sellmeier model deviated from measured Delta data at higher wavelengths for 250 ${ }^{\circ} \mathrm{C}$ samples (see Supplemental Information Fig. B13c-d). This discrepancy may be due to film non-uniformity (Fig. 3.6d, Supplemental Information Figs. B16d and B17d) and/or incidental chlorine contamination (Supplemental Information Fig. B7a) at higher growth temperatures.

Ellipsometric data for $\mathrm{NaF}$ deposited at $200{ }^{\circ} \mathrm{C}$ on $\mathrm{Al}_{2} \mathrm{O}_{3}$ coated $\mathrm{Si}$ is shown in Figure B15. The data are well fit by the model and support a larger GPC for growth on $\mathrm{Al}_{2} \mathrm{O}_{3}$, relative to $\mathrm{Si}$, consistent with the in situ QCM observations.

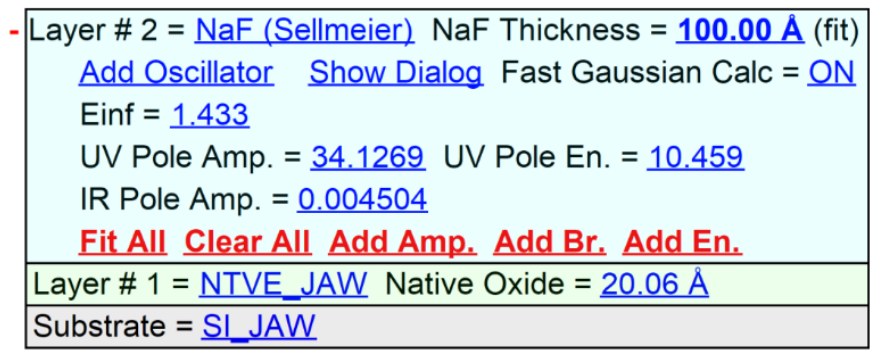

Figure B10 CompleteEASE layers used to model NaF film on Si. Substrate = silicon ("SI_JAW"), Layer \#1 = native oxide ("NTVE_JAW"), Layer \#2 = NaF ("NaF (Sellmeier)"). The Layer \#2 Sellmeier parameters "Einf," "UV Pole Amp.," "UV Pole En.," and "IR Pole Amp." correspond to Equation B1 variables $\varepsilon(\infty), A, B$, and $E$, respectively. 


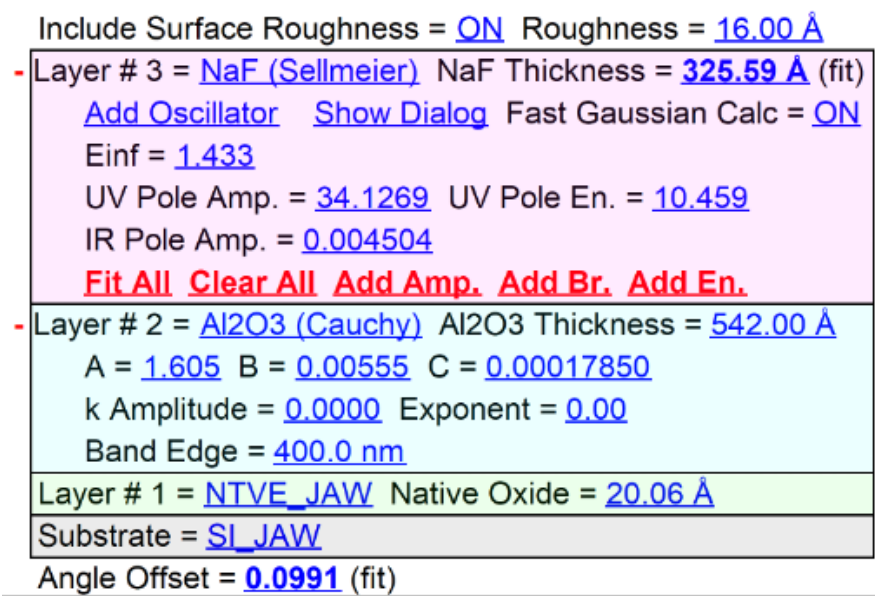

Figure B11 CompleteEASE layers used to model NaF film deposited at $200{ }^{\circ} \mathrm{C}$ on $\mathrm{Al}_{2} \mathrm{O}_{3}$ coated $\mathrm{Si}$. Substrate = silicon ("SI_JAW"), Layer \#1 = native oxide ("NTVE_JAW"), Layer \#2 = ALD $\mathrm{Al}_{2} \mathrm{O}_{3}$, Layer \#3 = NaF ("NaF (Sellmeier)"). The surface roughness value measured from AFM was included. The Layer \#3 Sellmeier parameters "Einf," "UV Pole Amp.," "UV Pole En.," and "IR Pole Amp." correspond to Equation B1 variables $\varepsilon(\infty), A, B$, and $E$, respectively. 

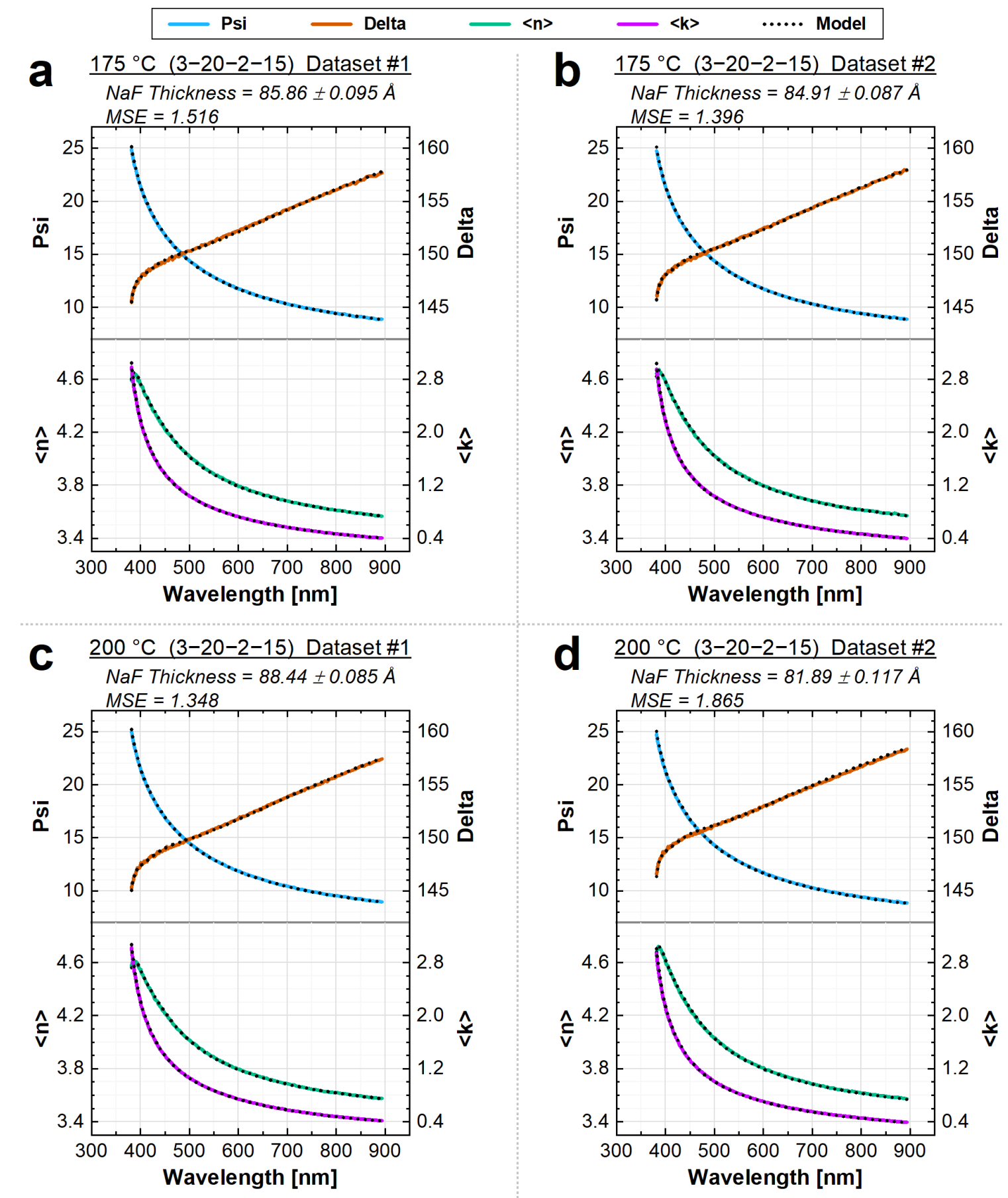

Figure B12 SE data and modelling results for NaF films deposited via 100 ALD cycles of $\mathrm{NaO}^{t} \mathrm{Bu}+\mathrm{HF}$-pyridine at (a-b) $175^{\circ} \mathrm{C}$ and (c-d) $200{ }^{\circ} \mathrm{C}$ with 3-20-2-15 cycle timing. Duplicate samples for each temperature were taken from opposite ends of the reactor chamber (separated by $\sim 50 \mathrm{~cm}$ ). 

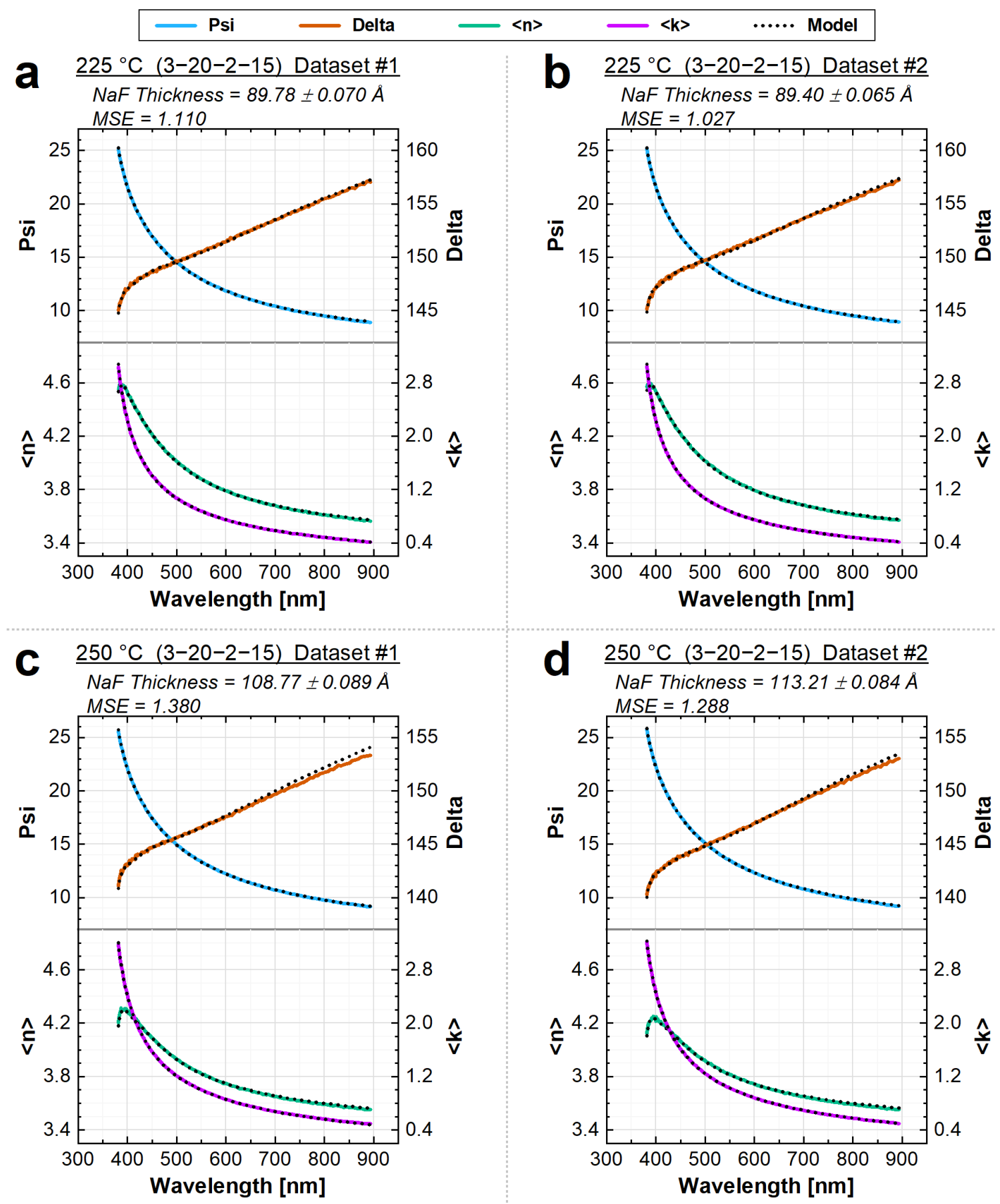

Figure B13 SE data and modelling results for NaF films deposited via 100 ALD cycles of $\mathrm{NaO}^{t} \mathrm{Bu}+\mathrm{HF}$-pyridine at (a-b) $225^{\circ} \mathrm{C}$ and (c-d) $250{ }^{\circ} \mathrm{C}$ with 3-20-2-15 cycle timing. Duplicate samples for each temperature were taken from opposite ends of the reactor chamber (separated by $\sim 50 \mathrm{~cm}$ ). 

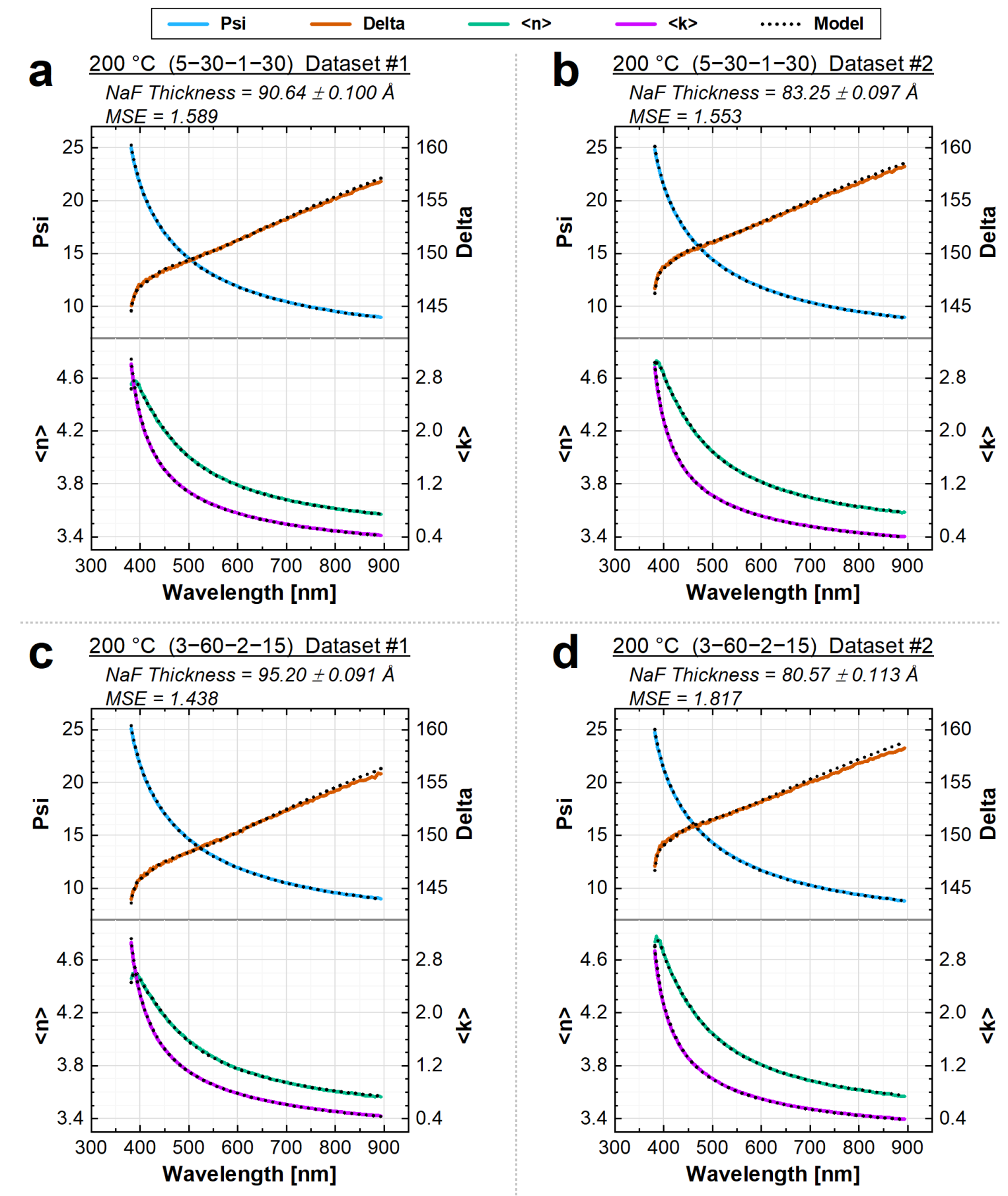

Figure B14 SE data and modelling results for NaF films deposited via 100 ALD cycles of $\mathrm{NaO}^{t} \mathrm{Bu}+\mathrm{HF}$-pyridine at $200{ }^{\circ} \mathrm{C}$ with (a-b) 5-30-1-30 and (c-d) 3-60-215 cycle timing. Duplicate samples for each temperature were taken from opposite ends of the reactor chamber (separated by $\sim 50 \mathrm{~cm})$. 


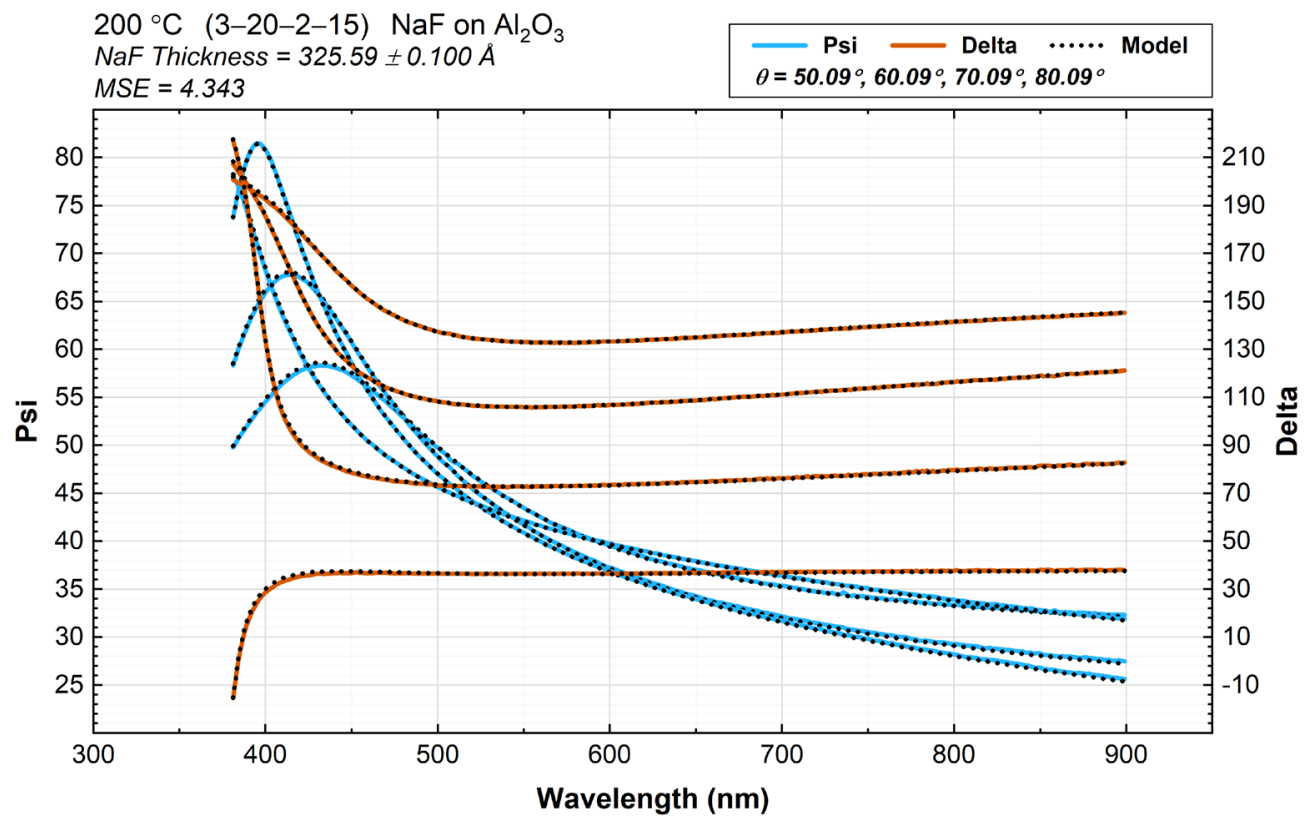

Figure $\mathrm{B} 15 \mathrm{SE}$ data and modelling results for $\mathrm{NaF}$ films deposited on $\mathrm{Al}_{2} \mathrm{O}_{3}$ coated silicon via $100 \mathrm{ALD}$ cycles of $\mathrm{NaO}^{t} \mathrm{Bu}+\mathrm{HF}$-pyridine at $200{ }^{\circ} \mathrm{C}$ with $3-20-2$ 15 cycle timing. 


\section{B.4 NaF Surface Morphology - Ambient AFM of Air-Exposed NaF Samples}

Images of air-exposed $\mathrm{NaF}$ surfaces were obtained in ambient conditions with a Bruker Dimension FastScan operating in peak-force tapping mode using ScanAsyst-AirHR probes. Samples were removed from the Ar storage environment and imaged in ambient conditions after $\sim 30$ min of air exposure. Surface roughness $\mathrm{R}_{\mathrm{q}}$ values for films deposited at $175{ }^{\circ} \mathrm{C}, 200{ }^{\circ} \mathrm{C}, 225^{\circ} \mathrm{C}$, and $250{ }^{\circ} \mathrm{C}$ were $\sim 1.6 \mathrm{~nm}, \sim 1.7 \mathrm{~nm}, \sim 2.5 \mathrm{~nm}$, and $\sim 6.4 \mathrm{~nm}$, respectively.
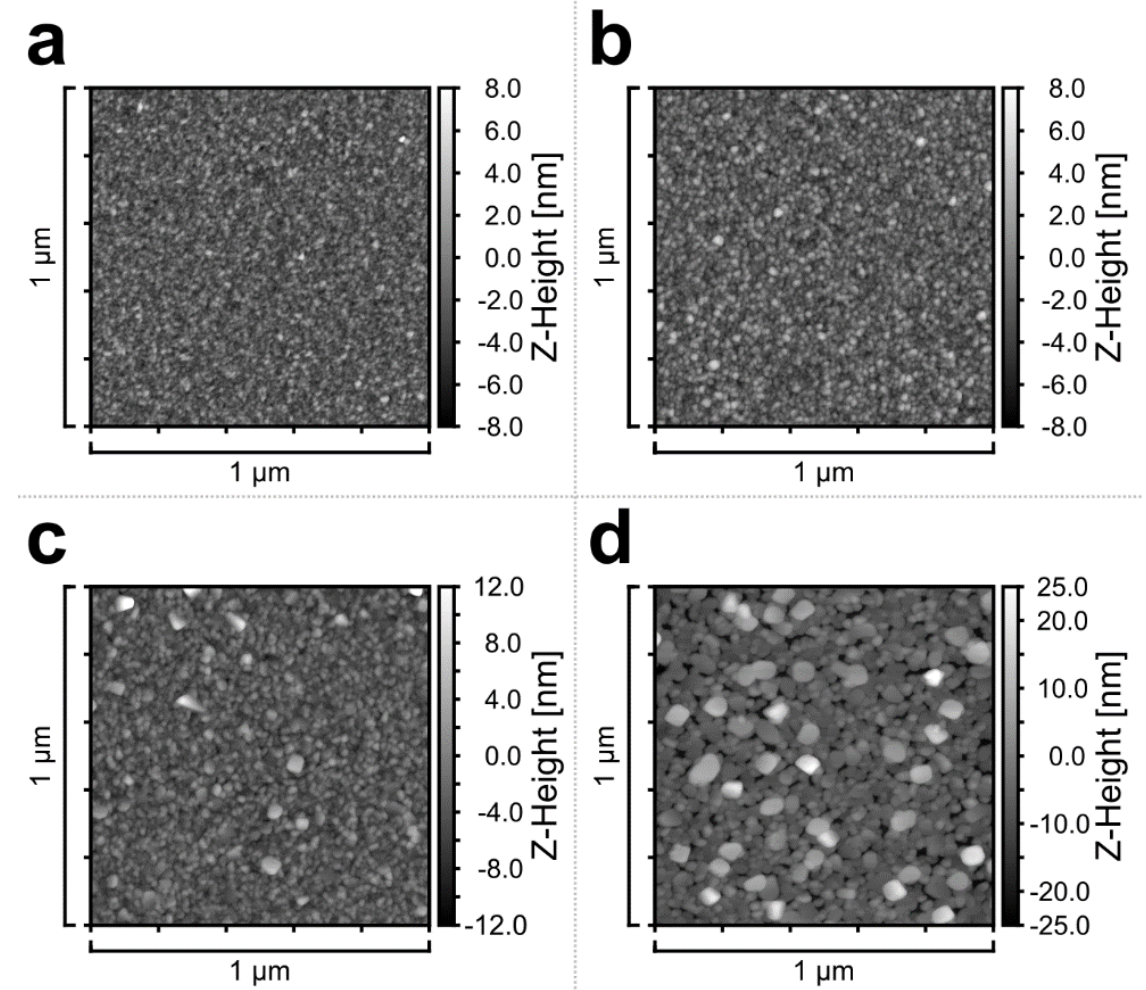

Figure B16 AFM images of NaF surface morphology for air-exposed films deposited on silicon substrates at growth temperatures of (a) $175^{\circ} \mathrm{C}$, (b) $200{ }^{\circ} \mathrm{C}$, (c) $225^{\circ} \mathrm{C}$, and (d) $250{ }^{\circ} \mathrm{C}$. 
B.5 NaF Surface Morphology - Scanning Electron Microscopy of Air-Exposed NaF

\section{Samples}
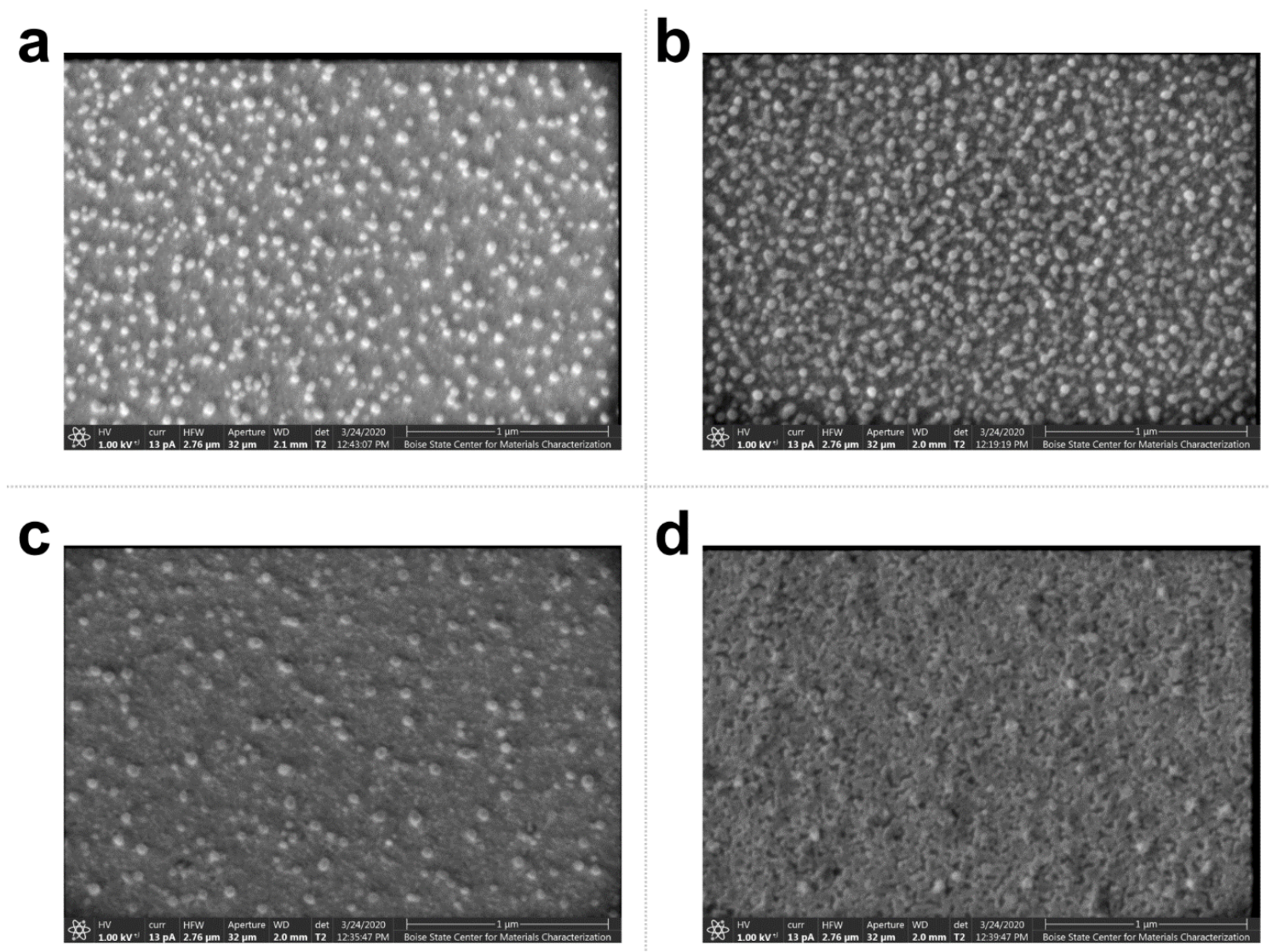

Figure B17 SEM micrographs of NaF surface morphology for air-exposed films deposited on silicon substrates at growth temperatures of (a) $175^{\circ} \mathrm{C}$, (b) $200^{\circ} \mathrm{C}$, (c) $225^{\circ} \mathrm{C}$, and (d) $250^{\circ} \mathrm{C}$.

\section{B.6 References}

1. Jenkins, F. A.; White, H. E., Dispersion. In Fundamentals of Optics, 3rd ed.; McGraw-Hill: 1957.

2. J.A. Woolam Co., Inc. (2014). CompleteEASE: Software Manual. Lincoln, NE: J.A. Woolam Co, Inc. 